Guilherme Wesley Peixoto da Fonseca

Efeitos da deficiência anabólica hormonal e alterações neurovasculares no prognóstico de pacientes com insuficiência cardíaca

Tese apresentada à Faculdade de Medicina da Universidade de São Paulo para obtenção do título de Doutor em Ciências

Programa de Cardiologia

Orientadora: Profa. Dra. Maria Janieire de Nazaré Nunes Alves

São Paulo 
Guilherme Wesley Peixoto da Fonseca

Efeitos da deficiência anabólica hormonal e alterações neurovasculares no prognóstico de pacientes com insuficiência cardíaca

Tese apresentada à Faculdade de Medicina da Universidade de São Paulo para obtenção do título de Doutor em Ciências

Programa de Cardiologia

Orientadora: Profa. Dra. Maria Janieire de Nazaré Nunes Alves

São Paulo 


\section{Dados Internacionais de Catalogação na Publicação (CIP)}

Preparada pela Biblioteca da

Faculdade de Medicina da Universidade de Săo Paulo

Creproduçăo autorizada pelo autor

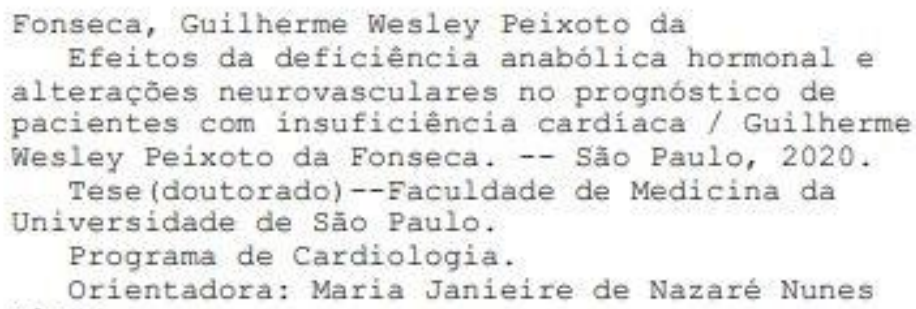

Descritores: 1.Prognóstico 2. Insuficiência cardíaca 3.Testosterona 4.Sarcopenia 5.Obesidade sarcopênica 6 . Desbalanço autonômico 7. Pletismografía 8. Doença de Chagas

$\mathrm{USP} / \mathrm{FM} / \mathrm{DBD}-210 / 20$

Responsável: Erinalva da Conceição Batista, CRB-8 6755 


\section{Dedicatória}

Dedico este trabalho aos meus pais, Ana Claudia Martins Peixoto da Silva e Carlos Roberto da Silva, e a minha irmã Kethellin Peixoto da Silva. Obrigado por compartilharem essa jornada comigo, sempre com muito amor e carinho em momentos decisivos da minha formação acadêmica. O apoio de vocês foi fundamental para alcançar esse sonho. Amo muito vocês! 


\section{Agradecimentos}

Primeiramente, agradeço a Deus por me dar motivação, direcionamento e força em todos os dias dessa caminhada. Agradeço a toda minha família pelo incentivo, em especial ao meu avô (in memoriam) por ser um exemplo de perseverança e consistência. A minha noiva Verônica (minha dádiva) por extrair sempre o melhor de mim e me encorajar nessa jornada. Agradeço também à família da minha noiva por todo apoio (Rosana, Matheus, Natália), inclusive técnico com as dúvidas sobre estatística sanadas com meu sogro Evandro.

A todos os voluntários que participaram deste estudo. A cooperação e disponibilidade dos pacientes e familiares foram de grande valia para o desenvolvimento deste trabalho.

A minha orientadora, Doutora Maria Janieire de Nazaré Nunes Alves (Dra Jane), por ter me aberto esta porta em um momento crucial da minha carreira acadêmica. Obrigado pela confiança depositada em mim e todo aprendizado. Graças a oportunidade concedida, tive o privilégio de ir muito além do que tinha planejado para o doutorado, principalmente quando tinha desistido de realizar o doutorado sanduíche por diversos motivos. Agradeço de coração!

Ao meu grande amigo Marcelo Rodrigues dos Santos, sempre paciente e disposto a me "coorientar". Seus ensinamentos e insights foram de extrema importância para o meu desenvolvimento profissional. Você e sua esposa, Thaís, são meus "pais" acadêmicos, que já me ajudam desde a graduação. Ao meu grande amigo Francis Ribeiro de Souza (Frank, “tá certo!?”) pela ajuda na força tarefa na realização de todos os exames de microneurografia e plestismografia. Agradeço também ao companheirismo nas bandejadas e risadas. Ao meu amigo Rafael Armani (Coach, "qual 
o protocolo hoje?"), por toda a ajuda na execução do projeto e aos almoços com bife à parmegiana no boteco acompanhados de café com chocolate. A colaboração e a parceria de vocês foram primordiais na execução do projeto e no alívio, de uma forma bem humorada, do estresse gerado pelo doutorado. Vocês são feras ferozes! Agradeço de coração!

Aos demais amigos do grupo, Kelly Correia, Caio Novaes, Brenda e Antonio Battaglia, pela ajuda nos exames, estudos e por estarem ao meu lado nestes anos.

Aos coautores dos artigos publicados, pela parceria e auxílio na execução do projeto. Agradeço também ao $\operatorname{Prof}^{\circ}$ Stefan von Haehling e equipe (Anja, Tânia, Nicole, Laura e Mirela) pela recepção em Göttingen, pelos ensinamentos acadêmicos e pela oportunidade de participar de um dos principais grupos de estudo sobre sarcopenia/caquexia na atualidade, contribuindo imensamente para o meu desenvolvimento científico internacional.

À Prof ${ }^{a}$ Dra. Ivani Credidio Trombetta pela recepção na Unidade de Reabilitação Cardiovascular e Fisiologia do Exercício do Instituto do Coração (InCor) no início do meu estágio em 2011. Agradeço por toda orientação e aprendizado transmitido durante esses anos. Ao Prof ${ }^{\circ}$ Eduardo Rondon (Pai Edu) por todo aprendizado prático durante essa fase de estágio na Escola de Educação Física e Esporte da USP (EEFE-USP). Agradeço também aos Profissionais de Educação Física da Unidade, Prof ${ }^{\circ}$ Newton Nunes (conheci o programa de reabilitação do InCor em uma de suas aulas de pósgraduação ministrada na Universidade Gama Filho), Prof ${ }^{a}$ Camila Paixão Jordão (Cah), Prof $^{a}$ Luciana Santos (Lu), Prof ${ }^{a}$ Daniela Agostinho (Dani), Prof $^{a}$ Ana Claudia, Prof ${ }^{o}$ Renato Pelaquim (meu parceiro da época de Dante Pazzanese, onde realizava o estágio no setor de Reabilitação). Vocês são exemplos da importância do Profissional de Educação Física em uma equipe multidisciplinar. 
À equipe médica da Unidade de Reabilitação Cardiovascular e Fisiologia do Exercício do InCor, Dra. Patrícia Oliveira, Dra. Ana Maria Braga e Dra. Fabiana Hodas por todo aprendizado e os encaixes de exames realizados de última hora. Agradecimento especial ao meu amigo, Dr. Marcel Costa, por todos os ensinamentos e colaboração na realização dos exames.

Aos amigos de laboratório, Allan Krusler (obrigado pela receptividade no congresso do Rio), Thiago de Oliveira, Patrícia Trevizan (Paty), Raphaela Groehs (Rapha), Adriana Sarmento (Dri), Luciene Azevedo, Sara Rodrigues (Sarinha), Renan Guerra (Marreta), Larissa dos Santos, Carlos Alcino, Edna Oliveira e Yufuko (Ioko). Aos amigos do "bandejão" Gustavo Souza, Pedro Senger e Bruno Durante, pelo companheirismo e convivência nestes anos (apesar do curso de vocês serem na “modalidade EAD”). Obrigado por todo aprendizado e os momentos de descontração e risadas!

Aos amigos que cursaram comigo a especialização em condicionamento físico aplicado à prevenção cardiológica primária e secundária (Turma 2012), Letícia, André, Heverton, Thiago e Gabriela, pelo encorajamento e companheirismo!

Aos meus amigos e alunos da Academia VO2 personal por todo incentivo no início do doutorado. Agradeço a Prof ${ }^{a}$ Jaqueline Pivello pela confiança depositada no meu trabalho. Em especial, ao meu amigo Prof ${ }^{\circ}$ Átila Vidigal, pelos ensinamentos e paixão demonstrada na aplicação do treinamento físico.

Às secretarias da Unidade de Reabilitação Cardiovascular e Fisiologia do Exercício do InCor, Mônica Marques (Moniquinha), Sandra Sino (Sandroka), Rosângela Aureliano (Rô), Fabiana Pereira (Fabi), Elaine e Patrick pelos momentos de descontração e auxílio em processo burocráticos da instituição. À equipe da secretaria da pós-graduação em cardiologia e comissão científica do InCor, Neuza Rodrigues, 
Juliana Lattari, Luana Guedes, Valdecira Ferreira, Monica Souto, Sidnei Silva, Elaine Lagonegro e Fabiana Panham pela paciência e por todo auxílio institucional durante o doutorado.

Ao Diretor da Unidade de Reabilitação Cardiovascular e Fisiologia do Exercício do InCor, Prof. Dr. Carlos Eduardo Negrão, por abrir as portas do laboratório e pelo grande aprendizado científico.

Ao Conselho Nacional de Desenvolvimento Científico e Tecnológico (CNPq; processo $n^{\circ}$ 148758/2016-9) e a Coordenação de Aperfeiçoamento de Pessoal de Nível Superior (CAPES; processo nº 88887.368499/2019-00) pelo apoio financeiro à pesquisa com as bolsas de doutorado e doutorado sanduíche, respectivamente. A Faculdade de Medicina da Universidade de São Paulo (FMUSP) e ao InCor pela estrutura cedida que contribuiu para o meu aprimoramento científico e acadêmico. 
Epígrafe

“Àquele que é capaz de fazer infinitamente mais do que tudo o que pedimos ou pensamos, de acordo com o seu poder que atua em nós,"

Efésios 3:20 


\section{Normatização adotada}

Esta tese está de acordo com as seguintes normas, em vigor no momento desta publicação:

Apresentação de dissertações e teses sob forma de compilação de artigos, aprovado em 16 de setembro de 2011, pela Comissão de Pós-Graduação da Faculdade de Medicina da Universidade de São Paulo.

Referências: adaptado de International Committee of Medical Journals Editors (Vancouver).

Universidade de São Paulo. Faculdade de Medicina. Divisão de Biblioteca e Documentação. Guia de apresentação de dissertações, teses e monografias. Elaborado por Anneliese Carneiro da Cunha, Maria Julia de A. L. Freddi, Maria F. Crestana, Marinalva de Souza Aragão, Suely Campos Cardoso, Valéria Vilhena. $3^{\mathrm{a}}$ ed. São Paulo: Divisão de Biblioteca e Documentação; 2011.

Abreviaturas dos títulos dos periódicos de acordo com List of Journals Indexed in Index Medicus. 


\section{SUMÁRIO}

\section{Dedicatória}

Agradecimentos

Epígrafe

Normatização adotada

Resumo

Abstract

1 INTRODUÇÃO.

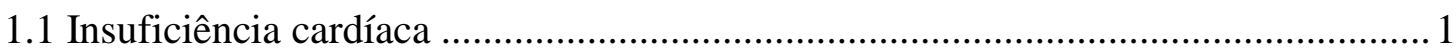

1.2 Alterações hormonais e neurovasculares na insuficiência cardíaca ......................... 3

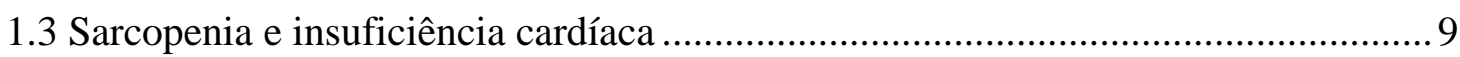

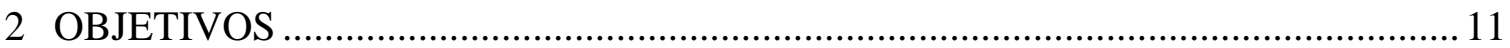

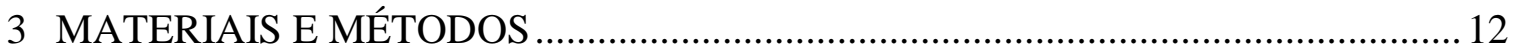

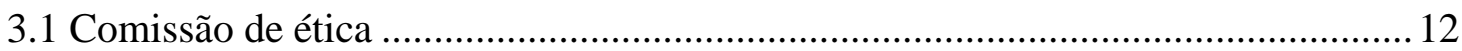

3.2 Amostra, cálculo amostral e critérios de inclusão/exclusão ................................... 12

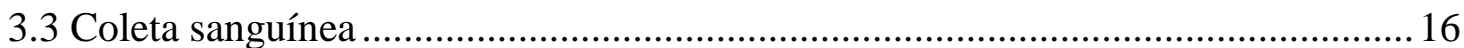

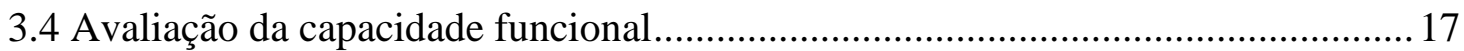

3.5 Avaliação da composição corporal e força muscular ............................................ 18

3.6 Avaliação da atividade nervosa simpática muscular .........................................220

3.7 Avaliação da reatividade parassimpática ..............................................................22

3.8 Avaliação do fluxo sanguíneo muscular do antebraço .......................................... 22

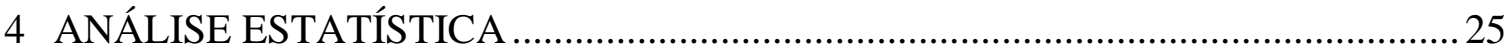

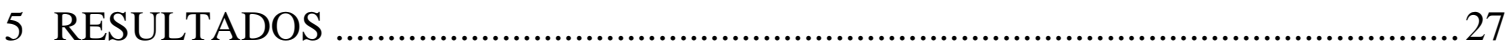

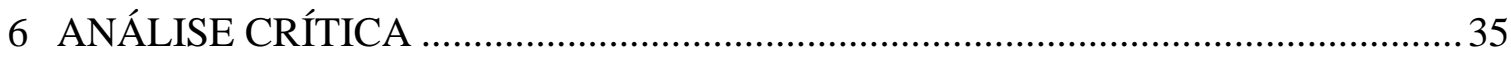


7 CONCLUSÃO

39

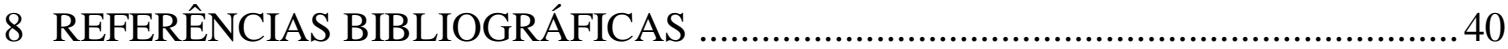

Apêndice: Artigos 1, 2 e 3 


\section{RESUMO}

Fonseca GWP. Efeitos da deficiência anabólica hormonal e alterações neurovasculares no prognóstico de pacientes com insuficiência cardíaca [tese]. São Paulo: Faculdade de Medicina, Universidade de São Paulo; 2020.

INTRODUÇÃO: O impacto da deficiência anabólica hormonal na progressão da insuficiência cardíaca com fração de ejeção reduzida (ICFEr) ainda é controverso, assim como sua interação com alterações neurovasculares. Adicionalmente, embora estas alterações possam levar cronicamente ao quadro de sarcopenia, definido pela perda de força, massa muscular e funcionalidade, a prevalência e os mecanismos específicos da sarcopenia em pacientes com ICFEr são desconhecidos, bem como o efeito da etiologia na composição corporal desses pacientes. OBJETIVOS: Avaliar o efeito da deficiência de hormônios anabólicos sexuais e alterações neurovasculares no prognóstico de pacientes com ICFEr, avaliar a prevalência de sarcopenia e obesidade sarcopênica, assim como o impacto do controle autonômico sobre a sarcopenia nesses pacientes e verificar se há diferença na composição corporal entre pacientes chagásicos com ICFEr e outras etiologias. MÉTODOS: Foram incluídos 169 pacientes com ICFEr (FEVE $<40 \%)$. As concentrações plasmáticas de testosterona livre (TL), estimada pela concentração de testosterona total e globulina ligadora de hormônios sexuais, peptídeo natriurético cerebral (BNP) e demais marcadores sanguíneos foram coletadas no período matutino após um jejum noturno de 12 horas. $\mathrm{O}$ consumo pico de oxigênio $\left(\mathrm{VO}_{2 \text { pico }}\right)$ foi avaliado pelo teste ergoespirométrico realizado em cicloergômetro com incremento progressivo de 5-10W por minuto. A densitometria computadorizada por absorciometria radiológica de dupla energia foi utilizada para mensurar a massa muscular e a massa gorda. A força muscular foi avaliada por um dinamômetro de preensão manual com a mão dominante na posição supinada e o cotovelo flexionado em $90^{\circ}$. A sarcopenia foi definida com base na redução de massa muscular e força de preensão manual. A atividade nervosa simpática muscular (MSNA) foi avaliada através da técnica de microneurografia e a atividade parassimpática pela frequência cardíaca de recuperação do primeiro $(\triangle \mathrm{HRR} 1)$ e segundo minuto $(\triangle \mathrm{HRR} 2)$ immediatamente após esforço máximo. O fluxo sanguíneo muscular do antebraço (FBF) foi medido pela pletismografia de oclusão venosa. RESULTADOS: Em análise multivariada do risco proporcional de Cox, BNP (OR, 3,597; 95\% CI, 1,750-7,392; p<0,001), TL<131 pmol/L (OR, 2,097; 95\% CI, 1,009-4,357; p=0,047) e FBF (OR, 0,568; 95\% CI, 0,273-1,184; $\mathrm{p}=0,031)$ foram preditores independentes de mortalidade, após ajuste para variáveis de confusão. O tempo de sobrevida foi menor em pacientes com TL $<131 \mathrm{pmol} / \mathrm{L}$ e FBF< ou $>1,85 \mathrm{~mL} / \mathrm{min}^{-1} / 100 \mathrm{~mL}^{-1}$ ( $\left.\mathrm{p}<0,001\right)$ em comparação com seus pares com TL normal. A prevalência de sarcopenia foi similar entre pacientes "magros" $(20,8 \%)$ e "obesos" $(21,4 \%)$ quando índices que consideram a massa gorda foram utilizados, sendo que tais índices (Newman e Studenski) foram mais sensíveis para detectar sarcopenia em 
pacientes com índice de massa corporal $\geq 25 \mathrm{~kg} / \mathrm{m}^{2}$. Adicionalmente, pacientes sarcopênicos apresentaram maior MSNA [47 (41-52) vs. 40 (34-48) disparos/min; $\mathrm{p}=0,028]$ com menor $\Delta$ HRR1 [15 (10-21) vs. 22 (16-30) batimentos/min; $\mathrm{p}<0,001] \mathrm{e}$ $\Delta$ HRR2 [25 (19-39) vs. 35 (24-48) batimentos/min, p=0,017] em comparação com pacientes não sarcopênicos, respectivamente. Pacientes com ICFEr de origem chagásica e isquêmica apresentaram menor massa gorda $(16,3 \pm 8,1$ vs. 19,3 $\pm 8,0$ vs. $27,6 \pm 9,4 \mathrm{~kg}$; $\mathrm{p}<0,05)$ e $\mathrm{VO}_{2 \text { pico }}(1,17 \pm 0,36$ vs. $1,15 \pm 0,36$ vs. $1,50 \pm 0,45 \mathrm{~L} / \mathrm{min} ; \mathrm{p}<0,05)$ do que pacientes com cardiomiopatia dilatada, respectivamente, enquanto pacientes chagásicos apresentaram menor força muscular $(27 \pm 8$ vs. $37 \pm 11$ vs. $36 \pm 14 \mathrm{~kg} ; \mathrm{p}<0,05)$ e FBF reduzido $\left(1,84 \pm 0,54\right.$ vs. $2,75 \pm 0,76$ vs. $\left.3,42 \pm 1,21 \mathrm{~mL} / \mathrm{min}^{-1} / 100 \mathrm{~mL}^{-1} ; \mathrm{p}<0,01\right)$ em comparação com pacientes isquêmicos e com miocardiopatia dilatada, respectivamente. CONCLUSÕES: A redução da concentração plasmática de TL associada à diminuição de FBF mostrou ter valor prognóstico em pacientes com ICFEr. Estas alterações estão relacionadas com a progressão da doença e podem levar ao quadro de sarcopenia ou obesidade sarcopênica, que são comorbidades distintas, mas apresentam uma prevalência muito semelhante, em torno de $20 \%$. O desbalanço autonômico exacerbado, evidenciado pelo aumento da MSNA e redução da reatividade vagal após o teste de esforço máximo em pacientes sarcopênicos, pode ser um dos mecanismos envolvidos no desenvolvimento de sarcopenia em pacientes com ICFEr. Ademais, pacientes de etiologia chagásica apresentam piora no FBF, menor força muscular e redução de massa gorda com uma tendência de diminuição da massa muscular, de forma mais precoce, em comparação com as etiologias isquêmica e não isquêmica.

Descritores: Prognóstico; Insuficiência cardíaca; Testosterona; Sarcopenia; Obesidade sarcopênica; Desbalanço autonômico; Pletismografia; Doença de Chagas. 


\begin{abstract}
Fonseca GWP. Effect of androgenic anabolic deficiency and neurovascular alterations on prognosis in patients with heart failure [thesis]. São Paulo: "Faculdade de Medicina, Universidade de São Paulo"; 2020.
\end{abstract}

INTRODUCTION: The impact of androgenic anabolic deficiency on the progression of heart failure with reduced ejection fraction (HFrEF) is controversial, as well as its interaction with neurovascular alterations. Although these changes can chronically lead to sarcopenia, defined by loss of muscle strength, muscle mass and performance, the prevalence and underlying mechanisms of sarcopenia in patients with HFrEF are still unknown, so is the impact of etiology on body composition in these patients. PURPOSE: To assess the impact of androgenic anabolic deficiency and neurovascular alterations on prognosis in patients with HFrEF, to assess the prevalence of sarcopenia and sarcopenic obesity, as well as the impact of autonomic control on sarcopenia and to verify whether there is a difference in body composition between chagasic patients and other etiologies of HFrEF. METHODS: One hundred and sixty nine patients with HFrEF $(\mathrm{LVEF}<40 \%)$ were included. Free testosterone (FT), estimated by total testosterone and sex hormone binding globulin, brain natriuretic peptide (BNP) and other blood variables were collected in the morning after a 12-hour overnight fasting. The peak oxygen consumption $\left(\mathrm{VO}_{2}\right)$ was assessed by the cardiopulmonary exercise test performed on a cycle ergometer with a progressive increment of 5-10W per minute. Dual-energy X-ray absorptiometry was used to measure muscle mass and fat mass. Muscle strength was assessed by a handgrip dynamometer with the dominant hand in the supine position and elbow flexed at $90^{\circ}$. Sarcopenia was defined by reduced muscle mass and handgrip strength. Muscle sympathetic nerve activity (MSNA) was assessed using the microneurography technique and parasympathetic activity was evaluated by heart rate recovery at first $(\triangle \mathrm{HRR} 1)$ and second minute $(\Delta \mathrm{HRR} 2)$ immediately after maximum effort. Forearm blood flow (FBF) was assessed by venous occlusion plethysmography. RESULTS: In a multivariate Cox proportional hazard analysis, BNP (OR, 3.597; 95\% CI, 1.750-7.392; p<0.001), FT<131 pmol/L (OR, 2.097; 95\% CI, 1.009-4.357; $\mathrm{p}=0.047)$ and FBF (OR, 0.568; 95\% CI, 0.273-1.184; $\mathrm{p}=0.031)$ were independently associated with mortality, after adjustment for confounding variables. The survival time was shorter in patients with $\mathrm{FT}<131 \mathrm{pmol} / \mathrm{L}$ and $\mathrm{FBF}<$ or $>1.85$ $\mathrm{mL} / \mathrm{min}^{-1} / 100 \mathrm{~mL}^{-1}(\mathrm{p}<0.001)$ compared to those with FT in the normal range. The prevalence of sarcopenia was similar between "lean" (20.8\%) and "obese" (21.4\%) patients when methods that consider fat mass in its equation were used, while Newman's and Studenski's methods were more accurate to detect sarcopenia in patients with body mass index $\geq 25 \mathrm{~kg} / \mathrm{m}^{2}$. Additionally, sarcopenic patients had a higher MSNA [47 (41-52) vs. 40 (34-48) bursts/min; $\mathrm{p}=0.028$ ] with a lower $\Delta$ HRR1 [15 (10-21) vs. 22 (16-30) beats/min; $\mathrm{p}<0.001$ ] and $\Delta$ HRR2 [25 (19-39) vs. 35 (24-48) beats/min, $\mathrm{p}=0.017$ ] 
compared to non-sarcopenic patients, respectively. Patients with HFrEF from Chagas disease and ischemic origin had lower fat mass (16.3 \pm 8.1 vs. $19.3 \pm 8.0$ vs. $27.6 \pm 9.4 \mathrm{~kg}$; $\mathrm{p}<0.05)$ and peak $\mathrm{VO}_{2}(1.17 \pm 0.36$ vs. $1.15 \pm 0.36$ vs. $1.50 \pm 0.45 \mathrm{~L} / \mathrm{min} ; \mathrm{p}<0.05)$ than patients with dilated cardiomyopathy, respectively, whereas chagasic patients presented impaired muscle strength $(27 \pm 8$ vs. $37 \pm 11$ vs. $36 \pm 14 \mathrm{~kg} ; \mathrm{p}<0.05)$ and reduced FBF (1.84 \pm 0.54 vs. $2.75 \pm 0.76$ vs. $\left.3.42 \pm 1.21 \mathrm{~mL} / \mathrm{min}^{-1} / 100 \mathrm{~mL}^{-1} ; \mathrm{p}<0.01\right)$ compared to patients with ischemia and dilated cardiomyopathy, respectively. CONCLUSION: Reduced FT associated with decreased FBF has a prognostic value in patients with HFrEF. These changes may be related to the progression of HFrEF and lead chronically to sarcopenia and sarcopenic obesity, which are distinct comorbidities, but they have a similar prevalence, approximately 20\%. Exacerbated sympatho-vagal imbalance, characterized by increased MSNA and blunted vagal reactivation in sarcopenic patients, may be one of the mechanisms involved in the development of sarcopenia in patients with HFrEF. In addition, patients with Chagas disease have decreased FBF, impaired muscle strength and reduced fat mass with a tendency to have a reduction in muscle mass earlier compared to ischemic and non-ischemic etiologies.

Descriptors: Prognosis; Heart failure; Testosterone; Sarcopenia; Sarcopenic obesity; Autonomic imbalance; Plethysmography; Chagas disease. 


\section{INTRODUÇÃO}

\subsection{Insuficiência cardíaca}

A insuficiência cardíaca (IC) é definida como uma síndrome crônica e sistêmica que promove alterações morfológicas e funcionais no miocárdio, resultando em uma redução do débito cardíaco e uma oferta insuficiente de sangue e oxigênio para os tecidos periféricos em repouso e durante estresse ${ }^{1,2}$. Os sintomas clássicos da IC são dispneia e fadiga exacerbada que impactam significativamente a qualidade de vida dos pacientes e podem ser acompanhados de sinais como hipotensão severa, má perfusão periférica, congestão pulmonar, ortopneia, elevação da pressão venosa jugular e edema periférico ${ }^{3}$.

Embora, os sinais e sintomas da IC sejam semelhantes entre as etiologias, as bases fisiopatológicas diferem ${ }^{1}$. Entre as causas mais comuns de IC podem-se listar a doença arterial coronariana, doença hipertensiva, cardiomiopatia dilatada idiopática, doenças valvares, doença de Chagas, congênitas, arritmias ventriculares complexas, cardiotoxicidade por drogas, fármacos ou álcool e miocardite ${ }^{1}$.

A IC pode ser classificada de acordo com o sistema circulatório afetado (ventrículo direito ou esquerdo), função cardíaca (sistólica ou diastólica) e fatores fisiopatológicos de sobrecarga ventricular (pressão ou volume), com possível sobreposição dessas definições ${ }^{1}$. Essas alterações levam à IC com fração de ejeção do ventrículo esquerdo reduzida (ICFEr; FEVE<40\%), intermediária (ICFEi; FEVE 4049\%) ou preservada (ICFEp; FEVE $\geq 50 \%$ ), de acordo com a última diretriz Brasileira de IC crônica e aguda ${ }^{3}$. Adicionalmente, a classificação funcional proposta pela New York Heart Association (NYHA) é um parâmetro clínico amplamente utilizado para avaliar o grau de tolerância ao esforço e disfunção ventricular de pacientes com IC. 
Na população geral, a prevalência de ICFEr é em torno de 1-2\% em países desenvolvidos, podendo chegar até $10 \%$ em pessoas com idade superior a $65 \operatorname{anos}^{4-6}$. A maior prevalência de ICFEr ocorre no sexo masculino que apresenta um desfecho piorado em comparação às mulheres ${ }^{7}$, normalmente diagnosticadas em idades mais avançadas ${ }^{5}$. Ademais, a chance de um indivíduo desenvolver IC ao longo da vida a partir de 55 anos é de $33 \%$ para homens e $28,5 \%$ para mulheres ${ }^{5}$.

Apesar da incidência de IC ser proporcional ao aumento da idade para ambos os $\operatorname{sexos}^{4,5}$, os homens apresentam uma maior incidência do que as mulheres ${ }^{5}$. Em países desenvolvidos, a incidência de ICFEr tem diminuído em decorrência do maior controle de fatores de risco, e em contrapartida tem ocorrido um aumento na incidência de ICFEp devido a melhora na expectativa de vida ${ }^{8}$. Este cenário epidemiológico, no entanto, não é reproduzido em países subdesenvolvidos, uma vez que os pacientes são mais jovens e acometidos por outras etiologias como a doença de Chagas 9 .

As bases fisiopatológicas da ICFEr estão definidas na literatura, contudo, o entendimento da interação entre os mecanismos fisiopatológicos que levam ao dano cardíaco, direto ou indireto, carece de maior esclarecimento. A remodelação cardíaca a partir da agressão direta aos cardiomiócitos e à matriz extracelular compromete o esqueleto fibroso do miocárdio, bem como a dinâmica de acoplamento dos sarcômeros ${ }^{1}$. Além disso, a disfunção cardíaca pode ocorrer por meio de um evento cardíaco agudo (p. ex. infarto agudo do miocárdio) ou mudanças dismórficas crônicas que alteram a relação contração/enchimento, levando ao aumento do tamanho do coração, assim como, redução da função do ventrículo esquerdo como bomba. A ativação de mecanismos neuro-humorais, vasculares, inflamatórias, imunológicas, aumento do estresse oxidativo, redução do suprimento energético do miocárdio e apoptose são inerentes ao complexo de desencadeamento e agravamento da $\operatorname{ICFEr}^{10}$. Estas alterações 
são responsáveis por intensificarem o remodelamento cardíaco que leva a um impacto sistêmico, uma vez que reduz o fluxo sanguíneo periférico e aumenta a vasoconstrição para os órgãos e tecidos (pulmões, rins, músculos esqueléticos, fígado, etc.), gerando assim uma intolerância ao mínimo esforço físico realizado e perpetuando o ciclo fisiopatológico vicioso que contribui para a progressão da síndrome ${ }^{6}$.

Apesar dos avanços obtidos no tratamento da ICFEr, a mortalidade por essa causa ainda é alta e pode variar entre $24 \%$ para pacientes com idade até 60 anos e $54 \%$ naqueles acima de $80 \operatorname{anos}^{8}$. Além das comorbidades comumente associadas ao pior prognóstico de pacientes com ICFEr, tais como diabetes mellitus ${ }^{11}$, dislipidemia ${ }^{12}$, obesidade $^{13}$, anemia ${ }^{14}$, disfunção renal ${ }^{15}$, apneia obstrutiva do sono ${ }^{16}$ e doença pulmonar obstrutiva crônica $^{17}$, a deficiência de hormônios anabólicos ${ }^{18}$, a hiperatividade simpática $^{19}$ e a sarcopenia ${ }^{20}$ têm sido associadas com uma maior mortalidade nessa população.

\subsection{Alterações hormonais e neurovasculares na insuficiência cardíaca}

Alterações da função cardíaca levam a inúmeras compensações neuro-humorais características da ICFEr. Essas adaptações agudas são de extrema importância para manter a integridade da função do miocárdio, mas, por outro lado, trazem consequências deletérias quando se tornam crônicas. Diversos estudos têm evidenciado que a ICFEr, além das alterações neuro-humorais, também desencadeia alterações endócrinas e neurovasculares que podem piorar a progressão da doença e o prognóstico dos pacientes ${ }^{18,19,21}$.

Essas evidências apontam para mudanças na secreção e ação de diferentes hormônios, como o hormônio do crescimento $(\mathrm{GH})$, hormônio estimulante da tireoide (TSH), cortisol, tri-iodotironina (T3), tiroxina (T4), insulina e, em especial, os 
hormônios androgênicos derivados da testosterona ${ }^{22-29}$. Essas alterações afetam a homeostase proteica do organismo com maior exacerbação de processos catabólicos e redução de processos anabólicos, produzindo assim um desbalanço entre anabolismo e catabolismo em pacientes com ICFEr que pode levar ao desenvolvimento de $\operatorname{miopatias}^{30}$.

A testosterona, hormônio anabólico derivado do colesterol, é o hormônio androgênico mais importante secretado pelas gônadas masculinas, o qual exerce duas funções principais no organismo: (1) desenvolvimento e manutenção das características sexuais masculinas (função androgênica), e (2) crescimento e desenvolvimento dos músculos esqueléticos e ossos (função anabólica).

O hipotálamo secreta pulsatilmente a cada 60-90 minutos, o hormônio liberador de gonadotrofina $(\mathrm{GnRH})$ que estimula a hipófise anterior a produzir e liberar o hormônio folículo estimulante (FSH) e o hormônio luteinizante (LH). Nas gônadas, o FSH estimula as células de Sertoli na produção de espermatozoide, enquanto o LH estimula as células de Leydig responsáveis pela produção de testosterona ${ }^{31}$. A testosterona então age diretamente nos receptores androgênicos localizados no músculo esquelético e cardíaco $^{32}$ por meio da conversão de dois metabólicos ativos, a dihidrotestosterona e o estradiol, pela ação das enzimas 5- $\alpha$-redutase e aromatase, respectivamente.

As células de Leydig, situadas no interstício do testículo maduro, produzem aproximadamente $95 \%$ da testosterona total (TT; eixo hipotálamo-hipófise-gonadal), e outros 5\% correspondem à liberação de dehidroepiandrosterona sulfato (DHEA-S), produzida na zona reticular das glândulas adrenais (eixo hipotálamo-hipófise-adrenal), as quais são estimuladas pelo hormônio adrenocorticotrófico (ACTH) secretado pela hipófise anterior. De uma forma complementar, há um terceiro eixo responsável pela 
resposta anabólica (eixo somatotrófico), onde ocorre a secreção do hormônio liberador de hormônio do crescimento (GHRH) pelo hipotálamo. Este, por sua vez, estimula a hipófise anterior a liberar GH, que no fígado produzirá o fator de crescimento semelhante à insulina tipo 1 (IGF-1). O IGF-1 também exerce efeitos similares à insulina, promovendo síntese proteica no músculo esquelético.

Os testículos produzem em torno de 3 a $10 \mathrm{mg}$ de testosterona diariamente que correspondem a uma concentração plasmática de 10,4-34,7 nmol/L, obtendo seu pico no período matutino ${ }^{31}$. Por ser um hormônio esteroide derivado de lipoproteínas de baixa densidade (LDL), o transporte sanguíneo depende de proteínas carreadoras, onde 58\% da TT estão ligadas à albumina, 40\% ligadas à globulina ligadora de hormônios sexuais (SHBG) e apenas $2 \%$ estão na sua forma livre, sendo esta quantidade responsável, de fato, pela resposta biológica direta nos tecidos alvos.

Em estudos comparativos entre pacientes com IC e indivíduos saudáveis pareados pela idade, as concentrações plasmáticas de TT, testosterona livre (TL) e DHEA-S mostravam-se reduzidas, enquanto as concentrações plasmáticas de SHBG, LH e FSH estavam aumentadas ${ }^{33}, 34$. Os resultados desses estudos sugerem que em pacientes com IC ocorre uma supressão na secreção de hormônios anabólicos pelo eixo gonadal e adrenal, o que leva ao aumento reflexo nos hormônios gonadotróficos, caracterizando um quatro de hipogonadismo primário.

Embora não exista um ponto de corte estabelecido para definição de hipogonadismo, valores inferiores a 9,7-10,4 nmol/L para TT e $0,17-0,31 \mathrm{nmol} / \mathrm{L}$ para TL são utilizados como definição de limites de normalidade ${ }^{31,35}$. Ainda assim, admitese que a prevalência de hipogonadismo em pacientes com IC seja em torno de $25-30 \%{ }^{28}$, 36 
A deficiência anabólica hormonal, definida pela redução na secreção de hormônios em apenas um ou mais eixos anabólicos, pode estar relacionada com a redução da massa e da força muscular, denominada clinicamente como sarcopenia e caquexia cardíaca $^{29}$. Outro possível mecanismo responsável por essa redução de massa muscular é um aumento de marcadores inflamatórios, tais como: fator de necrose tumoral (TNF) e interleucina-6 (IL-6) ${ }^{29,37}$. Em estudo experimental com ratos, ficou demonstrado que um aumento significativo de TNF foi capaz de inibir a secreção de testosterona pelas células de Leydig $^{38}$. Adicionalmente, alterações nos eixos anabólicos, associadas ou não, foram relacionadas com uma redução em marcadores de capacidade funcional como o consumo pico de oxigênio $\left(\mathrm{VO}_{2 \text { pico }}\right)$ e a razão ventilação/produção de dióxido de carbono $\left(\mathrm{VE} / \mathrm{VCO}_{2}\right)^{36,39}$.

A deficiência anabólica hormonal em pacientes com ICFEr também apresenta associação com um aumento significativo de peptídeo natriurético cerebral (BNP), importante marcador de pior prognóstico nesta população ${ }^{34,36}$. Ademais, pacientes com ICFEr e deficiência anabólica hormonal, quando comparados com seus pares sem deficiência, apresentam maior tempo de internação e uma maior frequência de readmissão ${ }^{40,41}$. Diversos estudos também têm demonstrado o impacto da deficiência anabólica hormonal sobre a mortalidade de pacientes com ICFEr, independente do eixo anabólico acometido ${ }^{40,41}$. Jankowska e colaboradores demonstraram que múltiplas deficiências anabólicas hormonais no eixo gonadal (TT e TL), adrenal (DHEA-S) e somatotrófico (IGF-1) estavam relacionados com um pior prognóstico nesta população. Além disso, a adição de eixos anabólicos afetados mostrou um impacto na sobrevida de pacientes com ICFEr em 74\%, 55\% e 27\% para 1, 2 e 3 eixos, respectivamente, em um período de três anos de acompanhamento ${ }^{18}$. Entretanto, a redução nas concentrações plasmáticas de testosterona, como marcador independente de mortalidade, ainda é 
controverso, enquanto alguns estudos confirmam esse achado ${ }^{18,42,43}$, outros não conseguiram demonstrar tal associação ${ }^{44,45}$.

Outra função frequentemente atribuída à testosterona é seu efeito vasodilatador, através de uma redução do perfil inflamatório, modulação dos canais de cálcio e liberação de óxido nítrico, favorecendo a melhora da função endotelial ${ }^{46,}{ }^{47}$. Além do mais, a testosterona pode exercer efeito sobre o controle autonômico através dos barorreceptores $^{48}$. Em pacientes com ICFEr, a disfunção barorreflexa crônica leva a uma maior liberação de vasopressina, aldosterona e angiotensina II, alterando as respostas reflexas de mecanorreceptores, metaborreceptores e quimiorreceptores ${ }^{49-51}$. Desse modo, o balanço entre fatores de inibição e excitação simpática tem como resultado uma condição permanente de pró-excitação simpática em pacientes com ICFEr.

O sistema nervoso simpático (SNS), por meio das fibras pós-ganglionares, libera noradrenalina que atua nos receptores do tipo alfa e beta-adrenérgicos, localizados predominantemente nos vasos sanguíneos, coração e pulmões ${ }^{52}$. Com o avançar da idade é observado um aumento natural na atividade do SNS em indivíduos saudáveis, e este aumento parece ser mais acentuado em homens do que em mulheres ${ }^{53}$. A atuação do SNS é essencial no controle e regulação dos reflexos cardiovasculares em condições de repouso e estresse ${ }^{50}$. A atividade nervosa simpática muscular (MSNA) pode ser avaliada pela técnica de microneurografia, um método que avalia o arco reflexo eferente do SNS para o sistema musculoesquelético, por meio do posicionamento de um microeletrodo no nervo fibular ${ }^{54}$. Tal método tem se mostrado mais específico para avaliar a descarga adrenérgica característica em pacientes com ICFEr e é correlacionado com elevações nas concentrações plasmáticas de noradrenalina ${ }^{55,56}$. 
O aumento da MSNA em pacientes com ICFEr é independente de idade, sexo e etiologia $^{51,56,57}$. Este aumento reflete a progressão na falência da bomba cardíaca e apresenta-se como fator de pior prognóstico desses pacientes ${ }^{19}$. No entanto, uma análise adicional que parece complementar o valor prognóstico da MSNA nesses pacientes é a avaliação de fluxo sanguíneo muscular, realizada por meio da técnica de pletismografia de oclusão venosa, que permite mensurar o fluxo sanguíneo muscular do antebraço $(\mathrm{FBF})^{58}$. Pacientes com ICFEr, quando comparados com indivíduos saudáveis, apresentam uma redução significativa do $\mathrm{FBF}^{59}$.

A redução do FBF em pacientes com ICFEr depende principalmente do balanço entre os mecanismos periféricos de vasodilatação (liberação de óxido nítrico) e vasoconstrição (aumento da MSNA). Além do mais, após bloqueio de mecanismos endotélio dependente e independente, reduções da vasodilatação durante exercício estático de preensão manual e estresse mental foram relacionadas com um aumento da MSNA em pacientes com ICFEr, sugerindo que a MSNA controle ambos os mecanismos ${ }^{59-61}$. Adicionalmente, as consequências funcionais deste aumento exacerbado da MSNA são evidenciadas por uma relação inversamente proporcional com a redução do $\mathrm{VO}_{2 \text { pico }}{ }^{62,63}$.

Em estudo experimental com ratos, Bacurau e colaboradores também demonstraram que o aumento da MSNA promoveu a redução de massa muscular ${ }^{64}$. Adicionalmente, pacientes com IC e sarcopenia apresentam disfunção endotelial piorada em comparação com pacientes sem sarcopenia ${ }^{65}$. Desta forma, o aumento exacerbado da MSNA associado a redução do FBF reduz o aporte sanguíneo de nutrientes para a musculatura ativa, gerando assim, uma redução da massa muscular e significativa intolerância ao esforço em pacientes com ICFEr. 
Entretanto, o impacto da interação entre alterações hormonais e neurovasculares na mortalidade de pacientes com ICFEr não está bem elucidada na literatura, assim como a influência dessas alterações na composição corporal dessa população.

\subsection{Sarcopenia e insuficiência cardíaca}

A sarcopenia é definida pela redução de massa muscular associada ao declínio na força muscular ou desempenho físico decorrente do processo de envelhecimento ${ }^{66}$. O termo sarcopenia é derivado do grego, onde Sarx significa "carne" e penia significa "perda"67. Em essência, a sarcopenia pode ser entendida com uma falência de órgão, uma vez que representa uma insuficiência muscular em manter a contratação muscular por falta de componente contrátil (massa muscular) ou por inadequada eficiência contrátil (força/resistência muscular).

Em pacientes com IC, a prevalência de sarcopenia pode variar de $19,5 \%^{37}$ a $47,3 \%{ }^{68}$. Embora o avanço da idade seja o principal fator para o desenvolvimento de sarcopenia, a progressão da IC pode levar ao aparecimento precoce dessa comorbidade em pacientes mais jovens ${ }^{68}$.

A fisiopatologia da sarcopenia é multifatorial e parece ocorrer por um desbalanço entre a síntese proteica e a degradação muscular ${ }^{30}$. Entre os principais mecanismos envolvidos no desenvolvimento e progressão da sarcopenia podemos citar a disfunção mitocondrial ${ }^{69}$, lipotoxidade muscular, aumento de marcadores inflamatórios $^{70}$, alterações neurológicas ${ }^{71}$, redução na concentração de hormônios anabólicos $^{72}$, inatividade física ${ }^{73}$, redução do fluxo sanguíneo muscular $^{65}$ e complicações digestivas ${ }^{74}$.

Embora, pacientes com ICFEr compartilhem alguns dos mecanismos descritos em pacientes sarcopênicos, o impacto de alterações características da ICFEr como o 
desbalanço autonômico, determinado pelo aumento da atividade nervosa simpática e redução da atividade nervosa parassimpática ${ }^{75}$, sobre o desenvolvimento de alterações na composição corporal não é conhecido. Adicionalmente, a etiologia da IC, seja ela de origem isquêmica ou não, pode estimular o processo de perda de massa muscular.

Entretanto, o clássico fenótipo do paciente sarcopênico com o corpo franzino tem sido alterado devido ao aumento na incidência de sobrepeso e obesidade na população. Esse fenômeno caracterizado pela combinação na redução de massa muscular e aumento de gordura corporal tem sido denominado obesidade sarcopênica e deve-se, principalmente, à convergência entre o envelhecimento populacional e o aumento das taxas de obesidade ${ }^{76}$. Ademais, a sobreposição dessas comorbidades pode gerar um grupo de pacientes com mais complicações ${ }^{77,78}$, visto que ambas apresentam um impacto negativo no prognóstico de pacientes com IC. Dessa maneira, o índice mais utilizado na prática clínica para definir a sarcopenia, derivado da massa muscular apendicular de membros superiores e inferiores dividida pela altura ao quadrado, não inclui a massa gorda em sua formulação, o que pode subestimar a prevalência de sarcopenia em pacientes com sobrepeso e obesidade.

Portanto, a prevalência de obesidade sarcopênica em pacientes com ICFEr é desconhecida, de igual modo o impacto da etiologia e disfunção autonômica no desenvolvimento de sarcopenia nesses pacientes. 


\section{OBJETIVOS}

\section{Objetivo primário:}

1) Avaliar o efeito da deficiência de hormônios anabólicos sexuais e alterações neurovasculares no prognóstico de pacientes com ICFEr.

Objetivos secundários:

1) Verificar a prevalência de sarcopenia e obesidade sarcopênica em pacientes com ICFEr a partir da distribuição da composição corporal;

2) Avaliar o impacto do controle autonômico sobre a sarcopenia em pacientes com ICFEr;

3) Verificar se há diferença na composição corporal e fluxo sanguíneo muscular periférico entre pacientes com ICFEr de diferentes etiologias. 


\section{MATERIAIS E MÉTODOS}

\subsection{Comissão de ética}

O presente projeto foi aprovado pelo comitê de ética e pesquisa do Hospital das Clínicas da Faculdade de Medicina da Universidade de São Paulo (HCFMUSP; Comissão de Ética para Análise de Projetos de Pesquisa (CAPPesq) - No 0892/07). Os aspectos éticos foram preservados de acordo com a Resolução do Conselho Nacional de Saúde nº 196, de 10 de outubro de 1996 e a Declaração de Helsinque de 2013.

\subsection{Amostra, cálculo amostral e critérios de inclusão/exclusão}

O desenho do estudo foi de coorte prospectivo e observacional com a inclusão de 169 pacientes do sexo masculino com ICFEr, acompanhados por um período mínimo de 36 meses (Figura 1). Os pacientes foram recrutados na Unidade Clínica de Miocardiopatias do Instituto do Coração (InCor) do HCFMUSP e no Ambulatório de Cardiologia Esportiva e Reabilitação Cardíaca do InCor do HCFMUSP. Os pacientes assinaram o termo de consentimento livre e esclarecido, após esclarecimento verbal sobre os procedimentos e riscos envolvidos no estudo, em duas vias, uma das quais foi entregue ao paciente e a outra ficou arquivada. O estudo foi registrado no website ClinicalTrials.gov utilizando o acrônimo TESTO-HF (TESTOsterone - Heart Failure) com o identificador NCT03463226.

O cálculo amostral foi baseado no estudo de Jankowska e colaboradores ${ }^{18}$, que correlacionaram a classe funcional e a deficiência hormonal anabólica com a mortalidade em 208 pacientes com IC, e no estudo do grupo em que Santos e colaboradores observaram o risco aumentado de morte e taxa de hospitalização com a presença de deficiência hormonal anabólica em pacientes com $\mathrm{IC}^{41}$. Com base nesses 
dois estudos, foi utilizado o cálculo amostral de variação da média do intervalo de confiança dos níveis plasmáticos de TT. Dessa maneira, a projeção de aproximadamente 47 pacientes por classe funcional foi determinada da seguinte forma:

Sample size for a descriptive study of a continuous variable (TT)

\begin{tabular}{|c|c|c|c|}
\hline$C L=$ & 95 & $\%$ & Confidence level (95\%) \\
\hline$W=$ & 2.77 & $\langle=" \prime \prime\rangle$ & Desired total width of confidence interval \\
\hline & 4.85 & & Standard deviation of the variable \\
\hline
\end{tabular}

$W / S=0.57$

Standard normal deviate for $\alpha=Z_{\alpha}=1.96$

Sample size $=N=4 Z_{a}{ }^{2} S^{2} / W^{2}=47$

De acordo com a classe funcional, os pacientes foram alocados da seguinte maneira:

Grupo 1 (n=54): classe funcional I $(N Y H A I)$;

Grupo 2 (n=57): classe funcional II (NYHA II);

Grupo 3 (n=46): classe funcional III (NYHA III);

Grupo 4 (n=12): classe funcional IV (NYHA IV).

Para a participação no estudo, os pacientes deveriam apresentar os seguintes critérios:

\section{A) Critérios de inclusão:}

$\checkmark$ Homens com idade entre 18 e 65 anos; 
Classe Funcional I, II, III e IV;

Etiologia: isquêmica, hipertensiva, chagásica e idiopática;

$\checkmark$ Diagnóstico de ICFEr há pelo menos um ano (FEVE<40\%);

$\checkmark$ Clinicamente estável e com otimização da medicação por pelo menos um mês.

\section{B) Critérios de exclusão:}

Infarto agudo do miocárdio com angioplastia ou revascularização há pelo menos seis meses do estudo;

$\checkmark$ Qualquer tratamento hormonal no momento do estudo ou no passado;

$\checkmark$ Presença de distrofia muscular por degeneração neural (p. ex. distrofia muscular de Duchenne);

$\checkmark$ Presença de marca-passo ou ressincronizador;

$\checkmark$ Histórico de câncer;

$\checkmark$ Presença de insuficiência cardíaca congestiva descompensada;

$\checkmark$ Presença de neuropatia autonômica periférica e diabetes mellitus tipo I ou tipo II insulino-dependente;

$\checkmark$ Doença renal crônica dialítica. 
Revisão de prontuários: Pacientes com ICFEr de acordo com os critérios de inclusão/exclusão

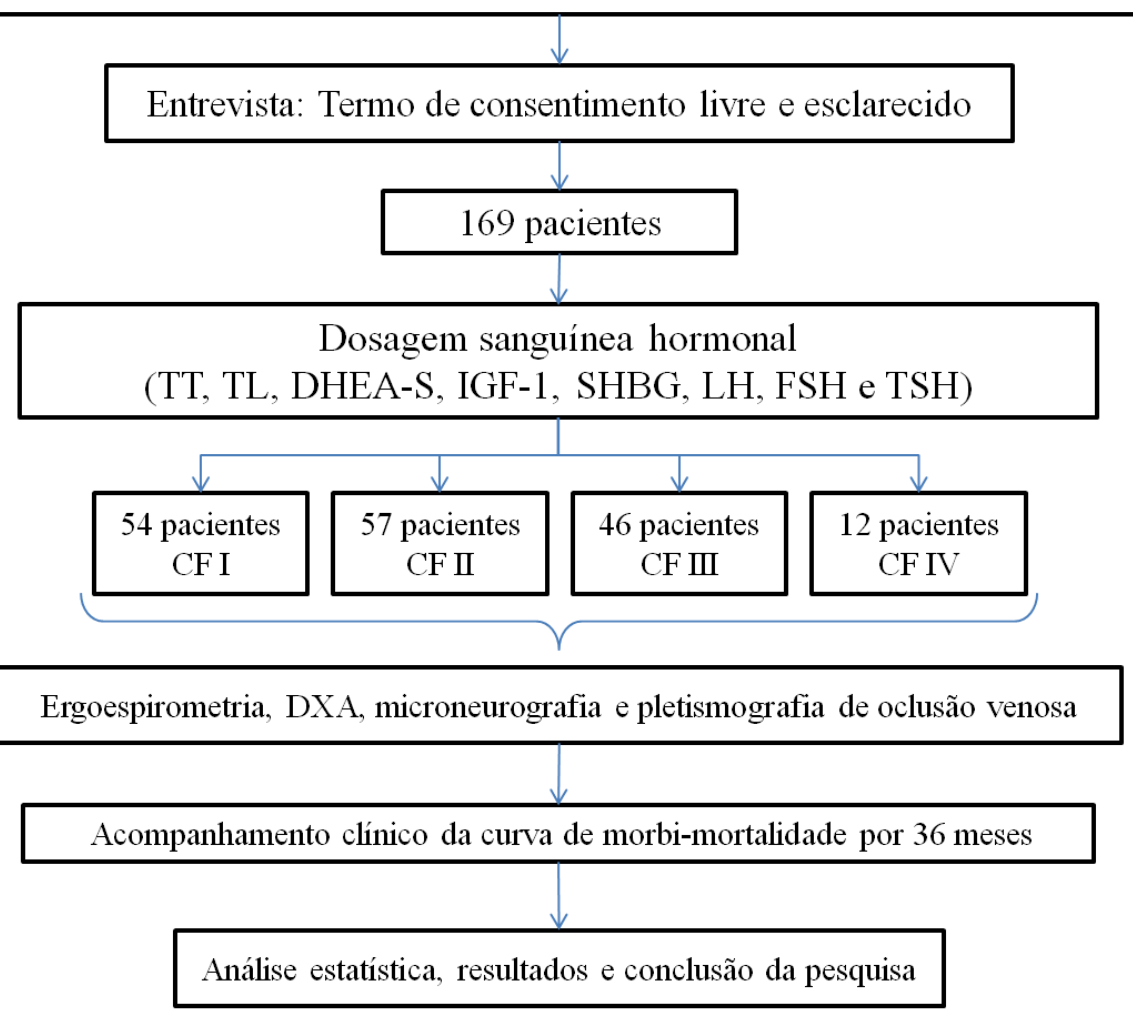

Figura 1. Fluxograma do estudo. $\mathbf{C F}=$ classe funcional; DHEA-S $=$ dehidroepiandrosterona sulfato; DXA = densitometria computadorizada por absorciometria radiológica de dupla energia; FSH = hormônio folículo estimulante; $\mathbf{I C F E r}=$ insuficiência cardíaca com fração de ejeção reduzida; IGF-1 = fator de crescimento semelhante à insulina tipo 1; LH = hormônio luteinizante; $\mathbf{S H B G}=$ globulina ligadora de hormônios sexuais; $\mathbf{T L}=$ testosterona livre; $\mathbf{T S H}=$ hormônio estimulante da tireoide; $\mathbf{T T}=$ testosterona total. 


\subsection{Coleta sanguínea}

As amostras de sangue foram coletadas entre às 7 e 11 horas da manhã, após um jejum noturno de 12 horas. As análises das variáveis hormonais foram realizadas no laboratório de Hormônios e Genética Molecular da Disciplina de Endocrinologia do HCFMUSP. As concentrações séricas de TT (coeficiente de variação: intra-ensaio de 4,7\% e inter-ensaio de 8,4\%), SHBG (coeficiente de variação: intra-ensaio de 2,1\% e inter-ensaio de 2,7\%), DHEA-S (coeficiente de variação: intra-ensaio de 2,8\% e interensaio de 3,6\%), IGF-1 (coeficiente de variação: intra-ensaio de 2,0\% e inter-ensaio de 3,6\%), FSH (coeficiente de variação: intra-ensaio de 1,8\% e inter-ensaio de 5,3\%), LH (coeficiente de variação: intra-ensaio de 1,8\% e inter-ensaio de 5,2\%) e TSH (coeficiente de variação: intra-ensaio de $8,6 \%$ e inter-ensaio de 8,7\%) foram determinados utilizando os analisadores de imunoensaios Elecsys e Cobas e. As concentrações plasmáticas de TT e SHBG foram utilizadas para estimar a concentração plasmática de TL, usando a fórmula validada e proposta por Vermeulen e colaboradores $^{79}$. O hipogonadismo foi definido quando ambas as concentrações plasmáticas de TT e TL estavam abaixo de $300 \mathrm{ng} / \mathrm{dL}$ e 131 pmol/L, respectivamente ${ }^{80}$, ${ }^{81}$. O valor de hemoglobina $<13 \mathrm{~g} / \mathrm{dL}$ foi utilizado para a definição de anemia.

Artigos 1, 2 e 3. Os exames laboratoriais clínicos incluíram sódio e potássio sérico $(\mathrm{mEq} / \mathrm{L})$, BNP $(\mathrm{pg} / \mathrm{mL})$, creatinina $(\mathrm{mg} / \mathrm{dL})$, uréia $(\mathrm{mg} / \mathrm{dL})$, hemoglobina plasmática $(\mathrm{g} / \mathrm{dL}$ ), proteína C-reativa ultra sensível (hs-CRP; mg/L), triglicérides (mg/dL), colesterol total $(\mathrm{mg} / \mathrm{dL})$, lipoproteína de alta densidade (HDL; mg/dL), lipoproteína de baixa densidade (LDL; mg/dL) e glicemia em jejum (mg/dL). 


\subsection{Avaliação da capacidade funcional}

Artigos 1, 2 e 3. Após tricotomia e assepsia do local onde os eletrodos foram posicionados, o eletrocardiograma de doze derivações (Cardio Soft 6.51 ECG/CAM-14, GE Medical Systems Information Technologies, Wisconsin, WI, USA) foi realizado para avaliar o padrão eletrocardiógrafo dos pacientes em repouso, durante o esforço e na fase de recuperação do teste ergoespirométrico. A frequência cardíaca (FC) foi registrada continuamente do repouso até a fase de recuperação do teste. A pressão arterial (PA) foi medida pelo método auscultatório em repouso com o paciente na posição sentada, a cada dois minutos durante a fase de esforço e a cada minuto na fase de recuperação do teste.

Os pacientes realizaram o teste ergoespirométrico (Figura 2) em cicloergômetro eletromagnético (Ergometer 800S; Sensor Medics, Yorba Linda, CA, USA), seguindo protocolo de rampa com aumentos incrementais de carga de 5-10 W por minuto até a exaustão voluntária máxima. Os pacientes foram instruídos a pedalar em uma velocidade média de 60-70 rotações por minuto (rpm). A escolha do protocolo foi definida a partir do nível de tolerância do paciente aos esforços diários e valor predito de $\mathrm{VO}_{2}$ máximo de acordo com a idade ${ }^{82}$. $\mathrm{O} \mathrm{VO}_{2 \text { pico }}$ foi considerado como o consumo de oxigênio obtido no pico do esforço, quando o paciente, apesar de encorajamento verbal, não conseguisse manter a velocidade mínima da bicicleta de 60 rpm.

A ventilação pulmonar (VE), concentração de oxigênio $\left(\mathrm{O}_{2}\right)$, concentração de dióxido de carbono $\left(\mathrm{CO}_{2}\right)$, quociente respiratório $(\mathrm{QR})$, equivalentes ventilatórios de $\mathrm{VO}_{2}$ e $\mathrm{VCO}_{2}\left(\mathrm{VE} / \mathrm{VO}_{2}\right.$ e $\mathrm{VE} / \mathrm{VCO}_{2}$, respectivamente) foram analisadas a cada ciclo respiratório por um espirômetro computadorizado (Vmax Encore 29 System; VIASYS 
Healthcare Inc., Palm Springs, CA, USA ${ }^{83}$. O QR $\geq 1,10$ foi atingido por $82 \%$ dos pacientes.

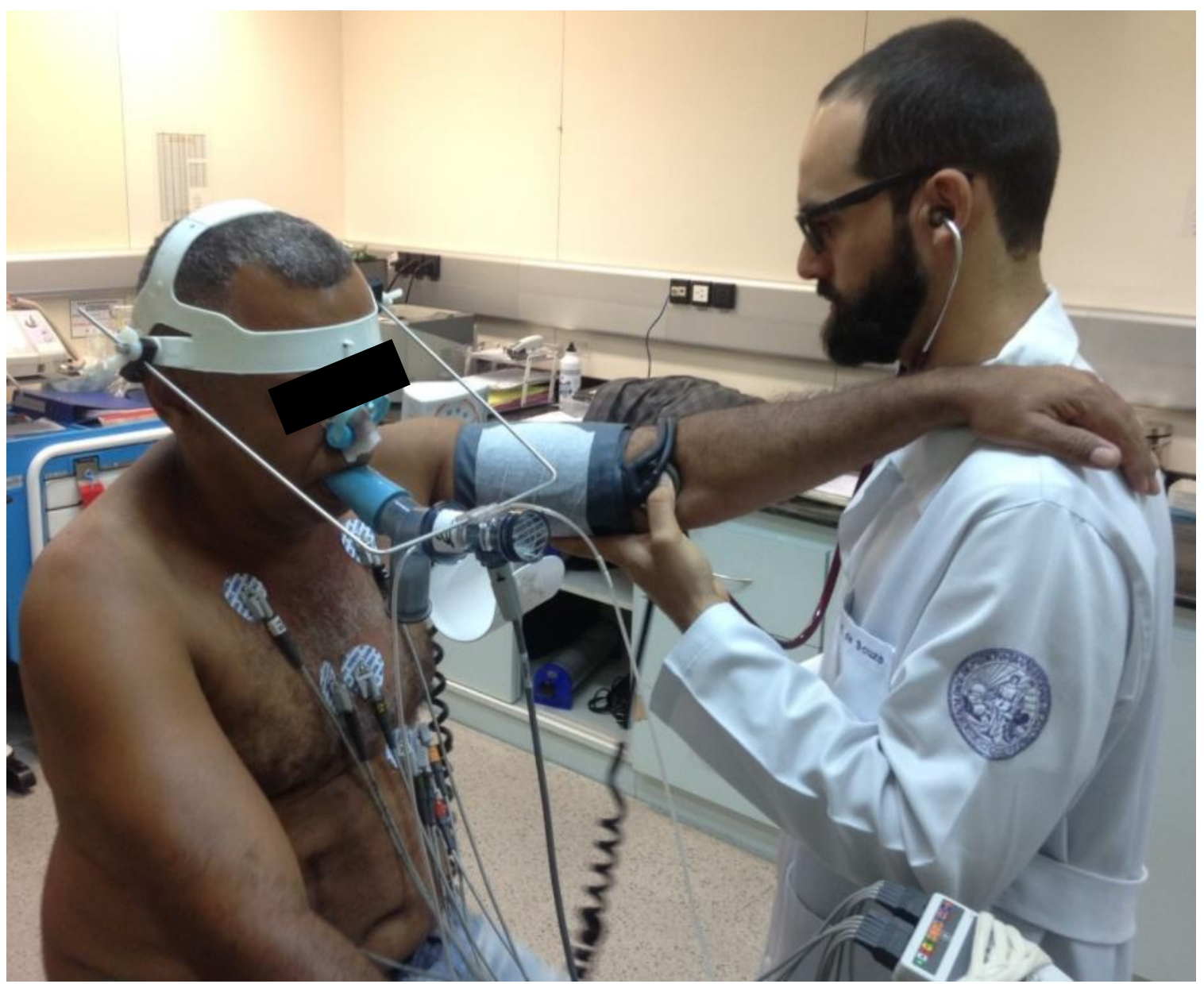

Figura 2. Avaliação da capacidade funcional em cicloergômetro - Teste ergoespirométrico.

\subsection{Avaliação da composição corporal e força muscular}

Artigos 1, 2 e 3. O método de densitometria computadorizada por absorciometria radiológica de dupla energia (DXA; Figura 3) (Lunar iDXA; GE Medical Systems Lunar, Madison, WI, USA) foi utilizado para quantificar a composição corporal através de um procedimento de imagem, determinando conteúdo e densidade mineral óssea, massa muscular e massa gorda. A DXA emite ondas eletromagnéticas provenientes de dois picos distintos de energia de isótopos de alta afinidade e baixa intensidade - gadolínio $153\left({ }^{153} \mathrm{Gd}\right)$, que penetram nas áreas ósseas e de tecidos moles 
com uma profundidade de aproximadamente 30 centímetros. $\mathrm{O}$ exame foi realizado com o paciente em decúbito dorsal sobre uma mesa, onde a fonte passou sob os segmentos, escaneando o corpo inteiro com uma velocidade relativamente lenta de $1 \mathrm{~cm} / \mathrm{s}$. Para permitir uma reconstrução da imagem dos tecidos subjacentes foi utilizado um software especializado (GE Medical Systems Lunar Software), permitindo a quantificação de tecidos ósseos e tecidos moles, como músculo e gordura. Todos os exames foram realizados pelo mesmo avaliador.

O dinamômetro de preensão manual (Handgrip; Model J00105; Jamar Hydraulic Hand Dynamometer, Sammons Preston Rolyan, Bolingbrook, Illionis, USA) foi utilizado para avaliar a força muscular dos pacientes. O teste foi realizado com a mão dominante na posição supinada e o cotovelo em 90 graus de flexão. O paciente realizou três tentativas com um intervalo de 1 minuto entre as mensurações e o maior valor foi utilizado para determinação da força muscular máxima ${ }^{84}$.

Artigo 1. Para definir a prevalência da sarcopenia, três índices foram utilizados: (1) massa muscular apendicular (ALM), caracterizada pela soma da massa muscular de ambos braços e pernas, dividida pela altura ao quadrado (ALM/altura $\left.{ }^{2}\right)^{85}$, (2) uma regressão linear utilizando massa muscular total como variável dependente com altura e massa gorda total como variável independente ${ }^{86}$, e (3) ALM dividida pelo índice de massa corporal (IMC) $)^{87}$. O valor de corte para sarcopenia foi definido no percentil 20 de cada índice descrito acima associado ao ponto de corte da força muscular pelo handgrip de acordo com a categoria do $\mathrm{IMC}^{88}$.

Artigo 2. A sarcopenia foi definida pela ALM dividida pela altura ao quadrado $<7,26 \mathrm{~kg} / \mathrm{m}^{2}\left(\mathrm{ALM} /\right.$ altura $\left.^{2}\right)$ e handgrip $<30 \mathrm{~kg}$, como proposto pelo European Working Group on Sarcopenia in Older People ${ }^{88}$. 


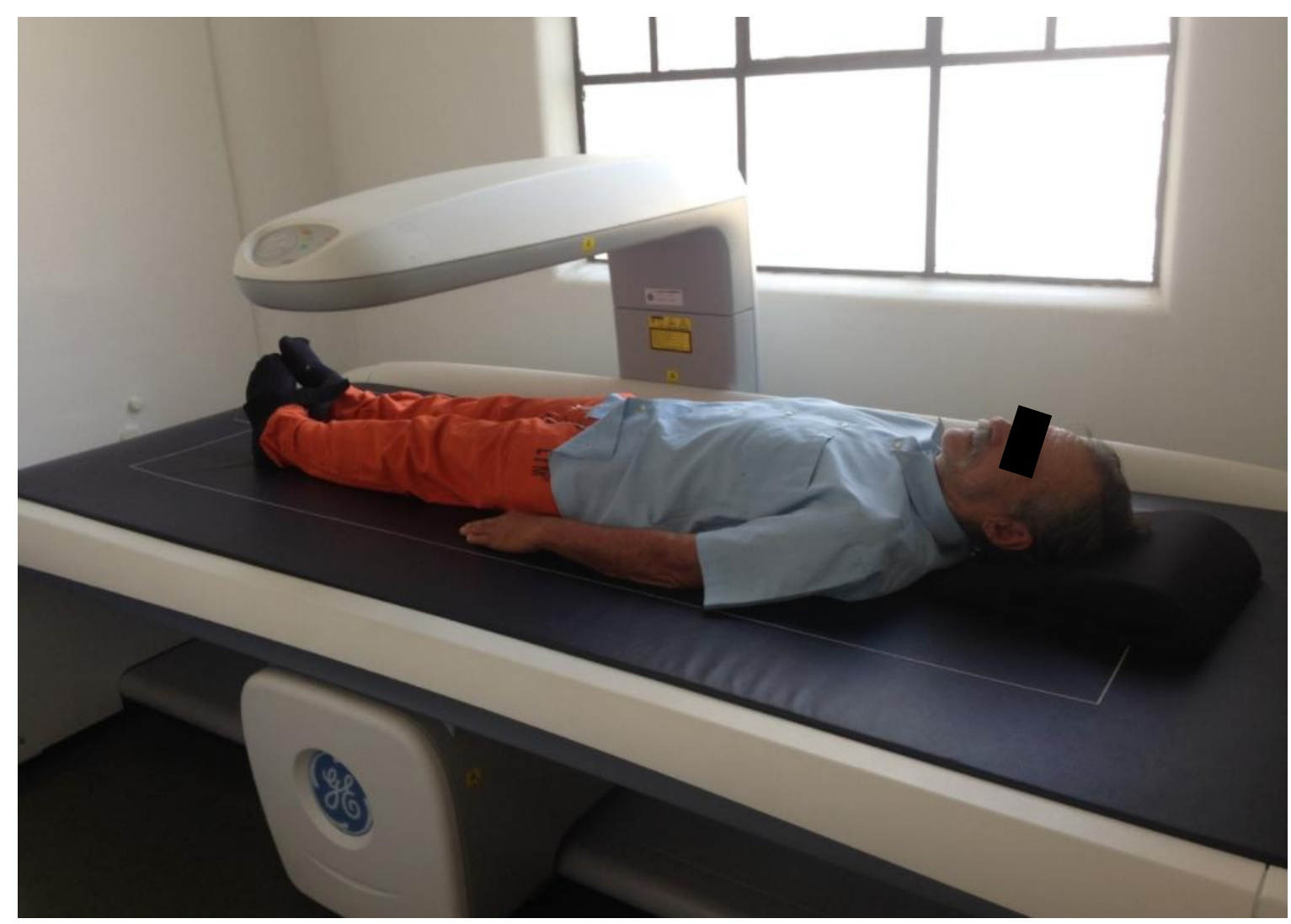

Figura 3. Densitometria computadorizada por absorciometria radiológica de dupla energia DXA.

\subsection{Avaliação da atividade nervosa simpática muscular}

Artigo 2. A MSNA foi avaliada através da técnica direta de microneurografia (Figura 4). Este procedimento registra a atividade de multiunidades da via pósgangliônica eferente do fascículo nervoso muscular do nervo fibular, localizado na perna, inferior à cabeça da fíbula. Os registros foram obtidos através da implantação de dois microeletrodos de tungstênio na perna direita do paciente, onde o primeiro foi posicionado na parte posterior do nervo fibular e o segundo, um microeletrodo referência, posicionado acima do primeiro aproximadamente a 2-3 centímetros de distância ${ }^{54}$. Esta técnica foi validada e empregada em estudos de laboratório em humanos ${ }^{89,90}$. Os eletrodos foram conectados a um pré-amplificador e o sinal do nervo alimentado através de um filtro passa-banda (700 a $2000 \mathrm{~Hz})$ que, em seguida, foi 
dirigido a um discriminador de amplitude para armazenagem em osciloscópio e em caixa de som. Para fins de registro e análise, o neurograma filtrado foi alimentado através de um integrador de capacitância-resistência para a obtenção da voltagem média da atividade neural.

A MSNA foi avaliada através de um registro contínuo da atividade simpática neuromuscular durante 10 minutos de repouso, com o paciente em decúbito dorsal. Durante esse período basal, um eletrocardiograma de cinco derivações foi realizado simultaneamente. $\mathrm{O}$ sinal do nervo foi analisado manualmente e contado minuto a minuto, por um único avaliador cego à condição hormonal dos pacientes. A MSNA foi expressa em disparos por minuto.

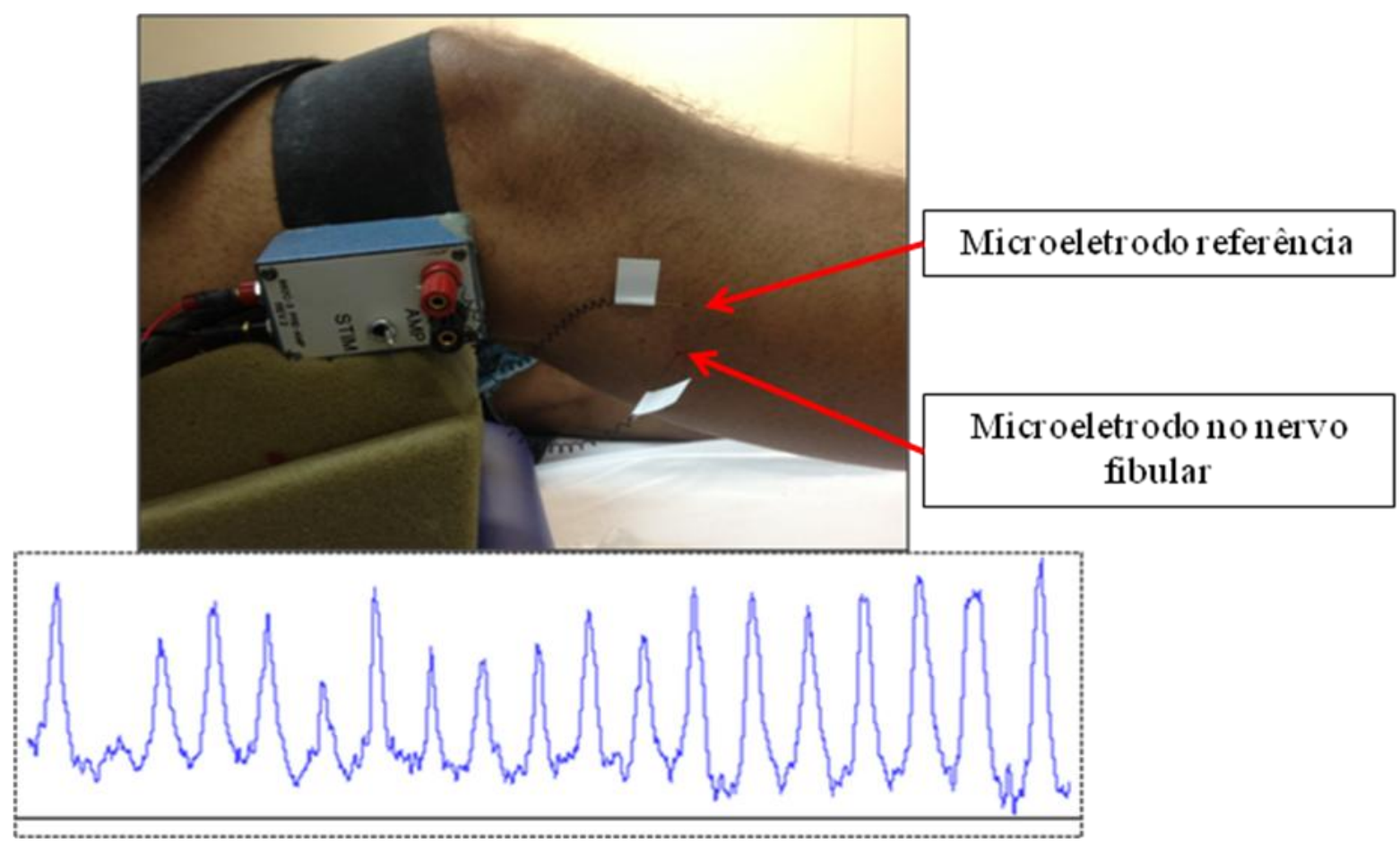

Figura 4. Avaliação da atividade nervosa simpática muscular (plano superior) e sinal de disparos espontâneos do nervo fibular (plano inferior) - Microneurografia. 


\subsection{Avaliação da reatividade parassimpática}

Artigo 2. A recuperação da frequência cardíaca (HRR) imediatamente após um teste de esforço físico máximo é um método indireto de mensurar a reatividade do sistema nervoso parassimpático. Dessa forma, o retardo na HRR possui valor prognóstico em pacientes com $\mathrm{IC}^{91}{ }^{92}$. Após atingir o esforço máximo no teste ergoespirométrico, os pacientes realizaram os primeiros 2 minutos de recuperação pedalando com $10 \mathrm{~W}$ e os 4 minutos seguintes sentados no cicloergômetro. $\mathrm{O}$ delta da HRR foi calculado pela subtração entre a FC máxima e a $\mathrm{FC}$ ao final do primeiro $(\Delta \mathrm{HRR} 1)$, e segundo minuto de recuperação $(\Delta \mathrm{HRR} 2)^{92}$.

\subsection{Avaliação do fluxo sanguíneo muscular do antebraço}

Artigo 3. Simultaneamente à realização da técnica de microneurografia, o fluxo sanguíneo muscular do antebraço (FBF) foi avaliado pela técnica de pletismografia de oclusão venosa (Figura 5). O braço não dominante foi elevado acima do nível do coração para garantir uma drenagem venosa adequada. Um tubo silástico preenchido com mercúrio, conectado a um transdutor de baixa pressão e ao pletismógrafo (Hokanson AI6, Bellevue, WA), foi colocado ao redor do antebraço a cinco centímetros de distância da articulação ulnoumeral. O aumento da tensão no tubo silástico reflete o aumento de volume do antebraço e, consequentemente, a medida de vasodilatação.

Os manguitos foram posicionados ao redor do punho e do braço. O manguito do punho foi inflado a uma pressão supra-sistólica $(200 \mathrm{mmHg}), 30$ segundos antes de iniciar as medidas e se manteve inflado no transcorrer do exame. Em intervalos de 20 segundos, o manguito do braço era inflado acima da pressão supra-venosa (60 mmHg) por um período de 10 segundos e desinflado por mais 10 segundos. $\mathrm{O}$ sinal de onda do 
FBF basal foi registrado em um polígrafo e analisado a cada minuto, realizando-se dessa forma três registros por minuto. A análise foi realizada manualmente por um único avaliador cego à condição hormonal dos pacientes.
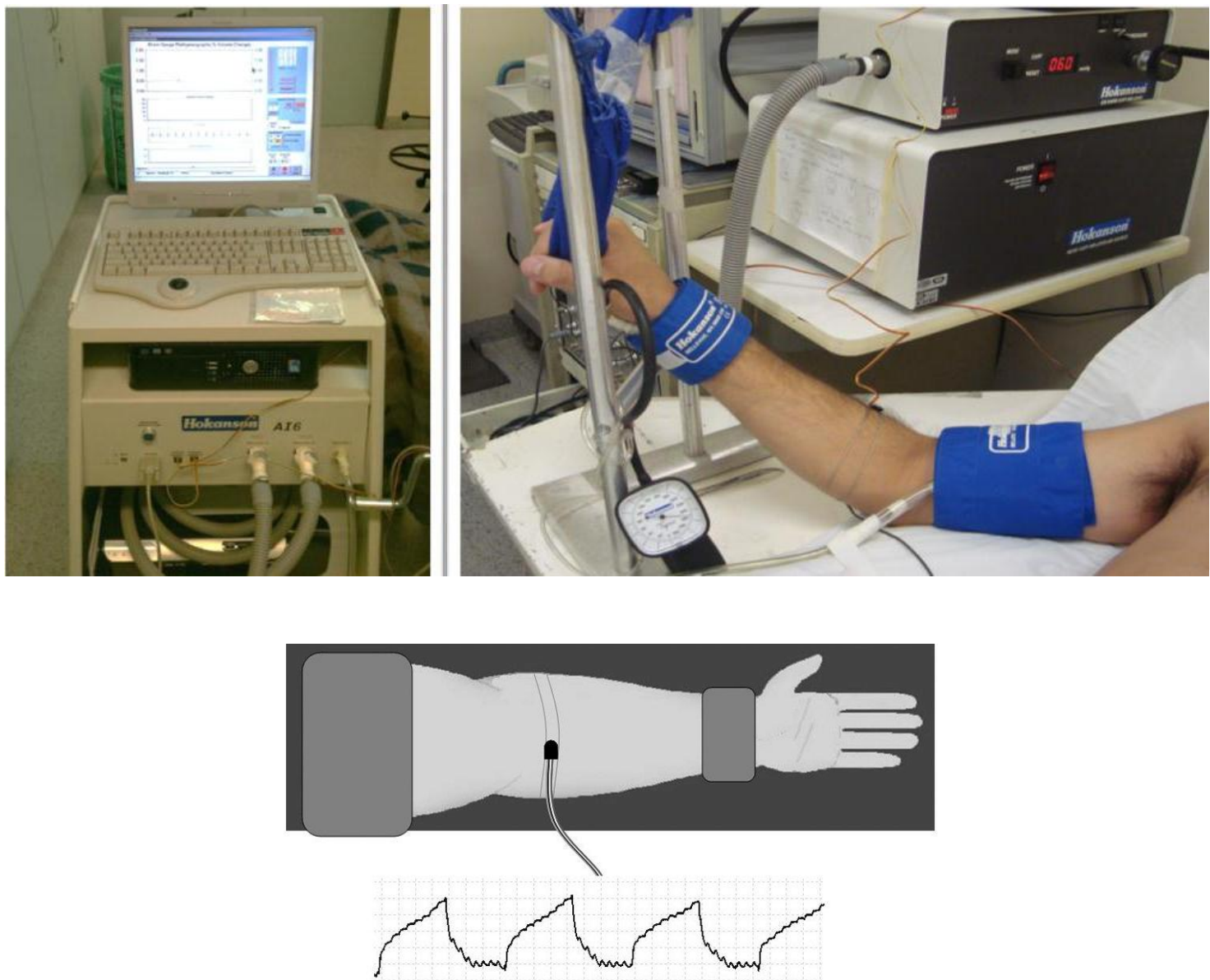

Figura 5. Avaliação do fluxo sanguíneo muscular do antebraço - Pletismografia de oclusão venosa. Ilustração adaptado de Casey, DP et al. (plano inferior) ${ }^{93}$.

O débito cardíaco, pressão arterial sistólica, diastólica e média foram avaliados pelo Finometer ${ }^{\circledR}$ PRO (Finapres Medical Systems), método não invasivo que avaliou a PA batimento a batimento (Figura 6). A FC foi obtida através do registro eletrocardiográfico de cinco derivações. Este sinal eletrocardiográfico foi préamplificado (General Purpose Amplifier/Stemtech, Inc., GPA-4, modelo 2) e convertido 
de analógico para digital, e em seguida, analisado em um programa de computador WINDAQ, numa frequência de $500 \mathrm{~Hz}$.

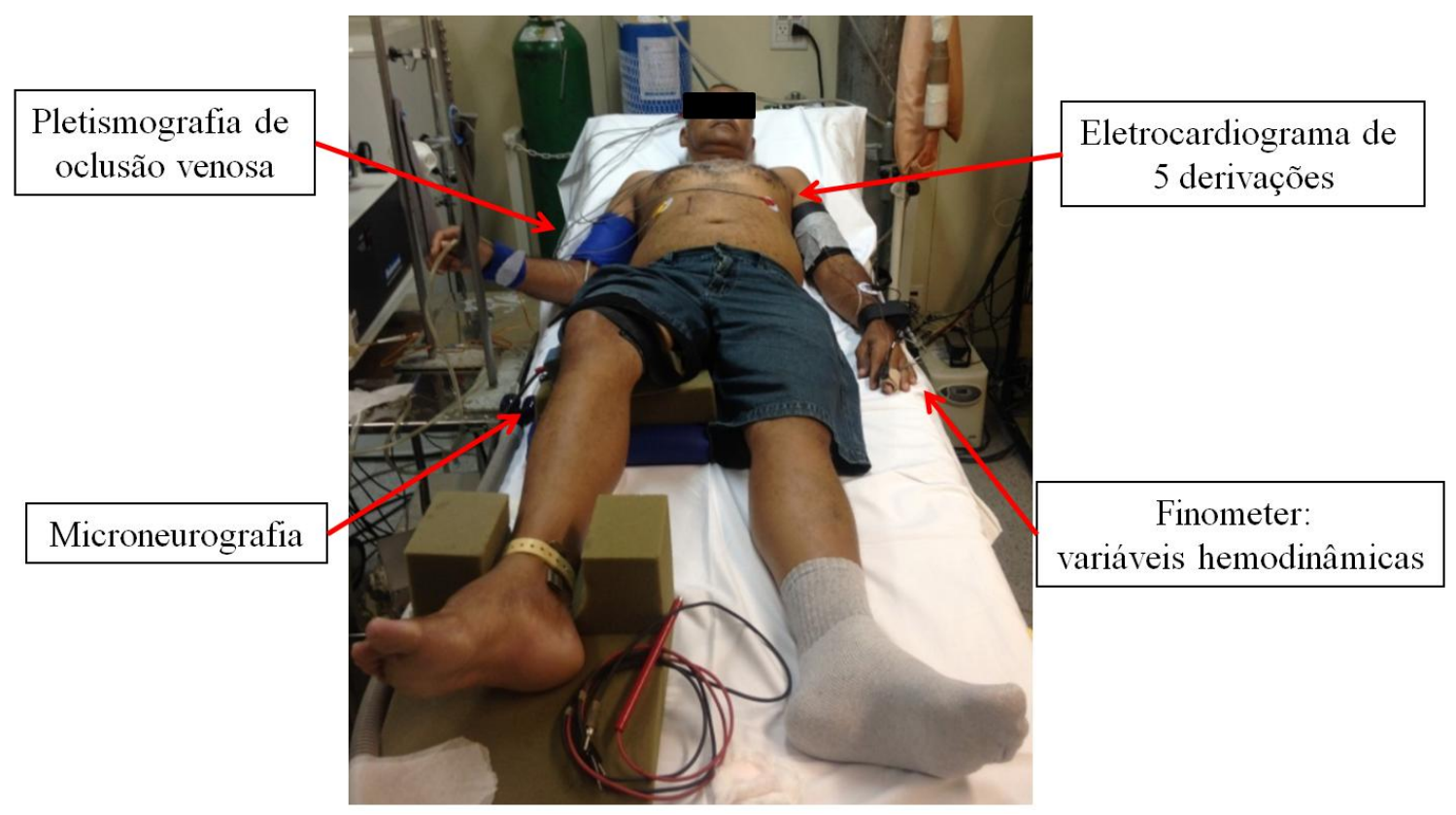

Figura 6. Posicionamento do paciente na realização da microneurografia, plestimografia de oclusão venosa, eletrocardiograma e variáveis hemodinâmicas. 


\section{ANÁLISE ESTATÍSTICA}

O risco proporcional de Cox com análise univariada e multivariada (stepwise backward para variáveis com $\mathrm{p} \leq 0,10$ ) foi utilizado para testar as associações entre as variáveis analisadas e o desfecho dos pacientes (mortalidade). A sobrevida dos pacientes foi avaliada pela análise de Kaplan-Meier com 95\% de intervalo de confiança (95\% CI) entre a concentração plasmática de TL e FBF para a incidência de eventos e mortalidade dentro do período de 36 meses. O teste de long-rank Cox-Mantel foi utilizado para determinar as diferenças nas taxas de sobrevida.

Artigo 1, 2 e 3. Os dados são apresentados como média \pm desvio padrão da média, mediana com intervalo interquartil $(95 \%$ CI) e frequência e percentual para variáveis categóricas. O teste de Kolmogorov-Smirnov foi utilizado para verificar todas as variáveis quanto à distribuição de normalidade. Para as variáveis categóricas, o teste Chi-square $\left(\mathrm{X}^{2}\right)$ foi utilizado para analisar a classe funcional, o tratamento medicamentoso e a etiologia da ICFEr. O programa Statistical Package for the Social Sciences version 23 (SPSS Inc., Chicago, Illinois, USA) foi utilizado para todas as análises estatísticas. $\mathrm{O}$ valor de $\mathrm{p}<0,05$ foi considerado estatisticamente significante.

Artigo 1. O teste $t$ de Student e o teste $U$ de Mann-Whitney foram utilizados para comparar as variáveis paramétricas e não paramétricas, respectivamente. Para as análises entre os três grupos sarcopênicos, a análise de variância de um caminho (ANOVA one-way), com análise de post-hoc de Scheffé em caso de diferença estatística, e o teste de Kruskal-Wallis foram utilizados para variáveis paramétricas e não paramétricas, respectivamente.

Artigo 2. Para as variáveis paramétricas e não paramétricas, foram utilizados o teste $t$ de Student e o teste $U$ de Mann-Whitney, respectivamente. A correlação de Spearman foi utilizado quando apropriado. 
Artigo 3. Para as análises entre as três etiologias, a ANOVA one-way, com análise de post-hoc de Scheffé em caso de diferença estatística, foi utilizada para as variáveis paramétricas, enquanto o teste de Kruskal-Wallis foi utilizado para comparar as diferenças de hs-CRP e FBF entre os grupos. A correlação de Pearson e a análise de regressão logística foram utilizadas quando apropriado. 


\section{RESULTADOS}

Na tabela 1 estão descritos os resultados relacionados ao desfecho primário do estudo. Na análise univariada do risco proporcional de Cox, FEVE (OR, 0,950; 95\% CI, 0,913-0,990; $\mathrm{p}=0,014)$, BNP (OR, 5,960; 95\% CI, 3,162-11,234; $\mathrm{p}<0,0001)$, creatinina (OR, 1,644; 95\% CI, 1,198-2,257; p=0,002), hs-CRP<2,79 mg/L (OR, 0,589; 95\% CI, 0,338-1,026; $\mathrm{p}=0,061), \mathrm{VO}_{2 \text { pico }}(\mathrm{OR}, 0,883 ; 95 \% \mathrm{CI}, 0,838-0,930 ; \mathrm{p}<0,0001)$, presença de anemia (OR, 2,047; 95\% CI, 1,175-3,566; $\mathrm{p}=0,001), \mathrm{TT}<300 \mathrm{ng} / \mathrm{dL}(\mathrm{OR}, 1,663 ; 95 \%$ CI, 0,946-2,925; p=0,077), TL<131 pmol/L (OR, 3,331; 95\% CI, 1,891-5,869; $\mathrm{p}<0,0001)$, MSNA (OR, 1,027; 95\% CI, 1,000-1,054; $\mathrm{p}=0,048)$ e FBF $(\mathrm{OR}, 0,341 ; 95 \%$ CI, $0,181-0,643 ; \mathrm{p}<0,001)$ foram associados com maior mortalidade em pacientes com ICFEr.

$\mathrm{Na}$ análise multivariada stepwise backward do risco proporcional de Cox, BNP (OR, 3,597; 95\% CI, 1,750-7,392; p<0,001), TL<131 pmol/L (OR, 2,097; 95\% CI, $1,009-4,357 ; \mathrm{p}=0,047)$ e $\mathrm{FBF}(\mathrm{OR}, 0,568 ; 95 \% \mathrm{CI}, 0,273-1,184 ; \mathrm{p}=0,031)$ foram preditores independentes de mortalidade, após ajuste para variáveis de confusão como idade, FEVE, NYHA, creatinina, hs-CRP $<2,79 \mathrm{mg} / \mathrm{L}, \mathrm{VO}_{2 \text { pico, }}$ VE/VCO2, presença de anemia, presença de sarcopenia, TT<300 ng/dL, DHEA-S, IGF-1 e MSNA.

Para análise de Kaplan Meier, os 169 pacientes com ICFEr foram subdivididos em quatro grupos de acordo com a concentração plasmática de TL e medida do FBF: (1) pacientes com TL>131 pmol/L e FBF>1,85 mL/min ${ }^{-1} / 100 \mathrm{~mL}^{-1}(\mathrm{n}=77)$, (2) pacientes com TL $>131 \mathrm{pmol} / \mathrm{L}$ e $\mathrm{FBF}<1,85 \mathrm{~mL} / \mathrm{min}^{-1} / 100 \mathrm{~mL}^{-1}(\mathrm{n}=59)$, (3) $\mathrm{TL}<131 \mathrm{pmol} / \mathrm{L}$ e FBF $>1,85 \mathrm{~mL} / \mathrm{min}^{-1} / 100 \mathrm{~mL}^{-1}(\mathrm{n}=11)$, e (4) $\mathrm{TL}<131 \mathrm{pmol} / \mathrm{L}$ e $\mathrm{FBF}<1,85 \mathrm{~mL} / \mathrm{min}^{-}$ $1 / 100 \mathrm{~mL}^{-1}(\mathrm{n}=22)$. A sobrevida acumulativa para esses grupos foi respectivamente de $78 \%$ (17 eventos), $73 \%$ (16 eventos), 55\% (5 eventos) e 36\% (14 eventos). O tempo mediano de sobrevida foi de 32,4 meses (95\% CI, 30,5-34,3), 31,3 meses (95\% CI, 
28,7-33,9), 23,5 meses (95\% CI, 14,8-32,3) e 21,2 meses (95\% CI, 15,1-27,2) para os respectivos grupos. O tempo de sobrevida foi estatisticamente diferente entre os grupos (log rank Mantel-Cox $\mathrm{p}<0,001 ;$ Figura 7). 
Tabela 1. Análise uni e multivariada do risco proporcional de Cox para mortalidade.

\begin{tabular}{|c|c|c|c|c|c|c|}
\hline \multirow[t]{2}{*}{ Variáveis } & \multicolumn{3}{|c|}{ Univariada } & \multicolumn{3}{|c|}{ Multivariada } \\
\hline & OR & $95 \% \mathrm{CI}$ & valor de $p$ & OR & $95 \% \mathrm{CI}$ & Valor de $\mathrm{p}$ \\
\hline $\begin{array}{lll}\text { Idade } & \text { (por } & 1 \\
\text { ano } & & \text { de } \\
\text { aumento) } & \end{array}$ & 0,997 & $\begin{array}{l}0,965- \\
1,031\end{array}$ & 0,871 & & & \\
\hline $\begin{array}{l}\text { FEVE (por } 1 \% \\
\text { de aumento) }\end{array}$ & 0,950 & $\begin{array}{l}0,913- \\
0,990\end{array}$ & 0,014 & 1,002 & $\begin{array}{c}0,958- \\
1,047\end{array}$ & 0,944 \\
\hline $\begin{array}{ll}\text { BNP (por } & 10 \\
\text { pg/mL } & \text { de } \\
\text { aumento) } & \end{array}$ & 5,960 & $\begin{array}{l}3,162- \\
11,234\end{array}$ & $<0,0001$ & 3,597 & $\begin{array}{l}1,750- \\
7,392\end{array}$ & $<0,001$ \\
\hline $\begin{array}{lr}\text { NYHA } & \text { (por } \\
\text { aumento } & \text { de } \\
\text { classe) } & \end{array}$ & 0,544 & $\begin{array}{l}0,239- \\
1,237\end{array}$ & 0,146 & & & \\
\hline $\begin{array}{l}\text { Creatinina (por } \\
1 \mathrm{mg} / \mathrm{dL} \text { de } \\
\text { aumento) }\end{array}$ & 1,644 & $\begin{array}{l}1,198- \\
2,257\end{array}$ & 0,002 & 1,201 & $\begin{array}{c}0,737- \\
1,956\end{array}$ & 0,462 \\
\hline $\begin{array}{l}\text { hs-CRP<2,79 } \\
\text { mg/L } \\
\text { (abaixo/acima } \\
\text { do corte) }\end{array}$ & 0,589 & $\begin{array}{l}0,338- \\
1,026\end{array}$ & 0,061 & 1,070 & $\begin{array}{l}0,573- \\
2,000\end{array}$ & 0,831 \\
\hline $\begin{array}{l}\mathrm{VO}_{2 \text { pico }} \text { (por } 1 \\
\mathrm{ml} / \mathrm{kg} / \mathrm{min} \text { de } \\
\text { aumento) }\end{array}$ & 0,883 & $\begin{array}{l}0,838- \\
0,930\end{array}$ & $<0,0001$ & 0,945 & $\begin{array}{c}0,888- \\
1,005\end{array}$ & 0,072 \\
\hline $\begin{array}{l}\mathrm{VE} / \mathrm{VCO}_{2} \text { (por } \\
1 \text { de aumento) }\end{array}$ & 1,024 & $\begin{array}{l}0,991- \\
1,057\end{array}$ & 0,152 & & & \\
\hline Presença de & 2,047 & $1,175-$ & 0,001 & 1,168 & $0,641-$ & 0,613 \\
\hline
\end{tabular}




\begin{tabular}{|c|c|c|c|c|c|c|}
\hline anemia & & 3,566 & & & 2,128 & \\
\hline $\begin{array}{ll}\text { Presença de } \\
\text { sarcopenia }\end{array}$ & 1,412 & $\begin{array}{l}0,797- \\
2,501\end{array}$ & 0,237 & & & \\
\hline $\begin{array}{l}\text { TT<300ng/dL } \\
\text { (abaixo/acima } \\
\text { do corte) }\end{array}$ & 1,663 & $\begin{array}{l}0,946- \\
2,925\end{array}$ & 0,077 & 0,730 & $\begin{array}{l}0,350- \\
1,523\end{array}$ & 0,401 \\
\hline $\begin{array}{l}\text { TL<131pmol/L } \\
\text { (abaixo/acima } \\
\text { do corte) }\end{array}$ & 3,331 & $\begin{array}{l}1,891- \\
5,869\end{array}$ & $<0,0001$ & 2,097 & $\begin{array}{l}1,009- \\
4,357\end{array}$ & 0,047 \\
\hline $\begin{array}{l}\text { DHEA-S } \\
\text { (ng/mL; } \\
\text { abaixo/acima } \\
\text { do corte para } \\
\text { idade) }\end{array}$ & 1,727 & $\begin{array}{l}0,888- \\
3,358\end{array}$ & 0,107 & & & \\
\hline $\begin{array}{l}\text { IGF-1 (ng/mL; } \\
\text { abaixo/acima } \\
\text { do corte para } \\
\text { idade) }\end{array}$ & 0,747 & $\begin{array}{r}0,384- \\
1,453\end{array}$ & 0,390 & & & \\
\hline $\begin{array}{l}\text { MSNA } \\
\text { (disparos/min; } \\
\text { por } 1 \text { disparo } \\
\text { de aumento) }\end{array}$ & 1,027 & $\begin{array}{l}1,000- \\
1,054\end{array}$ & 0,048 & 1,016 & $\begin{array}{l}0,989- \\
1,043\end{array}$ & 0,242 \\
\hline $\begin{array}{ll}\text { FBF } & \text { (por } \\
1 \mathrm{~mL} / \mathrm{min}^{-} & \\
1 / 100 \mathrm{~mL}^{-1} \text { de } \\
\text { aumento) }\end{array}$ & 0,341 & $\begin{array}{l}0,181- \\
0,643\end{array}$ & $<0,001$ & 0,568 & $\begin{array}{l}0,273- \\
1,184\end{array}$ & 0,031 \\
\hline
\end{tabular}

$\mathbf{B N P}=$ peptídeo natriurético cerebral; $\mathbf{C I}=$ intervalo de confiança; DHEA-S $=$ dehidroepiandrosterona sulfato; FBF = fluxo sanguíneo muscular do antebraço; FEVE = fração de ejeção do ventrículo esquerdo; hs-CRP = proteína C-reativa ultra sensível; IGF-1 = fator de crescimento semelhante à insulina tipo 1; MSNA = atividade nervosa simpática muscular; $\mathbf{N Y H A}=$ New York Heart Association $; \mathbf{O R}=$ razão de 
chance; $\mathbf{T L}=$ testosterona livre; $\mathbf{T T}=$ testosterona total; $\mathbf{V E} / \mathbf{V C O} \mathbf{C}_{2}=$ ventilação/produção de dióxido de carbono; $\mathbf{V O}_{2 \text { pico }}=$ consumo pico de oxigênio.

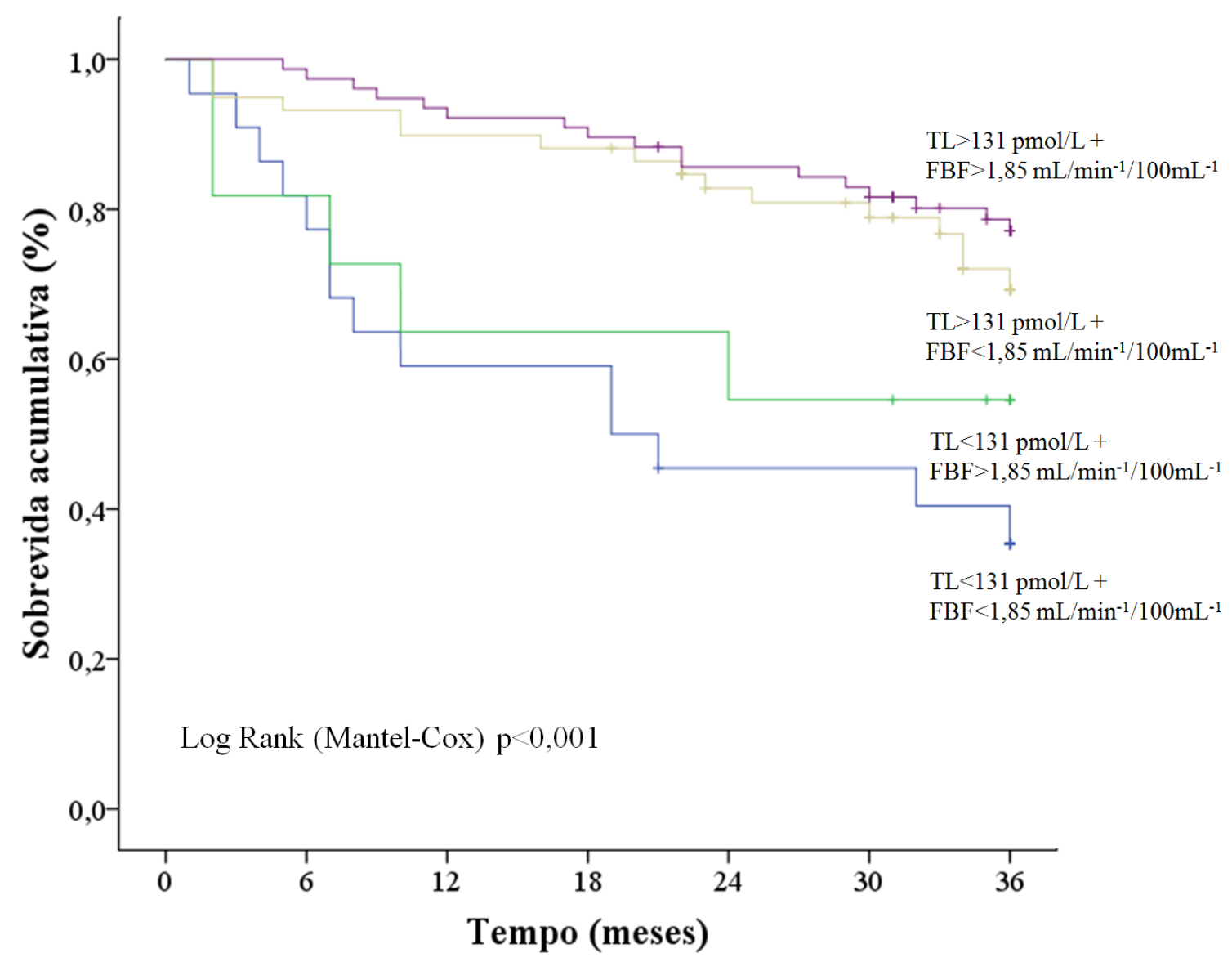

Figura 7. Curva de Kaplan Meier com TL (testosterona livre) e FBF (fluxo sanguíneo muscular do antebraço) para determinação de sobrevida acumulativa no período de acompanhamento de 36 meses. 


\section{Artigo 1}

Fonseca GWPD, Dos Santos MR, de Souza FR, Takayama L, Pereira RMR, Negrão CE, Alves MJNN. Discriminating sarcopenia in overweight/obese male patients with heart failure: the influence of body mass index. ESC Heart Fail. 2020 Feb; 7(1):84-91.

Nesse estudo, avaliamos a prevalência de sarcopenia em pacientes com ICFEr utilizando três métodos distintos de definição que corrigem a massa muscular pela altura ao quadrado (Índice de Baumgartner, mais utilizado na prática clínica), pela altura e massa gorda total como variáveis independentes em um regressão linear (Índice de Newman) ou pelo IMC (Índice de Studenski). Estas definições permitem uma abordagem ampla de todo o espectro da sarcopenia, que varia entre pacientes sarcopênicos com baixa e alta quantidade de gordura (obesidade sarcopênica). Os principais resultados desse estudo foram: 1) a prevalência de sarcopenia é similar entre pacientes "magros" $(20,8 \%)$ e "obesos" $(21,4 \%)$ quando índices que consideram a massa gorda são utilizados; 2) os índices que incluem a massa gorda na correção (Índices de Newman e Studenski) são mais sensíveis para detectar sarcopenia em pacientes com IMC $\geq 25 \mathrm{~kg} / \mathrm{m}^{2} ; 3$ ) embora os pacientes com obesidade sarcopênica tenha apresentado uma massa muscular maior que os pacientes sarcopênicos, a capacidade funcional foi reduzida em ambos os grupos em comparação com os pacientes sem sarcopenia (artigo em apêndice). 


\section{Artigo 2}

Fonseca GWPD, Dos Santos MR, de Souza FR, da Costa MJA, von Haehling S, Takayama L, Pereira RMR, Negrão CE, Anker SD, Alves MJNN. Sympatho-Vagal Imbalance is Associated with Sarcopenia in Male Patients with Heart Failure. Arq Bras Cardiol. 2019 Jun; 112(6):739-746.

Nesse estudo, avaliamos o impacto da modulação autonômica na sarcopenia em 116 pacientes com ICFEr. Os principais resultados desse estudo foram: 1) pacientes com sarcopenia apresentam maior MSNA do que pacientes sem sarcopenia [47 (41-52) vs. 40 (34-48) disparos/min; $\mathrm{p}=0,028$; respectivamente]; 2) pacientes sarcopênicos apresentam um menor $\Delta$ HRR1 [15 (10-21) vs. 22 (16-30) batimentos/min; p<0,001] e $\Delta$ HRR2 [25 (19-39) vs. 35 (24-48) batimentos/min, p=0,017] em comparação com pacientes não sarcopênicos, respectivamente; 3) houve uma correlação positiva entre ALM e $\triangle H R R 1(r=0,26 ; p=0,008)$ e $\Delta H R R 2(r=0,25 ; p=0,012)$, enquanto ALM se correlacionou negativamente com MSNA ( $\mathrm{r}=-0,29 ; \mathrm{p}=0,003)$ (artigo em apêndice). 


\section{Artigo 3 (artigo relacionado ao período de doutorado sanduíche)}

Fonseca GWPD, Macedo TG, Ebner N, Dos Santos MR, de Souza FR, Mady C, Takayama L, Pereira RMR, Doehner W, Anker SD, Negrão CE, Alves MJNN, von Haehling S. Muscle mass, muscle strength, and functional capacity in patients with heart failure of Chagas disease and other aetiologies. ESC Heart Fail. 2020 Aug 28.

Nesse estudo, avaliamos o impacto da etiologia sobre as alterações vasculares e de composição corporal em 64 pacientes interinstitucionais, subdivididos em 22 pacientes com doença de Chagas (TESTO-HF; InCor-Brasil), 20 pacientes com cardiomiopatia dilatada e 22 pacientes com miocardiopatia isquêmica (SICA-HF; University of Göttingen Medical Center-Alemanha). Os principais resultados desse estudo foram: 1) pacientes com ICFEr de origem chagásica e isquêmica apresentaram menor massa gorda $(16,3 \pm 8,1$ vs. $19,3 \pm 8,0$ vs. $27,6 \pm 9,4 \mathrm{~kg} ; \mathrm{p}<0,05)$ e $\mathrm{VO}_{2 \text { pico }}(1,17$ $\pm 0,36$ vs. $1,15 \pm 0,36$ vs. $1,50 \pm 0,45 \mathrm{~L} / \mathrm{min} ; \mathrm{p}<0,05)$ do que pacientes com cardiomiopatia dilatada, respectivamente; 2) pacientes chagásicos com ICFEr apresentaram menor força muscular $(27 \pm 8$ vs. $37 \pm 11$ vs. $36 \pm 14 \mathrm{~kg}$; p<0,05) e FBF $\left(1,84 \pm 0,54\right.$ vs. $2,75 \pm 0,76$ vs. $\left.3,42 \pm 1,21 \mathrm{~mL} / \mathrm{min}^{-1} / 100 \mathrm{~mL}^{-1} ; \mathrm{p}<0,01\right)$ em comparação com pacientes isquêmicos e com miocardiopatia dilatada, respectivamente; 3) houve uma correlação positiva entre $\operatorname{FBF}$ e $\operatorname{ALM}(r=0,25 ; p=0,046)$, massa muscular total $(\mathrm{r}=0,31 ; \mathrm{p}=0,012)$ e força muscular $(\mathrm{r}=0,36 ; \mathrm{p}=0,004) ; 4)$ ALM (OR, 1,179; $95 \% \mathrm{CI}$, 1,011-1,374; $\mathrm{p}=0,035)$ e hemoglobina (OR, 1,506; 95\% CI, 1,043-2,177; $\mathrm{p}=0,029$ ) foram independentemente associados com redução do $\mathrm{VO}_{2 \text { pico }}$ em pacientes com ICFEr, após ajuste para idade, FEVE, NYHA, creatinina e FBF (artigo em apêndice). 


\section{ANÁLISE CRÍTICA}

Os principais achados desse estudo foram: (1) o declínio na concentração plasmática de TL associado à redução do FBF são marcadores independentes de mortalidade em pacientes com ICFEr, (2) a prevalência de sarcopenia $(20,8 \%)$ e obesidade sarcopênica $(21,4 \%)$ são similares nessa população, quando índices que incluem massa gorda são utilizados, (3) pacientes sarcopênicos apresentam um desbalanço autonômico exacerbado, representado por um aumento de MSNA e menor reatividade vagal após teste de esforço máximo ( $\Delta \mathrm{HRR} 1$ e $\Delta \mathrm{HRR} 2)$, que é associado com a redução de ALM, e (4) pacientes de etiologia chagásica apresentam menor força muscular, FBF reduzido, menor massa gorda e uma tendência para redução de massa muscular em comparação com outras etiologias.

A deficiência anabólica hormonal é uma das diversas comorbidades que acomete pacientes com ICFEr, levando à um pior prognóstico, especialmente quando há o acúmulo de eixos anabólicos afetados ${ }^{18}$. Alguns estudos demonstram uma redução nas concentrações de TT e TL com um estímulo reflexo no aumento de LH e FSH nesses pacientes em comparação com indivíduos saudáveis pareados pela idade, sugerindo um quadro de hipogonadismo primário ${ }^{33}$. Em estudos populacionais analisando o impacto da redução na concentração plasmática de testosterona, ficou demonstrado que a TL parece ser mais sensível do que TT na determinação de mortalidade por todas as causas e por causas cardiovasculares em homens ${ }^{94,95}$. Nossos achados corroboram com esses trabalhos que evidenciam que a redução de TL é um marcador independente de mortalidade em pacientes com ICFEr, mesmo na presença de FBF menor ou maior que $1,85 \mathrm{~mL} / \mathrm{min}^{-1} / 100 \mathrm{~mL}^{-1}$, comparado com as outras duas condições, nas quais a TL é maior que 131 pmol/L (Figura 7; curva de Kaplan Meier). 
As alterações neurovasculares, como o aumento da MSNA e a redução do FBF, são também importantes marcadores de pior prognóstico em pacientes com ICFEr $^{19}$. Entretanto, apesar do aumento da MSNA estar intimamente relacionado com a progressão da ICFEr, nossos resultados demonstram que a redução do FBF tem um impacto mais significativo no desfecho de mortalidade nesses pacientes. A possível explicação para esse fenômeno parece ser devido aos avanços farmacológicos no tratamento da ICFEr, principalmente pelo uso de betabloqueadores (98\% dos pacientes no presente estudo), que aumentam a sobrevida dos pacientes por bloqueio da descarga simpática, central e periférico, e em contrapartida, há um aumento na participação de marcadores vasculares periféricos na progressão da doença.

A testosterona possui efeito sobre o leito vascular por meio do bloqueio nos receptores de cálcio do tipo-L e por um aumento no efluxo de potássio, promovendo assim um relaxamento das células musculares lisas ${ }^{96,97}$. A melhora da função endotelial atribuída à testosterona também pode ser intermediada por uma redução do perfil inflamatório e maior liberação de óxido nítrico ${ }^{46}$. Em pacientes com ICFEr, uma única administração de testosterona promoveu um aumento agudo de débito cardíaco acompanhado de redução na resistência vascular periférica ${ }^{98}$. Embora, a administração de testosterona em doses fisiológicas também apresente efeito adicional na melhora de capacidade funcional e força muscular em pacientes com $\mathrm{ICFEr}^{48}$, sua ação sistêmica em diversas tecidos, como a próstata, é passível de efeitos colaterais que devem ser levados em consideração na prescrição para essa população ${ }^{99}$.

A deficiência anabólica hormonal, assim como a redução do FBF por disfunção endotelial, são possíveis mecanismos também envolvidos no desenvolvimento de sarcopenia $^{65,72}$. Nosso primeiro artigo (apêndice) corroboram com outros estudos que demonstraram uma prevalência de sarcopenia em torno de $20 \%$ em pacientes com $\mathrm{IC}^{37}$. 
De forma complementar, este artigo também mostrou que a prevalência de obesidade sarcopênica, caracterizada pela associação da perda da massa muscular com a aumento no conteúdo de massa gorda, também apresentava similar prevalência à sarcopenia na ICFEr (21,4\% vs. 20,8\%). Até o presente momento, estudos envolvendo essa temática, sarcopenia associada à obesidade, demonstravam uma prevalência de até $10 \%$ em homens idosos e outras populações ${ }^{77,100-102}$. A prevalência aumentada de obesidade sarcopênica observada em nosso estudo pode passar a ser considerada também uma característica, até então desconhecida de pacientes com ICFEr, decorrente, em parte, da incidência elevada de sarcopenia e obesidade nessa população.

O desbalanço autonômico característico de pacientes com ICFEr pode ser outro mecanismo envolvido no desenvolvimento de sarcopenia nesses pacientes. Em estudo experimental ficou demonstrado que a hiperatividade simpática contribui para mudanças morfológicas e funcionais do músculo esquelético de ratos ${ }^{64}$. Nosso segundo artigo (apêndice) demonstrou que pacientes com ICFEr apresentavam um aumento da MSNA e redução da atividade parassimpática, avaliada pela reativação vagal após o teste de esforço máximo, que foi correlacionada com a diminuição da ALM. De maneira adicional, pacientes sarcopênicos, comparados aos não sarcopênicos, apresentavam uma redução do $\mathrm{VO}_{2 \text { pico, }} \mathrm{VE}$ e da carga máxima de trabalho (Watt) no teste ergoespirométrico.

A etiologia da ICFEr também pode influenciar as alterações de composição corporal e diminuição da capacidade funcional. Em pacientes com IC e doença de Chagas, uma redução de peso corporal foi observada em comparação com pacientes que apresentavam apenas doença de Chagas ${ }^{103}$, enquanto o IMC de pacientes com IC de etiologia chagásica foi menor do que pacientes de origem isquêmica e não isquêmica, sugerindo uma perda de composição corporal mais acentuada com a sobreposição da 
doença de Chagas e $\mathrm{IC}^{104}$. Nosso terceiro artigo (apêndice) sustenta essa hipótese demonstrando que pacientes chagásicos com ICFEr apresentavam uma massa gorda similar aos pacientes de origem isquêmica, mas com uma tendência para redução de massa muscular. Ademais, pacientes chagásicos apresentaram pior FBF, menor força muscular e massa óssea do que pacientes com cardiomiopatia dilatada e de origem isquêmica. Importante destacar que os pacientes com ICFEr e doença de Chagas eram mais jovens, evidenciando que essas alterações de composição corporal podem ser mais precoces em pacientes de etiologia chagásica, com uma perda de massa muscular mais significante com a progressão associadas dessas doenças. 


\section{CONCLUSÃO}

A redução da concentração plasmática de TL associada à diminuição de FBF mostrou ter valor prognóstico em pacientes com ICFEr. Estas alterações estão relacionadas com a progressão da doença e podem levar ao quadro de sarcopenia ou obesidade sarcopênica, que são comorbidades distintas, mas apresentam uma prevalência muito semelhante, em torno de 20\%. O desbalanço autonômico exacerbado, evidenciado pelo aumento da MSNA e redução da reatividade vagal após o teste de esforço máximo em pacientes sarcopênicos, pode ser um dos mecanismos envolvidos no desenvolvimento de sarcopenia em pacientes com ICFEr. Ademais, pacientes de etiologia chagásica apresentam piora no FBF, menor força muscular e redução de massa gorda com uma tendência de diminuição da massa muscular, de forma mais precoce, em comparação com as etiologias isquêmica e não isquêmica. 


\section{REFERÊNCIAS BIBLIOGRÁFICAS}

1. Tanai E, Frantz S. Pathophysiology of Heart Failure. Compr Physiol. 2015;6(1):187-214.

2. Ponikowski P, Voors AA, Anker SD, Bueno H, Cleland JGF, Coats AJS, et al. 2016 ESC Guidelines for the Diagnosis and Treatment of Acute and Chronic Heart Failure. Rev Esp Cardiol (Engl Ed). 2016;69(12):1167.

3. Rohde LEP, Montera MW, Bocchi EA, Clausell NO, Albuquerque DC, Rassi S, et al. Diretriz Brasileira de Insuficiência Cardíaca Crônica e Aguda. Arq Bras Cardiol. 2018;111(3):436-539.

4. Mosterd A, Hoes AW. Clinical epidemiology of heart failure. Heart. 2007;93(9):1137-46.

5. Bleumink GS, Knetsch AM, Sturkenboom MC, Straus SM, Hofman A, Deckers JW, et al. Quantifying the heart failure epidemic: prevalence, incidence rate, lifetime risk and prognosis of heart failure The Rotterdam Study. Eur Heart J. 2004;25(18):1614-9.

6. McMurray JJ, Pfeffer MA. Heart failure. Lancet. 2005;365(9474):1877-89.

7. Levy D, Kenchaiah S, Larson MG, Benjamin EJ, Kupka MJ, Ho KK, et al. Long-term trends in the incidence of and survival with heart failure. $N$ Engl J Med. 2002;347(18):1397-402. 
8. Gerber Y, Weston SA, Redfield MM, Chamberlain AM, Manemann SM, Jiang

$\mathrm{R}$, et al. A contemporary appraisal of the heart failure epidemic in Olmsted County, Minnesota, 2000 to 2010. JAMA Intern Med. 2015;175(6):996-1004.

9. Mendez GF, Cowie MR. The epidemiological features of heart failure in developing countries: a review of the literature. Int J Cardiol. 2001;80(2-3):213-9.

10. Keith M, Geranmayegan A, Sole MJ, Kurian R, Robinson A, Omran AS, et al. Increased oxidative stress in patients with congestive heart failure. J Am Coll Cardiol. 1998;31(6):1352-6.

11. Cubbon RM, Adams B, Rajwani A, Mercer BN, Patel PA, Gherardi G, et al. Diabetes mellitus is associated with adverse prognosis in chronic heart failure of ischaemic and non-ischaemic aetiology. Diab Vasc Dis Res. 2013;10(4):330-6.

12. Sakatani T, Shirayama T, Suzaki Y, Yamamoto T, Mani H, Kawasaki T, et al. The association between cholesterol and mortality in heart failure. Comparison between patients with and without coronary artery disease. Int Heart J. 2005;46(4):619-29.

13. Kenchaiah S, Evans JC, Levy D, Wilson PW, Benjamin EJ, Larson MG, et al. Obesity and the risk of heart failure. N Engl J Med. 2002;347(5):305-13.

14. Abebe TB, Gebreyohannes EA, Bhagavathula AS, Tefera YG, Abegaz TM. Anemia in severe heart failure patients: does it predict prognosis? BMC Cardiovasc Disord. 2017;17(1):248. 
15. Damman K, Valente MA, Voors AA, O'Connor CM, van Veldhuisen DJ, Hillege HL. Renal impairment, worsening renal function, and outcome in patients with heart failure: an updated meta-analysis. Eur Heart J. 2014;35(7):455-69.

16. Pearse SG, Cowie MR. Sleep-disordered breathing in heart failure. Eur J Heart Fail. 2016;18(4):353-61.

17. Plesner LL, Dalsgaard M, Schou M, Kober L, Vestbo J, Kjoller E, et al. The prognostic significance of lung function in stable heart failure outpatients. Clin Cardiol. 2017;40(11):1145-51.

18. Jankowska EA, Biel B, Majda J, Szklarska A, Lopuszanska M, Medras M, et al. Anabolic deficiency in men with chronic heart failure: prevalence and detrimental impact on survival. Circulation. 2006;114(17):1829-37.

19. Barretto AC, Santos AC, Munhoz R, Rondon MU, Franco FG, Trombetta IC, et al. Increased muscle sympathetic nerve activity predicts mortality in heart failure patients. Int J Cardiol. 2009;135(3):302-7.

20. Forman DE, Santanasto AJ, Boudreau R, Harris T, Kanaya AM, Satterfield S, et al. Impact of Incident Heart Failure on Body Composition Over Time in the Health, Aging, and Body Composition Study Population. Circ Heart Fail. 2017;10(9):e003915.

21. Iervasi G, Pingitore A, Landi P, Raciti M, Ripoli A, Scarlattini M, et al. Low-T3 syndrome: a strong prognostic predictor of death in patients with heart disease. Circulation. 2003;107(5):708-13. 
22. Sacca L. Heart failure as a multiple hormonal deficiency syndrome. Circ Heart Fail. 2009;2(2):151-6.

23. Brenta G, Thierer J, Sutton M, Acosta A, Vainstein N, Brites F, et al. Low plasma triiodothyronine levels in heart failure are associated with a reduced anabolic state and membrane damage. Eur J Endocrinol. 2011;164(6):937-42.

24. Opasich C, Pacini F, Ambrosino N, Riccardi PG, Febo O, Ferrari R, et al. Sick euthyroid syndrome in patients with moderate-to-severe chronic heart failure. Eur Heart J. 1996;17(12):1860-6.

25. Ascheim DD, Hryniewicz K. Thyroid hormone metabolism in patients with congestive heart failure: the low triiodothyronine state. Thyroid. 2002;12(6):511-5.

26. Yamaji M, Tsutamoto T, Kawahara C, Nishiyama K, Yamamoto T, Fujii M, et al. Serum cortisol as a useful predictor of cardiac events in patients with chronic heart failure: the impact of oxidative stress. Circ Heart Fail. 2009;2(6):608-15.

27. Swan JW, Anker SD, Walton C, Godsland IF, Clark AL, Leyva F, et al. Insulin resistance in chronic heart failure: relation to severity and etiology of heart failure. $J \mathrm{Am}$ Coll Cardiol. 1997;30(2):527-32.

28. Kontoleon PE, Anastasiou-Nana MI, Papapetrou PD, Alexopoulos G, Ktenas V, Rapti AC, et al. Hormonal profile in patients with congestive heart failure. Int $J$ Cardiol. 2003;87(2-3):179-83. 
29. Anker SD, Chua TP, Ponikowski P, Harrington D, Swan JW, Kox WJ, et al. Hormonal changes and catabolic/anabolic imbalance in chronic heart failure and their importance for cardiac cachexia. Circulation. 1997;96(2):526-34.

30. Josiak K, Jankowska EA, Piepoli MF, Banasiak W, Ponikowski P. Skeletal myopathy in patients with chronic heart failure: significance of anabolic-androgenic hormones. J Cachexia Sarcopenia Muscle. 2014;5(4):287-96.

31. Basaria S. Male hypogonadism. Lancet. 2014;383(9924):1250-63.

32. Marsh JD, Lehmann MH, Ritchie RH, Gwathmey JK, Green GE, Schiebinger RJ. Androgen receptors mediate hypertrophy in cardiac myocytes. Circulation. 1998;98(3):256-61.

33. Chen Q, Li XY, Li JY, Zhang WY, Li HT, Liu F. [A comparative survey of sex hormones levels between elderly men with chronic heart failure and normal elderly subjects]. Zhonghua Xin Xue Guan Bing Za Zhi. 2005;33(6):505-8.

34. Moriyama Y, Yasue H, Yoshimura M, Mizuno Y, Nishiyama K, Tsunoda R, et al. The plasma levels of dehydroepiandrosterone sulfate are decreased in patients with chronic heart failure in proportion to the severity. $J$ Clin Endocrinol Metab. 2000;85(5):1834-40.

35. Wu FC, Tajar A, Beynon JM, Pye SR, Silman AJ, Finn JD, et al. Identification of late-onset hypogonadism in middle-aged and elderly men. $N$ Engl J Med. 2010;363(2):123-35. 
36. Pastor-Perez FJ, Manzano-Fernandez S, Garrido Bravo IP, Nicolas F, Tornel PL, Lax A, et al. Anabolic status and functional impairment in men with mild chronic heart failure. Am J Cardiol. 2011;108(6):862-6.

37. Fulster S, Tacke M, Sandek A, Ebner N, Tschope C, Doehner W, et al. Muscle wasting in patients with chronic heart failure: results from the studies investigating comorbidities aggravating heart failure (SICA-HF). Eur Heart J. 2013;34(7):512-9.

38. Xiong Y, Hales DB. The role of tumor necrosis factor-alpha in the regulation of mouse Leydig cell steroidogenesis. Endocrinology. 1993;132(6):2438-44.

39. Jankowska EA, Filippatos G, Ponikowska B, Borodulin-Nadzieja L, Anker SD, Banasiak W, et al. Reduction in circulating testosterone relates to exercise capacity in men with chronic heart failure. J Card Fail. 2009;15(5):442-50.

40. Han Y, Sun W, Sun G, Hou X, Gong Z, Xu J, et al. A 3-year observation of testosterone deficiency in Chinese patients with chronic heart failure. Oncotarget. 2017;8(45):79835-42.

41. Santos MR, Sayegh AL, Groehs RV, Fonseca G, Trombetta IC, Barretto AC, et al. Testosterone deficiency increases hospital readmission and mortality rates in male patients with heart failure. Arq Bras Cardiol. 2015;105(3):256-64.

42. Wehr E, Pilz S, Boehm BO, Marz W, Grammer T, Obermayer-Pietsch B. Low free testosterone is associated with heart failure mortality in older men referred for coronary angiography. Eur J Heart Fail. 2011;13(5):482-8. 
43. Pascual-Figal DA, Tornel PL, Nicolas F, Sanchez-Mas J, Martinez MD, Gracia MR, et al. Sex hormone-binding globulin: a new marker of disease severity and prognosis in men with chronic heart failure. Rev Esp Cardiol. 2009;62(12):1381-7.

44. Wu HY, Wang XF, Wang JH, Li JY. Testosterone level and mortality in elderly men with systolic chronic heart failure. Asian J Androl. 2011;13(5):759-63.

45. Guder G, Frantz S, Bauersachs J, Allolio B, Ertl G, Angermann CE, et al. Low circulating androgens and mortality risk in heart failure. Heart. 2010;96(7):504-9.

46. Kelly DM, Jones TH. Testosterone: a vascular hormone in health and disease. $J$ Endocrinol. 2013;217(3):R47-71.

47. Jones TH, Kelly DM. Randomized controlled trials - mechanistic studies of testosterone and the cardiovascular system. Asian J Androl. 2018;20(2):120-30.

48. Caminiti G, Volterrani M, Iellamo F, Marazzi G, Massaro R, Miceli M, et al. Effect of long-acting testosterone treatment on functional exercise capacity, skeletal muscle performance, insulin resistance, and baroreflex sensitivity in elderly patients with chronic heart failure a double-blind, placebo-controlled, randomized study. $J$ Am Coll Cardiol. 2009;54(10):919-27.

49. Wang Y, Seto SW, Golledge J. Angiotensin II, sympathetic nerve activity and chronic heart failure. Heart Fail Rev. 2014;19(2):187-98. 
50. Notarius CF, Millar PJ, Floras JS. Muscle sympathetic activity in resting and exercising humans with and without heart failure. Appl Physiol Nutr Metab. 2015;40(11):1107-15.

51. Negrao CE, Middlekauff HR, Gomes-Santos IL, Antunes-Correa LM. Effects of exercise training on neurovascular control and skeletal myopathy in systolic heart failure. Am J Physiol Heart Circ Physiol. 2015;308(8):H792-802.

52. Cohn JN, Levine TB, Olivari MT, Garberg V, Lura D, Francis GS, et al. Plasma norepinephrine as a guide to prognosis in patients with chronic congestive heart failure. N Engl J Med. 1984;311(13):819-23.

53. Seals DR, Esler MD. Human ageing and the sympathoadrenal system. J Physiol. 2000;528(3):407-17.

54. Vallbo AB, Hagbarth KE, Wallin BG. Microneurography: how the technique developed and its role in the investigation of the sympathetic nervous system. $J$ Appl Physiol (1985). 2004;96(4):1262-9.

55. Grassi G, Bolla G, Quarti-Trevano F, Arenare F, Brambilla G, Mancia G. Sympathetic activation in congestive heart failure: reproducibility of neuroadrenergic markers. Eur J Heart Fail. 2008;10(12):1186-91.

56. Leimbach WN, Jr., Wallin BG, Victor RG, Aylward PE, Sundlof G, Mark AL. Direct evidence from intraneural recordings for increased central sympathetic outflow in patients with heart failure. Circulation. 1986;73(5):913-9. 
57. Ferguson DW, Berg WJ, Sanders JS. Clinical and hemodynamic correlates of sympathetic nerve activity in normal humans and patients with heart failure: evidence from direct microneurographic recordings. J Am Coll Cardiol. 1990;16(5):1125-34.

58. Munhoz RT, Negrao CE, Barretto AC, Ochiai ME, Cardoso JN, Morgado PC, et al. Microneurography and venous occlusion plethysmography in heart failure: correlation with prognosis. Arq Bras Cardiol. 2009;92(1):46-53.

59. Alves MJ, Rondon MU, Santos AC, Dias RG, Barretto AC, Krieger EM, et al. Sympathetic nerve activity restrains reflex vasodilatation in heart failure. Clin Auton Res. 2007;17(6):364-9.

60. Santos AC, Alves MJ, Rondon MU, Barretto AC, Middlekauff HR, Negrao CE. Sympathetic activation restrains endothelium-mediated muscle vasodilatation in heart failure patients. Am J Physiol Heart Circ Physiol. 2005;289(2):H593-9.

61. Nazare Nunes Alves MJ, dos Santos MR, Nobre TS, Martinez DG, Pereira Barretto AC, Brum PC, et al. Mechanisms of blunted muscle vasodilation during peripheral chemoreceptor stimulation in heart failure patients. Hypertension. 2012;60(3):669-76.

62. Notarius CF, Ando S, Rongen GA, Floras JS. Resting muscle sympathetic nerve activity and peak oxygen uptake in heart failure and normal subjects. Eur Heart J. 1999;20(12):880-7. 
63. Notarius CF, Millar PJ, Murai H, Morris BL, Marzolini S, Oh P, et al. Divergent muscle sympathetic responses to dynamic leg exercise in heart failure and age-matched healthy subjects. J Physiol. 2015;593(3):715-22.

64. Bacurau AV, Jardim MA, Ferreira JC, Bechara LR, Bueno CR, Jr., AlbaLoureiro TC, et al. Sympathetic hyperactivity differentially affects skeletal muscle mass in developing heart failure: role of exercise training. J Appl Physiol (1985). 2009;106(5):1631-40.

65. Dos Santos MR, Saitoh M, Ebner N, Valentova M, Konishi M, Ishida J, et al. Sarcopenia and Endothelial Function in Patients With Chronic Heart Failure: Results From the Studies Investigating Comorbidities Aggravating Heart Failure (SICA-HF). $J$ Am Med Dir Assoc. 2017;18(3):240-5.

66. Cruz-Jentoft AJ, Bahat G, Bauer J, Boirie Y, Bruyère O, Cederholm T, et al. Sarcopenia: revised European consensus on definition and diagnosis. Age Ageing. 2019;48(1):16-31.

67. Rosenberg IH. Sarcopenia: origins and clinical relevance. $J$ Nutr. 1997;127(5):990S-1S.

68. Hajahmadi M, Shemshadi S, Khalilipur E, Amin A, Taghavi S, Maleki M, et al. Muscle wasting in young patients with dilated cardiomyopathy. J Cachexia Sarcopenia Muscle. 2017;8(4):542-8. 
69. Marzetti E, Calvani R, Cesari M, Buford TW, Lorenzi M, Behnke BJ, et al. Mitochondrial dysfunction and sarcopenia of aging: from signaling pathways to clinical trials. Int J Biochem Cell Biol. 2013;45(10):2288-301.

70. Degens $\mathrm{H}$. The role of systemic inflammation in age-related muscle weakness and wasting. Scand J Med Sci Sports. 2010;20(1):28-38.

71. Drey M, Krieger B, Sieber CC, Bauer JM, Hettwer S, Bertsch T, et al. Motoneuron loss is associated with sarcopenia. J Am Med Dir Assoc. 2014;15(6):435-9.

72. Morley JE. Hormones and Sarcopenia. Curr Pharm Des. 2017;23(30):4484-92.

73. Fonseca H, Powers SK, Gonçalves D, Santos A, Mota MP, Duarte JA. Physical inactivity is a major contributor to ovariectomy-induced sarcopenia. Int J Sports Med. 2012;33(4):268-78.

74. Onishi S, Shiraki M, Nishimura K, Hanai T, Moriwaki H, Shimizu M. Prevalence of Sarcopenia and Its Relationship with Nutritional State and Quality of Life in Patients with Digestive Diseases. J Nutr Sci Vitaminol (Tokyo). 2018;64(6):445-53.

75. Florea VG, Cohn JN. The autonomic nervous system and heart failure. Circ Res. 2014;114(11):1815-26.

76. Batsis JA, Villareal DT. Sarcopenic obesity in older adults: aetiology, epidemiology and treatment strategies. Nat Rev Endocrinol. 2018;14(9):513-37. 
77. Kim TN, Yang SJ, Yoo HJ, Lim KI, Kang HJ, Song W, et al. Prevalence of sarcopenia and sarcopenic obesity in Korean adults: the Korean sarcopenic obesity study. Int J Obes (Lond). 2009;33(8):885-92.

78. Tian $\mathrm{S}, \mathrm{Xu} \mathrm{Y}$. Association of sarcopenic obesity with the risk of all-cause mortality: A meta-analysis of prospective cohort studies. Geriatr Gerontol Int. 2016;16(2):155-66.

79. Vermeulen A, Verdonck L, Kaufman JM. A critical evaluation of simple methods for the estimation of free testosterone in serum. J Clin Endocrinol Metab. 1999;84(10):3666-72.

80. Mulhall JP, Trost LW, Brannigan RE, Kurtz EG, Redmon JB, Chiles KA, et al. Evaluation and Management of Testosterone Deficiency: AUA Guideline. J Urol. 2018;200(2):423-32.

81. Huhtaniemi IT, Tajar A, Lee DM, O'Neill TW, Finn JD, Bartfai G, et al. Comparison of serum testosterone and estradiol measurements in 3174 European men using platform immunoassay and mass spectrometry; relevance for the diagnostics in aging men. Eur J Endocrinol. 2012;166(6):983-91.

82. Wasserman K, Whipp BJ. Excercise physiology in health and disease. Am Rev Respir Dis. 1975;112(2):219-49.

83. Skinner JS, McLellan TM, McLellan TH. The transition from aerobic to anaerobic metabolism. Res Q Exerc Sport. 1980;51(1):234-48. 
84. Roberts HC, Denison HJ, Martin HJ, Patel HP, Syddall H, Cooper C, et al. A review of the measurement of grip strength in clinical and epidemiological studies: towards a standardised approach. Age Ageing. 2011;40(4):423-9.

85. Baumgartner RN, Koehler KM, Gallagher D, Romero L, Heymsfield SB, Ross RR, et al. Epidemiology of sarcopenia among the elderly in New Mexico. Am $J$ Epidemiol. 1998;147(8):755-63.

86. Newman AB, Kupelian V, Visser M, Simonsick E, Goodpaster B, Nevitt M, et al. Sarcopenia: alternative definitions and associations with lower extremity function. $J$ Am Geriatr Soc. 2003;51(11):1602-9.

87. Studenski SA, Peters KW, Alley DE, Cawthon PM, McLean RR, Harris TB, et al. The FNIH sarcopenia project: rationale, study description, conference recommendations, and final estimates. J Gerontol A Biol Sci Med Sci. 2014;69(5):54758.

88. Cruz-Jentoft AJ, Baeyens JP, Bauer JM, Boirie Y, Cederholm T, Landi F, et al. Sarcopenia: European consensus on definition and diagnosis: Report of the European Working Group on Sarcopenia in Older People. Age Ageing. 2010;39(4):412-23.

89. Vallbo AB, Hagbarth KE, Torebjork HE, Wallin BG. Somatosensory, proprioceptive, and sympathetic activity in human peripheral nerves. Physiol Rev. 1979;59(4):919-57. 
90. Fagius J, Wallin BG. Long-term variability and reproducibility of resting human muscle nerve sympathetic activity at rest, as reassessed after a decade. Clin Auton Res. 1993;3(3):201-5.

91. Myers J, Hadley D, Oswald U, Bruner K, Kottman W, Hsu L, et al. Effects of exercise training on heart rate recovery in patients with chronic heart failure. Am Heart J. 2007;153(6):1056-63.

92. Nanas S, Anastasiou-Nana M, Dimopoulos S, Sakellariou D, Alexopoulos G, Kapsimalakou S, et al. Early heart rate recovery after exercise predicts mortality in patients with chronic heart failure. Int J Cardiol. 2006;110(3):393-400.

93. Casey DP, Curry TB, Joyner MJ. Measuring muscle blood flow: a key link between systemic and regional metabolism. Curr Opin Clin Nutr Metab Care. 2008;11(5):580-6.

94. Vikan T, Schirmer H, Njølstad I, Svartberg J. Endogenous sex hormones and the prospective association with cardiovascular disease and mortality in men: the Troms $\varnothing$ Study. Eur J Endocrinol. 2009;161(3):435-42.

95. Hyde Z, Norman PE, Flicker L, Hankey GJ, Almeida OP, McCaul KA, et al. Low free testosterone predicts mortality from cardiovascular disease but not other causes: the Health in Men Study. J Clin Endocrinol Metab. 2012;97(1):179-89.

96. Scragg JL, Jones RD, Channer KS, Jones TH, Peers C. Testosterone is a potent inhibitor of L-type Ca(2+) channels. Biochem Biophys Res Commun. 2004;318(2):5036. 
97. Malkin CJ, Jones RD, Jones TH, Channer KS. Effect of testosterone on ex vivo vascular reactivity in man. Clin Sci (Lond). 2006;111(4):265-74.

98. Pugh PJ, Jones TH, Channer KS. Acute haemodynamic effects of testosterone in men with chronic heart failure. Eur Heart J. 2003;24(10):909-15.

99. Holmäng S, Mårin $\mathrm{P}$, Lindstedt $\mathrm{G}$, Hedelin $\mathrm{H}$. Effect of long-term oral testosterone undecanoate treatment on prostate volume and serum prostate-specific antigen concentration in eugonadal middle-aged men. Prostate. 1993;23(2):99-106.

100. Kemmler W, Teschler M, Weißenfels A, Sieber C, Freiberger E, von Stengel S. Prevalence of sarcopenia and sarcopenic obesity in older German men using recognized definitions: high accordance but low overlap! Osteoporos Int. 2017;28(6):1881-91.

101. Tabibi H, As'habi A, Najafi I, Hedayati M. Prevalence of dynapenic obesity and sarcopenic obesity and their associations with cardiovascular disease risk factors in peritoneal dialysis patients. Kidney Res Clin Pract. 2018;37(4):404-13.

102. Godziuk K, Prado CM, Woodhouse LJ, Forhan M. Prevalence of sarcopenic obesity in adults with end-stage knee osteoarthritis. Osteoarthritis Cartilage. 2019;27(12):1735-45.

103. Vieira FC, de Melo Marinho P, Brandão DC, Barbosae e Silva O. Respiratory muscle strength, the six-minute walk test and quality of life in Chagas cardiomyopathy. Physiother Res Int. 2014;19(1):8-15. 
104. Shen L, Ramires F, Martinez F, Bodanese LC, Echeverría LE, Gómez EA, et al. Contemporary Characteristics and Outcomes in Chagasic Heart Failure Compared With Other Nonischemic and Ischemic Cardiomyopathy. Circ Heart Fail. 2017;10(11):e004361. 


\title{
Discriminating sarcopenia in overweight/obese male patients with heart failure: the influence of body mass index
}

\author{
G.W.P.D. Fonseca ${ }^{1}$, Marcelo Rodrigues dos Santos ${ }^{1}$, Francis Ribeiro de Souza ${ }^{1}$, Liliam Takayama ${ }^{2}$, Rosa Maria \\ Rodrigues Pereira ${ }^{2}$, Carlos Eduardo Negrão ${ }^{1,3}$ and Maria-Janieire de Nazaré Nunes Alves $^{1 *}$ \\ ${ }^{1}$ Heart Institute (InCor), University of São Paulo Medical School, Av. Dr. Enéas de Carvalho Aguiar, 44-Cerqueira César, CEP, 05403-90(4), São Paulo, Brazil; ${ }^{2}$ Rheumatology \\ Division, Bone Metabolism Laboratory, University of São Paulo Medical School, São Paulo, Brazil; ${ }^{3}$ School of Physical Education and Sport, University of São Paulo, São Paulo, \\ Brazil
}

\section{Abstract}

Aims The definition of sarcopenia based on appendicular lean mass/height ${ }^{(2)}$ (ALM/height ${ }^{(2)}$ ) is often used, although it can underestimate the prevalence of sarcopenia in overweight/obese patients with heart failure. Therefore, new methods have been proposed to overcome this limitation. We aimed to evaluate the prevalence of sarcopenia by three methods and compare body composition in this population.

Methods and results We enrolled 168 male patients with heart failure (left ventricular ejection fraction <40\%). Sixty-six patients (39.3\%) were identified with sarcopenia by at least one method. The lower 20th percentile defined as the cut-off point for sarcopenia was $7.03 \mathrm{~kg} / \mathrm{m}^{2},-2.32$ and 0.76 for Baumgartner's (20.8\%), Newman's (21.4\%), and Studenski's methods (21.4\%), respectively. Patients with body mass index (BMI) $<25 \mathrm{~kg} / \mathrm{m}^{2}$ were more likely to be identified by Baumgartner's than Studenski's method $(P<0.001)$. However, in patients with BMI $\geq 25 \mathrm{~kg} / \mathrm{m}^{2}$, Studenski's and Newman's methods were more likely to detect sarcopenia than Baumgartner's method (both $P<0.005$ ). Patients were further divided into three subgroups: (i) patients classified in all indexes $(n=8)$, (ii) patients classified in Baumgartner's (sarcopenic; $n=27$ ), and (iii) patients classified in both Newman's and Studenski's methods (sarcopenic obesity; $n=31$ ). Comparing body composition among groups, all sarcopenic groups presented lower total lean mass compared with non-sarcopenic patients, whereas sarcopenic obese patients had higher total lean mass than lean sarcopenic patients.

Conclusions Our results demonstrate that the prevalence of sarcopenia in overweight/obese patients is similar to lean sarcopenic patients when other methods are considered. In patients with higher BMI, Studenski's method seems to be more feasible to detect sarcopenia.

Keywords Heart failure; Sarcopenia; Sarcopenic obesity; Body composition

Received: 29 July 2019; Accepted: 29 September 2019

*Correspondence to: Maria-Janieire de Nazaré Nunes Alves, Heart Institute (InCor), University of São Paulo Medical School, Av. Dr. Enéas de Carvalho Aguiar, 44-Cerqueira César, CEP 05403-90 (4) São Paulo, Brazil. Tel: +55 11 2661-5099; Fax: +55 11 2661-5043. Email: janieire.alves@incor.usp.br

\section{Introduction}

Sarcopenia has been defined as the age-related decline in skeletal muscle mass, strength, and physical performance. ${ }^{1}$ Sarcopenia affects about $20 \%$ of patients suffering from heart failure (HF) and is a strong predictor of reduced exercise capacity and mortality in these patients. ${ }^{2,3}$
Despite the large-scale use of the term 'sarcopenia', precise criteria to predict clinically relevant reduction in muscle mass and function have not been established yet. In fact, the classic cut-off point of $7.26 \mathrm{~kg} / \mathrm{m}^{2}$ for men, calculated as appendicular lean mass (ALM) in kilograms divided by height in metres squared ( $\mathrm{ALM} /$ height $^{2}$ ), is still frequently used to classify patients with sarcopenia. ${ }^{4}$ Nonetheless, this 
definition does not include the amount of fat mass to predict sarcopenia in overweight and obese patients. ${ }^{5,6}$

Moreover, the combination of reduced muscle mass and fat accumulation, a phenomenon known as sarcopenic obesity (SO), leads to a higher-risk group with both disorders, ${ }^{6,7}$ and men may have a higher prevalence of SO than women. ${ }^{8}$ Paradoxically, although obesity increases the risk of HF, obese patients with HF with reduced ejection fraction (HFrEF) may have lower mortality rate than patients with healthy standard body mass index (BMI; 18.5-24.99 kg/m²). ${ }^{9}$ However, the protective effect of higher $\mathrm{BMI}$ is abolished in the presence of reduced lean mass and increased fat mass. ${ }^{10,11}$

To overcome the limitation of ALM/height ${ }^{2}$ (Baumgartner's method) to define sarcopenia in overweight/obese patients, other definitions have been suggested. For instance, the residuals of a linear regression derived from adjusting lean mass for height and fat mass (Newman's method) seem to be more accurate in patients with high BMI, mainly composed of fat mass., ${ }^{6,12}$ In addition, adjusting ALM by BMI (Studenski's method) has recently been proposed as an alternative to detect SO. ${ }^{13,14}$ Even though both methods may be similar to identify patients with SO, the latter may have a better applicability.

Therefore, the aim of this study was to compare the prevalence of sarcopenia and SO using Baumgartner's, Newman's, and Studenski's methods and to compare body composition features in patients with HFrEF classified by these methods.

\section{Methods}

\section{Study population}

We prospectively included 168 ambulatory male patients with stable HFrEF. This is an unicentric, cross-sectional study conducted between (May) 2016 and November 2018. Patients were eligible to participate if they had (i) age from 18 to 65 years old; (ii) at least 1 year of diagnosed HF; (iii) left ventricular ejection fraction $(<) 40 \%$ measured by echocardiography; (iv) non-ischaemic and ischaemic aetiologies; (v) New York Heart Association Functional Classes I to IV; and (vi) compensated HF with optimal medication for at least 1 month prior to the study.

Patients with autonomic diabetic neuropathy, chronic renal failure in haemodialysis, heart transplantation, cardiac implantable devices, muscular dystrophy (i.e. Duchenne muscular dystrophy), any hormonal treatment, history of cancer, ongoing infection, and myocardial infarction with percutaneous coronary intervention or revascularization 6 months prior to the study entry were not included.

Written informed consent was obtained from all patients before any study-related procedure if they agreed to participate. The study was approved by the local ethics committee
(No. 0892/07) and fulfilled all principles of the Declaration of Helsinki. This protocol is registered at ClinicalTrials.gov under the Unique Identifier Number NCT03463226.

\section{Maximal cardiopulmonary exercise test}

Symptom-limited cardiopulmonary exercise test (Vmax Encore 29 System; VIASYS Healthcare Inc., Palm Springs, CA, USA) was performed on a cycle ergometer by all patients (Ergometer 800S; Sensor Medics, Yorba Linda, CA, USA), using a progressive protocol with workload increments of 5 or $10 \mathrm{~W} / \mathrm{min}$. Ventilation (VE), oxygen consumption $\left(\mathrm{VO}_{2}\right)$, and carbon dioxide output $\left(\mathrm{VCO}_{2}\right)$ were acquired on a breath-by-breath basis and expressed as $30 \mathrm{~s}$ averages. The patients were initially monitored for $2 \mathrm{~min}$ at rest when seated on the ergometer, and after that, they were instructed to pedal at a pace of 60-70 rpm, and the completion of the test occurred when, despite verbal encouragement, the patient reached maximal volitional fatigue. Respiratory exchange ratio higher than 1.10 was reached by $82 \%$ of the patients. Heart rate was monitored continuously at rest, during the test and recovery phase, using a 12-lead digital electrocardiogram (Cardio Soft 6.51 ECG/CAM-14, GE Medical Systems Information Technologies, Wisconsin, WI, USA).

\section{Body composition and muscle strength}

Body composition measurements were performed using dual-energy X-ray absorptiometry (DXA; Lunar iDXA; GE Medical Systems Lunar, Madison, WI, USA). Whole-body DXA scans were used to measure total and regional lean and fat mass. The height of each patient was measured to the nearest $0.1 \mathrm{~cm}$ with a wall-mounted stadiometer. DXA measurements were performed by the same experienced technician.

First, sarcopenia was calculated as the sum of ALM of both arms and legs in kilograms divided by height in metres squared (ALM/height ${ }^{2}$ ), following the method proposed by Baumgartner. ${ }^{4}$ Second, a linear regression was performed using lean mass as dependent variable and height and total fat mass as independent variables, using the residuals to define sarcopenia as proposed by Newman. ${ }^{12}$ In our sample, the equation was $\operatorname{ALM}(\mathrm{kg})=-32.94+31.00 \times$ height $(\mathrm{m})+0.18 \times$ total fat mass $(\mathrm{kg})$. Third, ALM was divided by BMI as proposed by Studenski. ${ }^{13}$ The cut-off values for sarcopenia in this sample were defined as the lower 20th percentile of the distribution of each method.

After adjusting handle position, muscle strength was assessed by handgrip dynamometer (Model J00105; Jamar Hydraulic Hand Dynamometer, Sammons preston rolyan, Bolingbrook, Illionis, USA) using the dominant hand in a supinated position with elbow flexed at $90^{\circ}$. There was $1 \mathrm{~min}$ 
rest interval between efforts, and the maximum value of three attempts was used. ${ }^{15}$ The handgrip cut-off point for sarcopenia was defined according to the BMI categories as proposed by the European Working Group on Sarcopenia in Older People. ${ }^{16}$ We defined sarcopenia as having a cut-off value below the 20th percentile and reduced handgrip strength according to $\mathrm{BMI}$ ranges.

\section{Laboratory measurements}

Blood samples were drawn in the morning after $12 \mathrm{~h}$ overnight fasting. The laboratory tests included B-type natriuretic peptide $(\mathrm{pg} / \mathrm{mL}$ ) plasma level, serum sodium $(\mathrm{mEq} / \mathrm{L})$, serum potassium $(\mathrm{mEq} / \mathrm{L})$, creatinine $(\mathrm{mg} / \mathrm{dL})$, haemoglobin level $(\mathrm{g} / \mathrm{dL})$, high-sensitivity C-reactive protein (hs-CRP; $\mathrm{mg} / \mathrm{L}$ ), and fasting glucose $(\mathrm{mg} / \mathrm{dL})$.

\section{Statistical analysis}

Data are presented as mean \pm standard deviation, median with lower and upper quartiles (95\% confidence interval), and categorical variables as frequencies and percentages. One sample Kolmogorov-Smirnov test was used to assess the normal distribution of all variables. Independent Student's $t$-test and Mann-Whitney $U$ test were used to compare parametric and nonparametric variables, respectively. One-way analysis of variance, Kruskal-Wallis, and $\chi^{2}$ tests were used as appropriate. Data were analysed using the Statistical Package for the Social Sciences Version 23 for Windows (SPSS Inc., Chicago, IL, USA). A P-value lower than 0.05 was considered statistically significant for all analyses.

\section{Results}

We enrolled 168 male patients with stable HFrEF (Table 1). Sixty-six patients $(39.3 \%)$ were identified with sarcopenia by at least one method, using the 20th percentile defined as the cut-off point of $7.03 \mathrm{~kg} / \mathrm{m}^{2},-2.32$ and 0.76 for Baumgartner's, Newman's, and Studenski's methods, respectively. Of those 66 sarcopenic patients, there were eight patients exclusively classified by Baumgartner's method, six patients by Newman's method, and 19 patients by Studenski's method (Figure 1). Twenty-five patients were classified in two of the three methods, which included three patients in Baumgartner's and Studenski's methods, 16 patients in Baumgartner's and Newman's methods, and six patients in Newman's and Studenski's methods (Figure 1). The remaining eight patients were classified in all methods (Figure 1). When using only one of the methods to detect sarcopenia, there were 35 patients in Baumgartner's method (20.8\%), 36

Table 1 Baseline characteristics of the patients with and without sarcopenia

\begin{tabular}{|c|c|c|c|c|}
\hline Variable & All patients $(n=168)$ & Without sarcopenia $(n=102)$ & With sarcopenia ${ }^{a}(n=66)$ & $P$-value \\
\hline Age (years) & $58(51-62)$ & $56(50-61)$ & $60(55-63)$ & $<0.001$ \\
\hline Weight (kg) & $71.5 \pm 13.1$ & $74.5 \pm 10.5$ & $66.8 \pm 14.9$ & $<0.001$ \\
\hline Height (m) & $1.67 \pm 0.07$ & $1.68 \pm 0.06$ & $1.64 \pm 0.07$ & $<0.001$ \\
\hline BMI $\left(\mathrm{kg} / \mathrm{m}^{2}\right)$ & $25.7 \pm 4.2$ & $26.2 \pm 3.0$ & $24.9 \pm 5.3$ & 0.04 \\
\hline Aetiology (ischaemic/non-ischaemic) & $48 / 120$ & $26 / 76$ & $22 / 44$ & 0.30 \\
\hline Aetiology (Chagas/no Chagas) & $40 / 128$ & $26 / 76$ & $14 / 52$ & 0.58 \\
\hline NYHA class (I/II/III/IV) & $54 / 57 / 46 / 11$ & $(39 / 31 / 24 / 8)$ & $(15 / 26 / 22 / 3)$ & 0.20 \\
\hline LVEF (\%) & $27(22-33)$ & $27(22-33)$ & $25(21-34)$ & 0.82 \\
\hline $\mathrm{BNP}(\mathrm{pg} / \mathrm{mL})$ & $438(147-1050)$ & $363(123-993)$ & $461(154-1336)$ & 0.54 \\
\hline hs-CRP (mg/L) & $2.8(1.0-7.1)$ & $2.4(0.9-5.9)$ & $3.3(1.2-12.7)$ & 0.049 \\
\hline Creatinine (mg/dL) & $1.1(1.0-1.5)$ & $1.2(1.0-1.5)$ & $1.1(1.0-1.4)$ & 0.12 \\
\hline Sodium (mEq/L) & $140(138-141)$ & $140(138-141)$ & $139(138-141)$ & 0.75 \\
\hline Potassium (mEq/L) & $4.5(4.3-4.8)$ & $4.5(4.3-4.7)$ & $4.6(4.3-4.9)$ & 0.16 \\
\hline Haemoglobin (g/dL) & $13.9 \pm 1.8$ & $14.0 \pm 1.8$ & $14.0 \pm 1.5$ & 0.84 \\
\hline Fasting glucose $(\mathrm{mg} / \mathrm{dL})$ & $104(95-115)$ & $104(95-114)$ & $104(95-119)$ & 0.87 \\
\hline \multicolumn{5}{|l|}{ Medication } \\
\hline Beta-blocker, $n$ (\%) & $164(98)$ & $99(97)$ & $65(98)$ & 1.00 \\
\hline ACE-I/ARB, $n(\%)$ & $153(91)$ & $95(93)$ & $58(88)$ & 0.28 \\
\hline Thiazide/loop diuretics, $n$ (\%) & $132(79)$ & $80(78)$ & $52(79)$ & 1.00 \\
\hline Aspirin, $n(\%)$ & $66(39)$ & $34(33)$ & $32(48)$ & 0.06 \\
\hline Statins, $n(\%)$ & $91(54)$ & $51(50)$ & $40(61)$ & 0.21 \\
\hline MRA, $n(\%)$ & $118(70)$ & $71(70)$ & $47(71)$ & 0.86 \\
\hline Warfarin, $n(\%)$ & $44(26)$ & $32(31)$ & $12(18)$ & 0.07 \\
\hline Vasodilators, $n(\%)$ & $49(29)$ & $32(31)$ & $17(26)$ & 0.49 \\
\hline Metformin, $n(\%)$ & $22(13)$ & $11(11)$ & $11(17)$ & 0.35 \\
\hline
\end{tabular}

ACE-I, angiotensin-converting enzyme inhibitor; ARB, angiotensin receptor blocker; BMI, body mass index; BNP, B-type natriuretic peptide; hs-CRP, high-sensitivity C-reactive protein; LVEF, left ventricular ejection fraction; MRA, mineralocorticoid receptor antagonist; NYHA, New York Heart Association.

Data are presented as mean \pm SD, median (with lower and upper quartiles), or frequencies and percentages.

apatients classified by all methods. 
Figure 1 Prevalence of sarcopenia according to Baumgartner's method (circle 1), Newman's method (circle 2), and Studenski's method (circle 3). Patients were further divided into three subgroups based on body composition. Number in black intersected by all circles refers to patients included in all indexes $(n=8)$. Numbers in green represent patients in the sarcopenic group $(n=27)$. Numbers in red represent patients in the sarcopenic obesity group $(n=31)$.

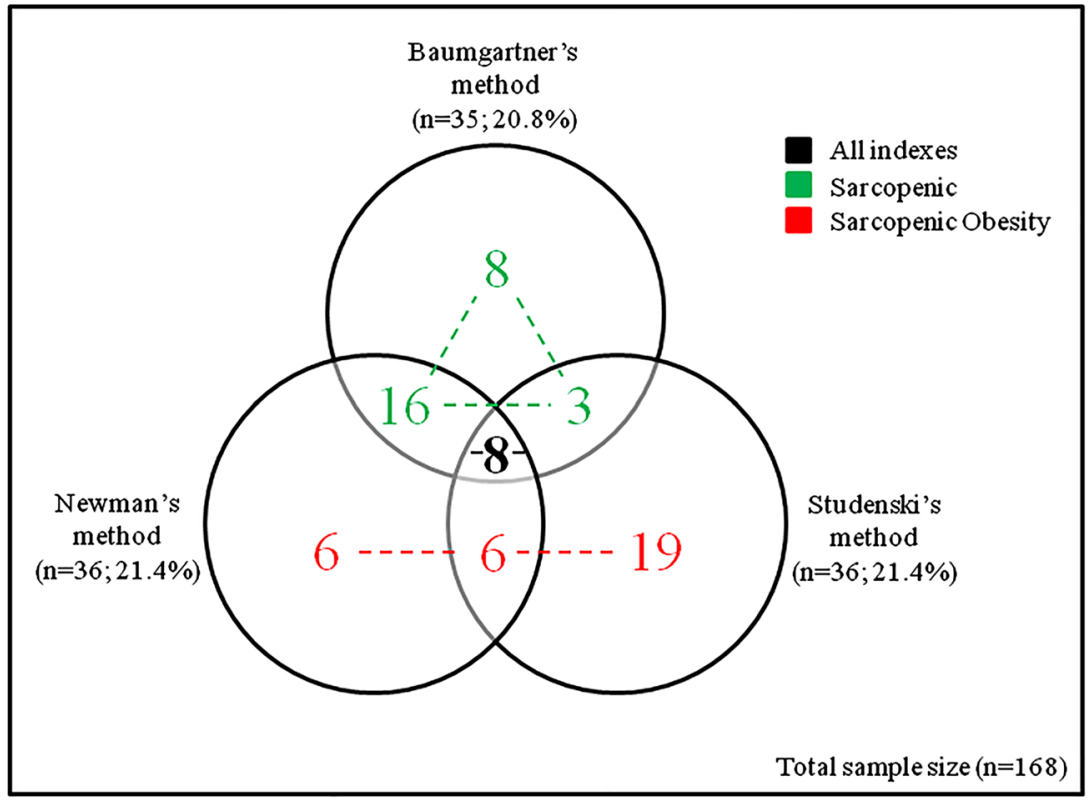

patients in Newman's method (21.4\%), and 36 patients in Studenski's method (21.4\%) (Figure 1).

The distribution of individuals according to the BMI categories is presented in Figure 2. Patients with BMI $<25 \mathrm{~kg} / \mathrm{m}^{2}$ were more likely to be identified as sarcopenic by Baumgartner's method ( $n=31 ; 18.5 \%$ ) when compared with Studenski's method ( $n=12 ; 7.1 \% ; P<0.001$ ), and there was a trend towards higher number of cases in Baumgartner's method compared with Newman's method ( $n=20 ; 11.9 \%$;

Figure 2 Prevalence of sarcopenia by method in patients with body mass index (BMI) $<25$ and $\geq 25 \mathrm{~kg} / \mathrm{m}^{2}$. ${ }^{*} P=0.053$ vs. Newman's method. ${ }^{\dagger} P<$ 0.001 vs. Studenski's method. ${ }^{\ddagger} P<0.005$ vs. both Newman's and Studenski's methods.

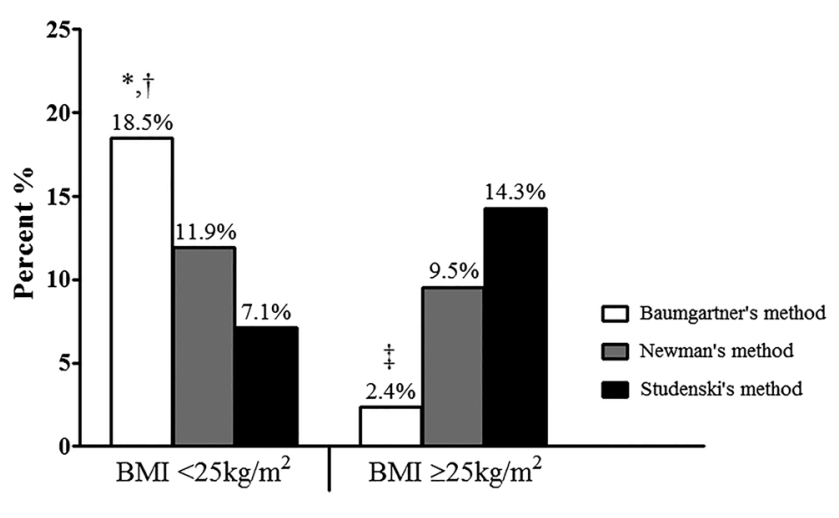

$P=0.053)$. Patients with $\mathrm{BMI} \geq 25 \mathrm{~kg} / \mathrm{m}^{2}$ were more likely to be identified as sarcopenic by Studenski's ( $n=24 ; 14.3 \%$ ) and Newman's methods ( $n=16 ; 9.5 \%$ ) when compared with Baumgartner's method ( $n=4 ; 2.4 \%$; both $P<0.005)$. There was no difference between Studenski's and Newman's methods in both categories of BMI $(P=0.11 ; P=0.16)$.

Regardless of method used for definition, patients with sarcopenia were older and had higher hs-CRP when compared with non-sarcopenic patients. In addition, weight, $\mathrm{BMI}$, and height were lower in sarcopenic patients compared with non-sarcopenic. There was no statistical difference in medication between groups (Table 1).

We further divided the 66 sarcopenic patients into three subgroups (Table 2 and Figure 1) according to body composition features, which consisted of patients classified in all indexes $(n=8)$, patients classified in Baumgartner's method (sarcopenic; $n=27$ ) and patients classified in Newman's plus Studenski's method (sarcopenic obesity; $n=31$ ).

Table 2 shows clinical characteristics of patients included in the subgroups of sarcopenia. In general, we observed that lean sarcopenic patients presented lower exercise tolerance (reduced absolute peak $\mathrm{VO}_{2}$ but not relative peak $\mathrm{VO}_{2}$ ) and higher B-type natriuretic peptide and hs-CRP when compared with non-sarcopenic and sarcopenic obese patients. On the other hand, lean sarcopenic patients showed normal glucose levels when compared with both non-sarcopenic and sarcopenic obese patients. Left ventricular ejection fraction was not different among groups (Table 2). 
Table 2 Demographic and clinical characteristics in subgroups of sarcopenia

\begin{tabular}{|c|c|c|c|c|}
\hline Variable & $\begin{array}{l}\text { Without sarcopenia } \\
\qquad(n=102)\end{array}$ & $\begin{array}{l}\text { All indexes } \\
(n=8)\end{array}$ & $\begin{array}{l}\text { Sarcopenic } \\
(n=27)\end{array}$ & $\begin{array}{c}\text { Sarcopenic obesity } \\
(n=31)\end{array}$ \\
\hline Age (years) & $56(50-61)$ & $63(56-68)^{*}$ & $61(57-63)^{*}$ & $59(55-63)^{*}$ \\
\hline Weight $(\mathrm{kg})$ & $74.5 \pm 10.5$ & $56.3 \pm 9.6^{*}, t$ & $58.4 \pm 7.8^{*}, t$ & $76.8 \pm 14.5$ \\
\hline Height $(\mathrm{m})$ & $1.68 \pm 0.06$ & $1.59 \pm 0.05^{* \neq}$ & $1.67 \pm 0.06^{\dagger}$ & $1.62 \pm 0.07^{*}$ \\
\hline BMI $\left(\mathrm{kg} / \mathrm{m}^{2}\right)$ & $26.2 \pm 3.0$ & $22.3 \pm 3.1^{*, \dagger}$ & $20.9 \pm 2.3^{*, \dagger}$ & $29.1 \pm 4.4^{*}$ \\
\hline Aetiology (ischaemic/non-ischaemic) & $26 / 76$ & $2 / 6$ & $6 / 21$ & $14 / 17$ \\
\hline Aetiology (Chagas/no Chagas) & $26 / 76$ & $1 / 7$ & $8 / 19$ & $5 / 26$ \\
\hline NYHA class (I/II/III/IV) & $(39 / 31 / 24 / 8)$ & $1 / 3 / 4 / 0$ & $8 / 11 / 7 / 1$ & $6 / 12 / 11 / 2$ \\
\hline $\operatorname{LVEF}(\%)$ & $27(22-33)$ & (25) $(21-37)$ & $25(20-31)$ & $28(21-33)$ \\
\hline $\mathrm{BNP}(\mathrm{pg} / \mathrm{mL})$ & $363(123-993)$ & $986(299-2073)^{\dagger}$ & $647(207-1635)^{\dagger}$ & 201 (99-784) \\
\hline hs-CRP (mg/L) & $2.4(0.9-5.9)$ & $9.2(3.1-29.2)^{\star}$ & $3.0(1.2-12.4)$ & $3.8(0.9-9.1)$ \\
\hline Creatinine $(\mathrm{mg} / \mathrm{dL})$ & $1.2(1.0-1.5)$ & $1.4(1.1-2.0)^{t, \ddagger}$ & $1.1(0.9-1.3)$ & $1.1(1.0-1.4)$ \\
\hline Sodium (mEq/L) & $140(138-141)$ & $140(138-142)$ & $139(137-141)$ & $140(138-142)$ \\
\hline Potassium (mEq/L) & $4.5(4.3-4.7)$ & $4.5(4.2-4.9)$ & $4.6(4.2-4.7)$ & $4.7(4.4-4.9)$ \\
\hline Haemoglobin $(\mathrm{g} / \mathrm{dL})$ & $14.0 \pm 1.8$ & $13.2 \pm 1.2$ & $13.8 \pm 1.5$ & $14.3 \pm 1.5$ \\
\hline Fasting glucose (mg/dL) & $104(95-114)$ & $97(91-110)^{*+\dagger}$ & $98(88-108)^{*, \dagger}$ & $114(102-130)^{*}$ \\
\hline \multicolumn{5}{|l|}{ Functional capacity and strength } \\
\hline Peak $\mathrm{VO}_{2}(\mathrm{~L} / \mathrm{min})$ & $1.41(1.01-1.81)$ & $0.96(0.90-1.06)^{*, \dagger}$ & $0.98(0.85-1.31)^{*, \dagger}$ & $1.25(1.09-1.73)$ \\
\hline Peak $\mathrm{VO}_{2}(\mathrm{~mL} / \mathrm{min} / \mathrm{kg})$ & $19.3(13.9-24.7)$ & $16.0(14.7-17.5)$ & $17.0(14.3-23.9)$ & $16.6(14.1-22.0)$ \\
\hline VE $/ \mathrm{VCO}_{2}$ slope & $34(29-39)$ & $33(26-36)$ & $36(32-40)$ & $36(31-44)$ \\
\hline Peak workload (W) & $110(70-150)$ & $70(38-88) *$ & $70(60-90)^{*}$ & $90(55-115)^{*}$ \\
\hline Time $(s)$ & $556(475-664)$ & $590(469-783)$ & $510(379-569)^{* \dagger}$ & $572(509-663)$ \\
\hline Handgrip strength $(\mathrm{kg})$ & $34.6 \pm 7.6$ & $25.4 \pm 5.5^{*},(\dagger)$ & $29.6 \pm 6.8^{*}$ & $33.2 \pm 6.1$ \\
\hline
\end{tabular}

BMI, body mass index; BNP, B-type natriuretic peptide; hs-CRP, high-sensitivity C-reactive protein; LVEF, left ventricular ejection fraction; NYHA, New York Heart Association; $\mathrm{VE} / \mathrm{VCO}_{2}$, ventilatory equivalent for carbon dioxide; $\mathrm{VO}_{2}$, oxygen consumption.

Data are presented as mean \pm SD or median (with lower and upper quartiles). Symbols in brackets denote a trend with $P<0.10$.

${ }^{*} P<0.05$ (vs. without sarcopenia).

${ }^{\dagger} P<0.05$ (vs. sarcopenic obesity).

${ }^{\ddagger} P<0.05$ (vs. sarcopenic).

\section{Body composition}

Patients included in the sarcopenic group had a lower total fat mass, fat percentage, fat mass in arms, legs, and trunk when compared with non-sarcopenic patients and sarcopenic obese patients. In addition, sarcopenic obese patients presented higher total fat mass, fat percentage, fat mass in arms, legs, and trunk than those without sarcopenia and classified in all indexes (Table 3).
Despite the method used to define sarcopenia, all sarcopenic groups presented lower total lean mass and reduced lean mass in arms, legs, and trunk compared with non-sarcopenic patients, whereas sarcopenic obese patients had higher total lean mass and lean mass in arms and legs than patients classified in all indexes and in the sarcopenic group. In addition, lean percentage and lean/fat mass ratio were lower in sarcopenic obese patients when compared with their sarcopenic counterparts and non-sarcopenic

Table 3 Body composition in subgroups of sarcopenia

\begin{tabular}{|c|c|c|c|c|}
\hline Variable & Without sarcopenia $(n=102)$ & All indexes $(n=8)$ & Sarcopenic $(n=27)$ & Sarcopenic obesity $(n=31)$ \\
\hline \multicolumn{5}{|l|}{ Fat mass (kg) } \\
\hline Arms & $1.9 \pm 0.6$ & $1.7 \pm 0.4^{\dagger}$ & $1.4 \pm 0.5^{*, \dagger}$ & $2.7 \pm 1.1^{*}$ \\
\hline Legs & $5.5 \pm 2.3$ & $4.3 \pm 1.0^{(*), \dagger}$ & $4.2 \pm 1.2^{*, \dagger}$ & $6.6 \pm 1.9^{*}$ \\
\hline Trunk & $10.6 \pm 4.9$ & $8.5 \pm 2.8^{\dagger}$ & $6.5 \pm 4.0^{*, \dagger}$ & $15.9 \pm 5.4^{*}$ \\
\hline Total & $18.4 \pm 7.0$ & $15.2 \pm 4.1^{\dagger}$ & $12.8 \pm 5.6^{* \dagger}$ & $26.2 \pm 8.1 *$ \\
\hline Total fat (\%) & $25 \pm 7$ & $28 \pm 5^{\dagger, t}$ & $22 \pm 7^{*, \dagger}$ & $35 \pm 5^{*}$ \\
\hline \multicolumn{5}{|l|}{ Lean mass (kg) } \\
\hline Arms & $6.5 \pm 0.9$ & $4.2 \pm 0.6^{* \prime \dagger}$ & $5.0 \pm 0.6^{*+\dagger}$ & $5.9 \pm 0.8^{*}$ \\
\hline Legs & $17.4 \pm 2.7$ & $11.3 \pm 1.4^{* \dagger}$ & $13.2 \pm 1.4^{* \dagger}$ & $15.4 \pm 2.5^{*}$ \\
\hline Trunk & $26.0 \pm 4.3$ & $20.3 \pm 4.6^{*}$ & $21.7 \pm 2.2^{*}$ & $23.4 \pm 4.3^{*}$ \\
\hline Total & $52.4 \pm 5.8$ & $38.0 \pm 5.9^{*, \dagger}$ & $42.6 \pm 4.1^{*},(\dagger)$ & $46.3 \pm 4.0 *$ \\
\hline Total lean (\%) & $71 \pm 6$ & $68 \pm 4^{(*), t, \ddagger}$ & $73 \pm 6^{\dagger}$ & $63 \pm 5^{*}$ \\
\hline Lean mass/fat mass & $3.3 \pm 1.6$ & $2.7 \pm 0.8^{\dagger, \ddagger}$ & $3.9 \pm 1.5^{*, \dagger}$ & $2.0 \pm 0.5^{*}$ \\
\hline
\end{tabular}

Data are presented as mean \pm SD or median (with lower and upper quartiles). Symbols in brackets denote a trend with $P<0.10$.

${ }^{*} P<0.05$ (vs. without sarcopenia).

${ }^{\dagger} P<0.05$ (vs. sarcopenic obesity).

${ }^{\ddagger} P<0.05$ (vs. sarcopenic). 
patients. Patients classified in all indexes showed lower lean percentage and lean/fat mass ratio than lean sarcopenic patients (Table 3).

\section{Discussion}

The main findings of this study are that the criteria based on ALM adjusted by height plus fat mass (Newman's method) or adjusted by BMI (Studenski's method) can be used to determine sarcopenia in overweight/obese patients with HFrEF, but the latter seems to be more appropriate to apply in the clinical setting. The prevalence of sarcopenia alone and $\mathrm{SO}$ is similar in patients with HFrEF when using methods that consider fat mass and body size in its equation. Moreover, as expected, patients with HFrEF and SO present higher lean mass than lean sarcopenic patients, although the amount of lean mass is still lower than nonsarcopenic patients.

Sarcopenia has become a major public health issue as the population ages. It is well recognized as an independent marker of worse prognosis in patients with $\mathrm{HF}^{2}$ and has been related to reduced functional capacity and sympathovagal imbalance. ${ }^{17,18}$ The last revised European consensus about sarcopenia states that the cut-off point for sarcopenia must be lower than $7.0 \mathrm{~kg} / \mathrm{m}^{2}$ for men using Baumgartner's method. ${ }^{1}$ In our study, using the lower 20th percentile of Baumgartner's method to define sarcopenia, we found a similar cut-off of $7.03 \mathrm{~kg} / \mathrm{m}^{2}$. In addition, the cut-off of Newman's method and Studenski's method was -2.32 and 0.76 , respectively. They were also very close to what have been proposed by others. ${ }^{12,13}$

In a sample of 200 patients with HF, Fülster and colleagues found that 39 patients (19.5\%) were classified as sarcopenic, and about 37 of these patients (94.9\%) were men. In our study, the prevalence of sarcopenia was $20.8 \%$ for Baumgartner's method and $21.4 \%$ for Newman's and Studenski's methods, showing a very similar prevalence among the three methods and also close to the percentage described in some studies ${ }^{19,20}$ but a little higher than in other populations. $^{14}$

In addition, obesity, defined as BMI $\geq 30 \mathrm{~kg} / \mathrm{m}^{2}$, has reached epidemic proportions and is known to increase the risk of developing HF. ${ }^{21}$ However, once HF is established, obese patients may have an improved outcome because of the obesity paradox. ${ }^{10}$ Although $\mathrm{BMI}$ is high, this protective effect of overweight/obesity in these patients may be abolished in the presence of decreased lean mass and increased fat mass. ${ }^{10,11}$ Moreover, increases in BMI cannot detect the regional body fat mass distribution, especially visceral fat that has been associated with mortality in these patients. ${ }^{22}$ Furthermore, the protective effect of higher BMI should be considered with markers of functional capacity, once the obesity paradox has been described to be more significant in patients with lower cardiorespiratory fitness. ${ }^{23}$

In spite of the limitations involved with BMI, we can still use it to determine the relation between lean and fat mass, once ALM is measured by a gold standard method to determine body composition (DXA). ${ }^{24}$ In our study, we found that using Studenski's method to determine SO was alike Newman's method, which uses a linear regression and adds fat mass to the index proposed by Baumgartner to diagnose sarcopenia. Furthermore, considering the similarities between Newman's and Studenski's methods in predicting SO in patients with HFrEF, BMI can be an easy and applicable tool to adjust ALM in patients with higher body weight, although neither of these methods is superior to the other.

The combination of sarcopenia and obesity has been described as a higher-risk group that can potentiate the adverse effects of these conditions alone. ${ }^{6,7}$ Low-grade inflammation seems to be a common pathway in the progression of these disorders and leads to alterations in body composition. ${ }^{25,26}$ Regardless of the definition of sarcopenia, we found that sarcopenic patients presented increased hs-CRP. Although we only measured one inflammatory marker, previous studies have shown that hs-CRP enables detection of even mild alterations in inflammation. ${ }^{27}$

Moreover, muscle mass seems to be increased in patients with SO because of a higher body weight they have to carry, and it leads to a better maintenance of lean mass. In our study, although patients with so presented higher lean mass compared with lean sarcopenic patients, percentage lean mass and lean/fat mass ratio, used to correct muscle mass in relation to body weight and fat, were reduced in these patients. On the other hand, even though peak workload was lower in all sarcopenic patients, strength and peak $\mathrm{VO}_{2}$ were similar between patients with SO and non-sarcopenic. These findings imply that some components of functional capacity and body composition have already been compromised in these patients, whilst others are still preserved. Physical activity has extensively shown to improve health status in patients with HF, and it may play a role in reducing the risk of sarcopenia and SO in the general population. ${ }^{28,29}$

\section{Limitations}

Despite the novel findings presented in this study, some limitations must be addressed. First, we only included male patients with HFrEF, so we are not able to extrapolate these results to women and patients with HF with preserved ejection fraction. Second, the cut-off value of 20th percentile was arbitrary, although it has been used in larger trials than ours. Finally, there was no follow-up to evaluate the impact of these alterations on prognosis in these patients, and the cross-sectional analysis of this study does not allow conclusions about cause-and-effect relationships. 


\section{Conclusions}

In summary, our results demonstrate that the prevalence of sarcopenia in overweight/obese patients is similar to lean sarcopenic patients when appropriate definitions are used to determine sarcopenia in male patients with HFrEF. In patients with higher BMI, Studenski's method seems to be more feasible to detect sarcopenia in these patients.

\section{Funding}

This study was funded by Fundação de Amparo à Pesquisa do Estado de São Paulo (FAPESP; 2015/22814-5). G.W.P.d.F. was supported by Conselho Nacional de Desenvolvimento Científico e Tecnológico (CNPq; 148758/2016-9); M.R.d.S. by FAPESP (2016/24306-0); and C.E.N. by FAPESP (2015/228145) and CNPq (303573/2015-5). FAPESP is from São Paulo, Brazil. CNPq is a national institution that supports research in Brazil.

\section{Conflict of interest}

None declared.

\section{References}

1. Fülster S, Tacke M, Sandek A, Ebner N, Tschöpe C, Doehner W, Anker SD, von Haehling S. Muscle wasting in patients with chronic heart failure: results from the studies investigating co-morbidities aggravating heart failure (SICA-HF). Eur Heart $J$ 2013; 34: 512-519.

2. Cruz-Jentoft AJ, Bahat G, Bauer J, Boirie $\mathrm{Y}$, Bruyère $\mathrm{O}$, Cederholm $\mathrm{T}$, Cooper $\mathrm{C}$, Landi F, Rolland Y, Sayer AA, Schneider SM, Sieber CC, Topinkova E, Vandewoude M, Visser M, Zamboni M. Writing Group for the European Working Group on Sarcopenia in Older People 2 (EWGSOP2) atEGfE. Sarcopenia: revised European consensus on definition and diagnosis. Age Ageing 2019; 48: 16-31.

3. Cicoira M, Zanolla L, Franceschini L, Rossi A, Golia G, Zamboni M, Tosoni P, Zardini P. Skeletal muscle mass independently predicts peak oxygen consumption and ventilatory response during exercise in noncachectic patients with chronic heart failure. $J$ Am Coll Cardiol 2001; 37: 2080-2085.

4. Baumgartner RN, Koehler KM, Gallagher D, Romero L, Heymsfield SB, Ross RR, Garry PJ, Lindeman RD. Epidemiology of sarcopenia among the elderly in New Mexico. Am J Epidemiol 1998; 147: 755-763.

5. Delmonico MJ, Harris TB, Lee JS, Visser M, Nevitt M, Kritchevsky SB, Tylavsky FA, Newman AB, Health AiaBCS. Alternative definitions of sarcopenia, lower extremity performance, and functional impairment with aging in older men and women. J Am Geriatr Soc 2007; 55: 769-774.

6. Kim TN, Yang SJ, Yoo HJ, Lim KI, Kang HJ, Song W, Seo JA, Kim SG, Kim NH, Baik SH, Choi DS, Choi KM. Prevalence of sarcopenia and sarcopenic obesity in Korean adults: the Korean sarcopenic obesity study. Int $J$ Obes (Lond) 2009; 33: 885-892

7. Tian S, Xu Y. Association of sarcopenic obesity with the risk of all-cause mortality: a meta-analysis of prospective cohort studies. Geriatr Gerontol Int 2016; 16 : 155-166.

8. Batsis JA, Mackenzie TA, Barre LK, Lopez-Jimenez F, Bartels SJ. Sarcopenia, sarcopenic obesity and mortality in older adults: results from the National Health and Nutrition Examination Survey III. Eur J Clin Nutr 2014; 68: 1001-1007.

9. Carbone S, Lavie CJ, Arena R. Obesity and heart failure: focus on the obesity paradox. Mayo Clin Proc 2017; 92: 266-279.

10. Thomas E, Gupta PP, Fonarow GC, Horwich TB. Bioelectrical impedance analysis of body composition and survival in patients with heart failure. Clin Cardiol 2019; 42: 129-135.

11. De Schutter A, Lavie CJ, Kachur S, Patel DA, Milani RV. Body composition and mortality in a large cohort with preserved ejection fraction: untangling the obesity paradox. Mayo Clin Proc 2014; 89: 1072-1079.

12. Newman AB, Kupelian V, Visser $M$, Simonsick E, Goodpaster B, Nevitt M, Kritchevsky SB, Tylavsky FA, Rubin SM, Harris TB, Investigators HAS. Sarcopenia: alternative definitions and associations with lower extremity function. J Am Geriatr Soc 2003; 51: 1602-1609.

13. Studenski SA, Peters KW, Alley DE, Cawthon PM, McLean RR, Harris TB, Ferrucci L, Guralnik JM, Fragala MS, Kenny AM, Kiel DP, Kritchevsky SB, Shardell MD, Dam TT, Vassileva MT. The FNIH sarcopenia project: rationale, study description, conference recommendations, and final estimates. $J$ Gerontol A Biol Sci Med Sci 2014; 69: 547-558.
14. Tyrovolas S, Koyanagi A, Olaya B, AyusoMateos JL, Miret M, Chatterji S, TobiaszAdamczyk B, Koskinen S, Leonardi M, Haro JM. Factors associated with skeletal muscle mass, sarcopenia, and sarcopenic obesity in older adults: a multi-continent study. $J$ Cachexia Sarcopenia Muscle 2016; 7: 312-321.

15. Roberts HC, Denison HJ, Martin HJ, Patel HP, Syddall H, Cooper C, Sayer AA. A review of the measurement of grip strength in clinical and epidemiological studies: towards a standardised approach. Age Ageing 2011; 40: 423-429.

16. Cruz-Jentoft AJ, Baeyens JP, Bauer JM, Boirie Y, Cederholm T, Landi F, Martin FC, Michel JP, Rolland Y, Schneider SM, Topinková E, Vandewoude M, Zamboni M, People EWGoSiO. Sarcopenia: European consensus on definition and diagnosis: report of the European Working Group on Sarcopenia in Older People. Age Ageing 2010; 39: 412-423.

17. Fonseca GWPD, Santos MRD, Souza FR, Costa MJAD, Haehling SV, Takayama L, Pereira RMR, Negrão CE, Anker SD, Alves MJNN. Sympatho-vagal imbalance is associated with sarcopenia in male patients with heart failure. Arq Bras Cardiol 2019; 112: 739-746.

18. Suzuki T, Palus S, Springer J. Skeletal muscle wasting in chronic heart failure. ESC Heart Fail. 2018; 5: 1099-1107.

19. Yamada M, Nishiguchi S, Fukutani N, Tanigawa T, Yukutake T, Kayama $\mathrm{H}$, Aoyama T, Arai $H$. Prevalence of sarcopenia in community-dwelling Japanese older adults. J Am Med Dir Assoc 2013; 14: 911-915.

20. Fukuda $T$, Bouchi $R$, Takeuchi $T$, Tsujimoto K, Minami I, Yoshimoto T, Ogawa Y. Sarcopenic obesity assessed using dual energy X-ray absorptiometry (DXA) can predict cardiovascular disease in patients with type 2 diabetes: a 
retrospective observational study. Cardiovasc Diabetol 2018; 17: 55.

21. Nicklas BJ, Cesari M, Penninx BW, Kritchevsky SB, Ding J, Newman A, Kitzman DW, Kanaya AM, Pahor M, Harris TB. Abdominal obesity is an independent risk factor for chronic heart failure in older people. $J$ Am Geriatr Soc 2006; 54: 413-420.

22. Streng KW, Voors AA, Hillege HL, Anker SD, Cleland JG, Dickstein K, Filippatos G, Metra M, Ng LL, Ponikowski P, Samani NJ, van Veldhuisen DJ, Zwinderman AH, Zannad F, Damman K, van der Meer P, Lang CC. Waist-tohip ratio and mortality in heart failure. Eur J Heart Fail 2018; 20: 1269-1277.

23. Clark AL, Fonarow GC, Horwich TB. Impact of cardiorespiratory fitness on the obesity paradox in patients with systolic heart failure. Am J Cardiol. 2015; 115: 209-213.

24. Clark BC, Tavoian D, Goodpaster BH, Cawthon PM, Hansen RD, Manini TM. Pitfalls in the measurement of muscle mass: a need for a reference standard. J Cachexia Sarcopenia Muscle 2018; 9: 1269-1271.

25. Park CH, Do JG, Lee YT, Yoon KJ. Sarcopenic obesity associated with highsensitivity C-reactive protein in age and sex comparison: a two-center study in South Korea. BMJ Open 2018; 8: e021232.

26. Marcus RL, Addison O, Dibble LE, Foreman KB, Morrell G, Lastayo P. Intramuscular adipose tissue, sarcopenia, and mobility function in older individuals. J Aging Res 2012; 2012: 629637.
27. Ridker PM. High-sensitivity C-reactive protein: potential adjunct for global risk assessment in the primary prevention of cardiovascular disease. Circulation 2001; 103: 1813-1818.

28. Ryu M, Jo J, Lee Y, Chung YS, Kim $\mathrm{KM}$, Baek WC. Association of physical activity with sarcopenia and sarcopenic obesity in communitydwelling older adults: the Fourth Korea National Health and Nutrition Examination Survey. Age Ageing 2013; 42: 734-740.

29. Saitoh M, Ishida J, Springer J. Physical activity for the prevention and treatment of sarcopenic obesity. $J$ Cachexia Sarcopenia Muscle 2017; 8: 518-519. 


\title{
Sympatho-Vagal Imbalance is Associated with Sarcopenia in Male Patients with Heart Failure
}

\author{
Guilherme Wesley Peixoto da Fonseca, ${ }^{1 \oplus}$ Marcelo Rodrigues dos Santos, ${ }^{1 \bullet}$ Francis Ribeiro de Souza, ${ }^{1 \oplus}$ Marcel Jose \\ A. da Costa, ${ }^{1}$ Stephan von Haehling, ${ }^{20}$ Liliam Takayama, ${ }^{3 \oplus}$ Rosa Maria R. Pereira, ${ }^{3 \circledR}$ Carlos Eduardo Negrão, ${ }^{10}$ \\ Stefan D. Anker, ${ }^{\circledR 0}$ Maria Janieire de Nazaré Nunes Alves ${ }^{10}$ \\ Instituto do Coração (InCor), ${ }^{1}$ São Paulo, SP - Brazil \\ Department of Cardiology and Pneumology - University of Göttingen Medical Centre, ${ }^{2}$ Göttingen - Germany \\ Divisão de Reumatologia - Laboratório de Metabolismo Ósseo da Faculdade de Medicina da Universidade de São Paulo, ${ }^{3}$ São Paulo, SP - Brazil
}

\begin{abstract}
Background: Resting sympathetic hyperactivity and impaired parasympathetic reactivation after exercise have been described in patients with heart failure (HF). However, the association of these autonomic changes in patients with HF and sarcopenia is unknown.
\end{abstract}

Objective: The aim of this study was to evaluate the impact of autonomic modulation on sarcopenia in male patients with HF.

Methods: We enrolled 116 male patients with $\mathrm{HF}$ and left ventricular ejection fraction $<40 \%$. All patients underwent a maximal cardiopulmonary exercise testing. Maximal heart rate was recorded and delta heart rate recovery $(\Delta H R R)$ was assessed at $1^{\text {st }}$ and $2^{\text {nd }}$ minutes after exercise. Muscle sympathetic nerve activity (MSNA) was recorded by microneurography. Dual-energy X-ray absorptiometry was used to measure body composition and sarcopenia was defined by the sum of appendicular lean muscle mass (ALM) divided by height in meters squared and handgrip strength.

Results: Sarcopenia was identified in 33 patients (28\%). Patients with sarcopenia had higher MSNA than those without (47 [41-52] vs. 40 [34-48] bursts/min, $p=0.028)$. Sarcopenic patients showed lower $\Delta H R R$ at $1^{\text {st }}(15$ [10-21] vs. 22 [16-30] beats/min, $p<0.001)$ and $2^{\text {nd }} \min (25[19-39]$ vs. 35 [24-48] beats/min, $p=0.017)$ than non-sarcopenic. There was a positive correlation between ALM and $\Delta$ HRR at $1^{\text {st }}(r=0.26, p=0.008)$ and $2^{\text {nd }} \min (r=0.25, p=0.012)$. We observed a negative correlation between ALM and MSNA $(r=-0.29, p=0.003)$.

Conclusion: Sympatho-vagal imbalance seems to be associated with sarcopenia in male patients with HF. These results highlight the importance of a therapeutic approach in patients with muscle wasting and increased peripheral sympathetic outflow. (Arq Bras Cardiol. 2019; 112(6):739-746)

Keywords: Heart Failure; Sarcopenia; Sympathetic Hyperactivity; Blunted Vagal Reactivation.

\section{Introduction}

Changes in body composition play an important role in the pathogenesis and progression of chronic heart failure (HF). ${ }^{1}$ Sarcopenia, which is characterized by a decrease in skeletal muscle mass and strength, affects $19.5 \%$ of ambulatory patients with $\mathrm{HF}^{2}$ and is associated with several alterations such as impaired endothelial function, reduced 6-minute walking distance, and attenuated peak $\mathrm{VO}_{2} \cdot{ }^{2,3}$ Although sarcopenia has been frequently described in elderly patients as a consequence of the ageing process, it can also be present in younger patients with $\mathrm{HF}^{4}$

Resting sympathoexcitation is a hallmark in chronic $\mathrm{HF}^{5}$ In addition, accumulated evidence shows that this autonomic

Mailing Address: Maria Janieire de Nazaré Nunes Alves •

Avenida Doutor Enéas de Carvalho Aguiar, 44. Postal Code 05403-900, Jardim Paulista, São Paulo, SP - Brazil

E-mail: janieire.ja@gmail.com, guilhermefonseca@usp.br

Manuscript received June 11, 2018, revised manuscript September 14 2018, accepted October 02, 2018

DOI: $10.5935 / a b c .20190061$ dysregulation is highly associated with increased morbidity and mortality. ${ }^{5}$ In normal conditions, sympathetic nervous system exerts anabolic action via $\beta_{2}$-adrenoceptors on skeletal muscle, ${ }^{6}$ but in experimental model of $\mathrm{HF}$, the exacerbated sympathetic nervous activity contributes to downregulation of $\beta_{2}$-adrenoceptors favoring skeletal muscle atrophy and weight loss.

Reduced parasympathetic activity has also been reported in patients with HF. ${ }^{8,9}$ Binkley and colleagues ${ }^{10}$ showed impaired parasympathetic activity in patients with HF evaluated by heart rate variability. Moreover, heart rate recovery (HRR), an important cardiac deceleration mechanism after maximum effort, can also be used to assess parasympathetic activity immediately after maximal exercise testing. ${ }^{11}$ Furthermore, HRR is an easy, low-cost, and clinical assessment of vagal reactivation, and provides additional prognostic information. ${ }^{12-14}$

Muscle sympathetic nerve activity (MSNA) and HRR, as measures of sympathetic and parasympathetic activity, respectively, have not been studied in sarcopenic patients with HF. Therefore, the aim of this study was to evaluate the impact of autonomic modulation assessed by MSNA (by microneurography technique) and HRR immediately after maximal exercise testing in patients with HF and sarcopenia. 


\section{Methods}

\section{Study population}

Between May 1, 2016 and December 31, 2017, we prospectively enrolled 116 male outpatients with stable chronic HF. Inclusion criteria were: (1) age between 18 and 65 years old; (2) at least 1 year of HF diagnosis; (3) left ventricular ejection fraction (LVEF) lower than $40 \%$ measured by echocardiography; (4) non-ischemic and ischemic etiologies; (5) compensated HF with optimal medication for at least three months prior the study; and (6) New York Heart Association (NYHA) class I to IV.

Patients with autonomic diabetic neuropathy, chronic renal failure with haemodialysis, heart transplantation, pacemaker, muscular dystrophy (i.e. Duchenne muscular dystrophy), any hormonal treatment, history of cancer, ongoing infection, and myocardial infarction with percutaneous coronary intervention or revascularization up to 6 months prior to the study entry, were not included.

\section{Muscle sympathetic nerve activity}

MSNA was directly recorded from the peroneal nerve using the microneurography technique. ${ }^{15,16}$ Multiunit post-ganglionic muscle sympathetic nerve recordings were made using a tungsten microelectrode placed in the peroneal nerve near the fibular head. Nerve signals were amplified by a factor of 50,000 to 100,000 and band-pass filtered (700 to $2000 \mathrm{~Hz}$ ). For recording and analysis, nerve activity was rectified and integrated (time constant 0.1 second) to obtain a mean voltage display of sympathetic nerve activity. MSNA was expressed as burst frequency (bursts per minute).

\section{Maximal cardiopulmonary exercise test}

All patients underwent symptom-limited cardiopulmonary exercise test (Vmax Encore 29 System; VIASYS Healthcare Inc., Palm Springs, California, USA) performed on a cycle ergometer (Ergometer 800S; SensorMedics, Yorba Linda, California, USA), using a ramp protocol with workload increments of 5 or 10 Watts per minute. Oxygen consumption $\left(\mathrm{VO}_{2}\right)$ and carbon dioxide output $\left(\mathrm{VCO}_{2}\right)$ were measured by means of gas exchange on a breath-by-breath basis and expressed as 30-s averages. The patients were initially monitored for 2 minutes at rest when seated on the ergometer; then they were instructed to pedal at a pace of 60-70 rpm and the completion of the test occurred when, in spite of verbal encouragement, the patient reached maximal volitional fatigue. A respiratory exchange ratio (RER) higher than 1.10 was reached for all patients. Heart rate (HR) was monitored continuously at rest, during the test and recovery phase, using a 12-lead digital electrocardiogram (CardioSoft 6.51 ECG/CAM-14, GE Medical Systems Information Technologies, Wisconsin, USA). ${ }^{17}$

After achieving peak workload, the patients continued to pedal at 10 watts for 2 minutes, followed by 4 minutes seated on the ergometer, this 6-min period was considered the recovery phase. Delta $(\Delta) \mathrm{HRR}$ was calculated by subtracting the $H R$ values at $1^{\text {st }}(\triangle H R R 1)$ and $2^{\text {nd }}(\triangle H R R 2)$ minutes of the recovery phase from the peak HR. ${ }^{12}$

\section{Body composition and muscle strength}

Body composition measurements - total lean and fat mass - were performed using dual-energy X-ray absorptiometry (DXA) (Lunar iDXA; GE Medical Systems Lunar, Madison, USA). Then, skeletal muscle mass index (SMI) was calculated as the sum of appendicular lean muscle mass of both arms and legs divided by height in meters squared. ${ }^{18}$

After adjusting handle position, muscle strength was assessed by handgrip dynamometer (Model J00105; Jamar Hydraulic Hand Dynamometer) using the dominant hand in a supinated position with elbow flexed at $90^{\circ}$. There was 1-min rest interval between efforts and the maximum value of three attempts was used. ${ }^{19}$

Sarcopenia was defined as SMI and muscle strength lower than $7.26 \mathrm{~kg} / \mathrm{m}^{2}$ and $30 \mathrm{~kg}$, respectively. ${ }^{20}$

\section{Laboratory Measurements}

Blood samples were drawn in the morning after $12 \mathrm{~h}$ overnight fasting. The laboratory tests included B-type natriuretic peptide (BNP; $\mathrm{pg} / \mathrm{mL}$ ) plasma level, serum sodium $(\mathrm{mEq} / \mathrm{L})$, serum potassium $(\mathrm{mEq} / \mathrm{L})$, creatinine $(\mathrm{mg} / \mathrm{dL})$, haemoglobin level $(\mathrm{g} / \mathrm{dL})$, high-sensitivity C-reactive protein (hs-CRP; mg/L), lipid profile (triglyceride, total cholesterol, high-density lipoprotein, and low-density lipoprotein; mg/dL), and fasting glucose $(\mathrm{mg} / \mathrm{dL})$.

\section{Statistical analysis}

Data are presented as mean \pm standard deviation and median with lower and upper quartile $(95 \% \mathrm{Cl})$. One-sample Kolmogorov-Smirnov test was used to evaluate the distribution normality of the studied variables. Student's t-test and Mann-Whitney $U$ test were used to compare parametric and nonparametric variables, respectively. Chi-square test and Spearman's correlation were used as appropriate. The Statistical Package for the Social Sciences version 23 (SPSS Inc., Chicago, Illinois, USA) was used to perform all the statistical analysis. $P$ value lower than 0.05 was considered statistically significant.

\section{Results}

\section{Clinical-demographic data}

We prospectively enrolled 116 male patients (Table 1) with stable chronic HF, 33 of whom were identified to have sarcopenia (28\%). Patients with sarcopenia were older, had higher BNP concentration, and lower hemoglobin compared with patients without sarcopenia. No difference was found between sarcopenic and non-sarcopenic patients regarding the dosage of $\beta$-blocker medication $(20 \pm 9.6$ vs. $23 \pm 10.5$ mg b.i.d., $p=0.39$; respectively) and medication in general (Table 1).

Muscle sympathetic nerve activity, heart rate recovery and functional capacity

Patients with sarcopenia had higher MSNA (Figure 1) and lower $\triangle H R R 1$ and $\triangle H R R 2$ (Figure 2) when compared with non-sarcopenic patients. There was no statistical difference in resting HR and peak HR between sarcopenic and nonsarcopenic patients. 


\section{Original Article}

Table 1 - Demographic and clinical characteristics of the study population

\begin{tabular}{|c|c|c|c|c|}
\hline Variables & All patients $(n=116)$ & Patients with sarcopenia $(n=33$ ) & Patients without sarcopenia $(n=83)$ & $P$ value \\
\hline Age (y) & $55 \pm 9$ & $59 \pm 6$ & $54 \pm 9$ & 0.002 \\
\hline Weight (kg) & $71.1 \pm 14.4$ & $59.4 \pm 7.4$ & $75.8 \pm 13.8$ & $<0.001$ \\
\hline Height (m) & $1.67 \pm 0.07$ & $1.66 \pm 0.07$ & $1.67 \pm 0.07$ & 0.401 \\
\hline $\operatorname{BMl}\left(\mathrm{kg} / \mathrm{m}^{2}\right)$ & $25.5 \pm 4.5$ & $21.6 \pm 2.5$ & $27.1 \pm 4.2$ & $<0.001$ \\
\hline Aetiology (Ischaemic/non-ischaemic) & $30 / 86$ & $8 / 25$ & $22 / 61$ & 1.000 \\
\hline NYHA class (I/II/III/IV) & $40 / 41 / 28 / 7$ & $9 / 11 / 11 / 2$ & $31 / 30 / 17 / 5$ & 0.500 \\
\hline $\operatorname{LVEF}(\%)$ & $28 \pm 8$ & $26 \pm 7$ & $29 \pm 8$ & 0.124 \\
\hline $\mathrm{BNP}(\mathrm{pg} / \mathrm{mL})$ & $773 \pm 877$ & $1159 \pm 924$ & $621 \pm 816$ & 0.006 \\
\hline Sodium (mEq/L) & $139 \pm 3$ & $138 \pm 4$ & $139 \pm 3$ & 0.383 \\
\hline Potassium (mEq/L) & $4.6 \pm 0.4$ & $4.6 \pm 0.3$ & $4.6 \pm 0.4$ & 0.535 \\
\hline Creatinine (mg/dL) & $1.24 \pm 0.39$ & $1.27 \pm 0.47$ & $1.23 \pm 0.35$ & 0.568 \\
\hline Haemoglobin (g/dL) & $13.9 \pm 1.7$ & $13.3 \pm 1.6$ & $14.1 \pm 1.7$ & 0.022 \\
\hline hs-CRP (mg/L) & $8.96 \pm 16.0$ & $12.4 \pm 13.6$ & $7.6 \pm 16.7$ & 0.147 \\
\hline Triglyceride (mg/dL) & $118 \pm 68$ & $96 \pm 38$ & $127 \pm 75$ & 0.031 \\
\hline Cholesterol (mg/dL) & $170 \pm 45$ & $159 \pm 37$ & $174 \pm 48$ & 0.111 \\
\hline $\mathrm{HDL}(\mathrm{mg} / \mathrm{dL})$ & $44 \pm 15$ & $47 \pm 16$ & $44 \pm 14$ & 0.306 \\
\hline $\mathrm{LDL}(\mathrm{mg} / \mathrm{dL})$ & $103 \pm 35$ & $95 \pm 25$ & $106 \pm 38$ & 0.155 \\
\hline Fasting glucose (mg/dL) & $108 \pm 21$ & $106 \pm 24$ & $109 \pm 20$ & 0.510 \\
\hline \multicolumn{5}{|l|}{ Medication } \\
\hline$\beta$-blocker & & $33(100)$ & $78(94)$ & 0.319 \\
\hline Statins & & $18(55)$ & $49(59)$ & 0.682 \\
\hline ACEI/ARB & & $31(94)$ & $76(92)$ & 1.000 \\
\hline Diuretics & & $26(79)$ & $62(75)$ & 0.811 \\
\hline Anticoagulants & & $12(36)$ & $32(39)$ & 1.000 \\
\hline Hydralazine & & $6(18)$ & $18(22)$ & 0.802 \\
\hline Isosorbide & & $6(18)$ & $18(22)$ & 0.802 \\
\hline Spironolactone & & $24(73)$ & $58(70)$ & 0.824 \\
\hline
\end{tabular}

Data are presented as mean $\pm S D$ or \%. P value referred to Student's t-test and Chi-square test for medication. ACEl, angiotensin converting enzyme inhibitors: ARB: angiotensin receptor blockers; BMI: body mass index; BNP: B-type natriuretic peptide; HDL: high-density lipoprotein; $h s$-CRP: high-sensitivity C-reactive protein; LDL: low-density lipoprotein; LVEF: left ventricular ejection fraction; NYHA: New York Heart Association.

Spearman's correlation showed a positive correlation between appendicular lean muscle mass and $\triangle H R R 1$ and $\triangle$ HRR2 (Figures $3 \mathrm{~A}$ and $3 \mathrm{~B}$, respectively). In addition, we observed a negative correlation between appendicular lean muscle mass and MSNA (Figure 3C).

Absolute $\mathrm{VO}_{2 \text { peak' }}$, relative $\mathrm{VO}_{2 \text { peak' }}$ and peak workload were significantly lower in patients with sarcopenia than those without. Sarcopenic patients also showed higher ventilatory equivalent for carbon dioxide $\left(\mathrm{VE} / \mathrm{VCO}_{2}\right)$ slope and dead space to tidal volume (VD/VT $\mathrm{Teak}_{\text {) }}$ than non-sarcopenic patients, whereas $\mathrm{VE}_{\text {peak }}$ was lower in patients with sarcopenia than those without (Table 2).

\section{Body composition and muscle strength characteristics}

Body mass index was lower in sarcopenic patients when compared with non-sarcopenic, with a significant reduction in appendicular lean muscle mass, total lean mass, fat mass, and fat percentage (Table 2). SMI and muscle strength assessed by handgrip dynamometer were also lower in patients with sarcopenia compared with those without sarcopenia.

\section{Discussion}

The main and new findings of this study are that sarcopenic patients with HF have increased resting MSNA and blunted vagal reactivation after maximal exercise testing when compared with patients without sarcopenia. Moreover, the appendicular lean muscle mass seems to be associated with higher MSNA and blunted HRR. Additionally, as previously demonstrated, ${ }^{2}$ we also confirmed the reduction in exercise tolerance (decreased peak $\mathrm{VO}_{2}$ and peak workload) in patients with HF and muscle wasting. 


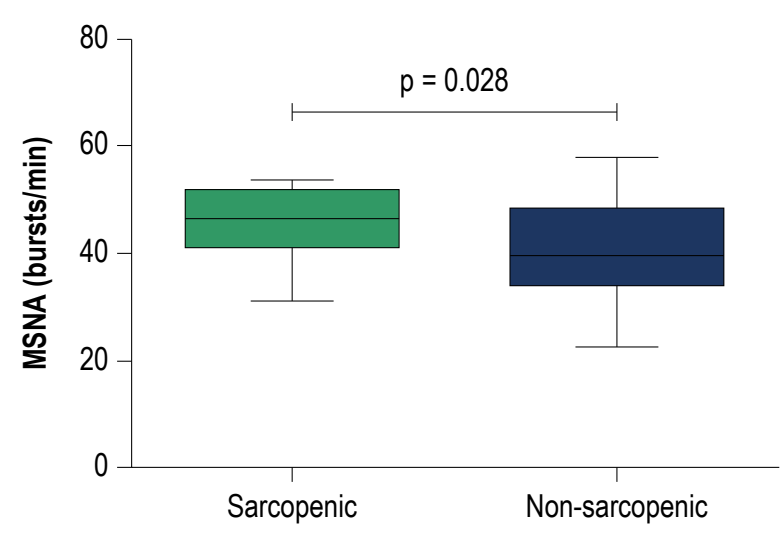

Figure 1 - Muscle sympathetic nerve activity (MSNA) in bursts/min. Values are presented as medians with lower and upper quartiles (Cl 95\%). Note that sarcopenic patients showed an increase of $18 \%$ in MSNA.

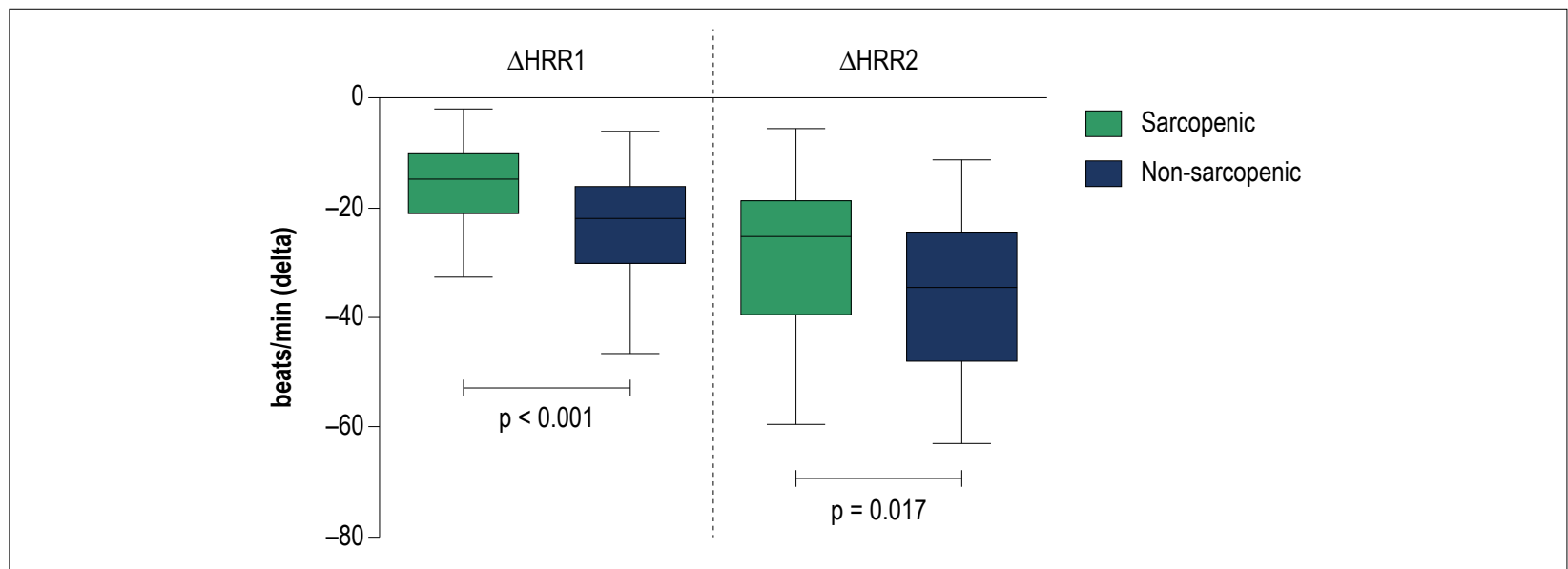

Figure 2 - Delta heart rate recovery at $1 s t(\Delta H R R 1)$ and 2nd ( $\triangle H R R 2)$ minutes immediately after maximal exercise testing. Values are presented as medians with lower and upper quartiles (Cl 95\%). Note that sarcopenic patients showed a lower HRR at 1 st (47\% difference) and 2nd minutes (40\% difference).

HF is a complex disease associated with several comorbidities. One of the major co-morbidities observed in patients with advanced chronic HF is sarcopenia, which is associated with poor prognosis. ${ }^{21}$ Although the aetiology of sarcopenia is multifactorial, several mechanisms have been suggested to explain this decrease in muscle mass in patients with $\mathrm{HF}$, such as increased inflammatory profile, ${ }^{22}$ increased oxidative stress, ${ }^{23}$ overactivation of ubiquitin-proteasome system, ${ }^{24}$ and increased C-terminal agrin fragment (CAF). ${ }^{25}$ These alterations, acting independently or in combination, may lead to excessive muscle protein degradation and reduced muscle protein synthesis.

Besides the mechanisms mentioned above, exacerbated sympathetic nerve activity seems to be an important pathophysiological feature in HF leading to the loss of skeletal muscle. ${ }^{6}$ In an experimental model of HF, Bacurau and colleagues ${ }^{6}$ showed that sympathetic hyperactivity contributes to the development of skeletal myopathy by changing muscle morphology. ${ }^{6} \beta_{2}$-adrenoceptors play a key role in regulating skeletal muscle mass in both anabolic and catabolic state. ${ }^{26}$
However, chronic sympathetic hyperactivity may be toxic to skeletal muscle, ${ }^{27}$ which favors weight loss and sarcopenia in patients with HF. Moreover, increased sympathetic outflow is associated with higher chance of arrhythmias, ${ }^{28}$ and adverse remodeling of the heart. ${ }^{29}$

Interestingly, pharmacological treatment of HF is focused on blocking sympathetic activity, mainly by using cardio-selective and non-selective $\beta$-blockers. ${ }^{30}$ Treatment with $\beta$-blockers can increase total body fat mass and total body fat content in patients with HF, without apparent improvement in muscle mass. ${ }^{30,31}$ In this study, we did not observe differences between groups in $\beta$-blocker treatment and dosage. In this context, future randomized clinical trials are required to assess the real impact of $\beta$-blocker therapy on skeletal muscle mass in patients with HF.

Previous studies showed that HRR has an important prognostic value in the general population ${ }^{12}$ and in patients with $\mathrm{HF}^{32}$ In addition, HRR is a very simple and easy way to indirectly evaluate the reactivation of the parasympathetic 


\section{Original Article}

A

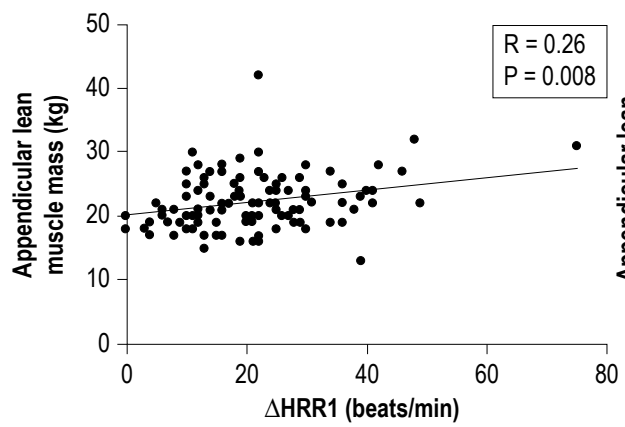

C
B

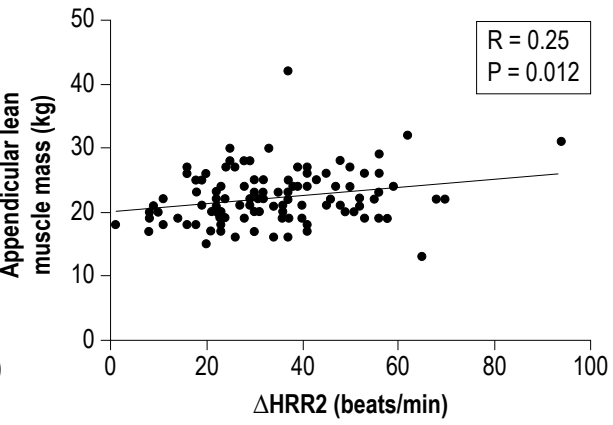

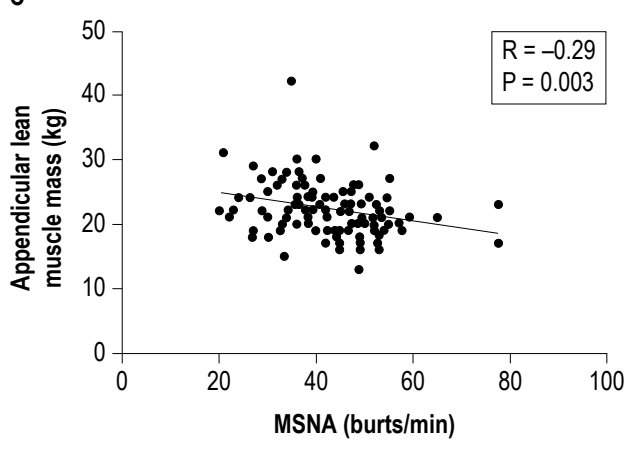

Figure 3 - (A) Spearman's correlation between appendicular lean muscle mass and delta heart rate recovery at 1st minute ( $\Delta H R R 1$ ). (B) Spearman's correlation between appendicular lean muscle mass and delta heart rate recovery at 2nd minute ( $\triangle H R R 2)$. (C) Spearman's correlation between appendicular lean muscle mass and muscle sympathetic nerve activity (MSNA).

Table 2 - Cardiopulmonary, body composition and strength variables of the patients

\begin{tabular}{|c|c|c|c|c|}
\hline Variables & All patients $(n=116)$ & Patients with sarcopenia $(n=33$ ) & Patients without sarcopenia $(n=83)$ & $P$ value \\
\hline Absolute $\mathrm{VO}_{2 \text { peak }}(\mathrm{L} / \mathrm{min})$ & $1.43 \pm 0.48$ & $1.09 \pm 0.31$ & $1.58 \pm 0.47$ & $<0.001$ \\
\hline Relative $\mathrm{VO}_{\text {2peak }}(\mathrm{ml} /(\mathrm{kg} / \mathrm{min}))$ & $20.1 \pm 6.3$ & $18.3 \pm 5.3$ & $21.0 \pm 6.5$ & 0.036 \\
\hline Peak workload (Watts) & $103 \pm 47$ & $77 \pm 27$ & $115 \pm 51$ & $<0.001$ \\
\hline Resting HR (beats/min) & $72 \pm 13$ & $75 \pm 16$ & $71 \pm 12$ & 0.254 \\
\hline $\mathrm{HR}_{\text {peak }}($ beats/min $)$ & $134 \pm 27$ & $130 \pm 27$ & $135 \pm 28$ & 0.323 \\
\hline $\mathrm{VE} / \mathrm{VCO}_{2}$ slope & $35 \pm 7$ & $37 \pm 8$ & $33 \pm 7$ & 0.015 \\
\hline$V D / N T_{\text {peak }}$ & $0.17 \pm 0.02$ & $0.19 \pm 0.04$ & $0.16 \pm 0.04$ & $<0.001$ \\
\hline $\mathrm{VE}_{\text {peak }}(\mathrm{L} / \mathrm{min})$ & $62.5 \pm 18.3$ & $53.5 \pm 14.1$ & $66.4 \pm 18.4$ & $<0.001$ \\
\hline \multicolumn{5}{|c|}{ Body composition and strength } \\
\hline Total LM (kg) & $49.6 \pm 8.4$ & $43.0 \pm 5.2$ & $52.5 \pm 8.0$ & $<0.001$ \\
\hline ALM (kg) & $22.2 \pm 4.3$ & $18.3 \pm 2.3$ & $23.9 \pm 3.8$ & $<0.001$ \\
\hline $\mathrm{SMI}\left(\mathrm{kg} / \mathrm{m}^{2}\right)$ & $7.97 \pm 1.21$ & $6.63 \pm 0.58$ & $8.54 \pm 0.92$ & $<0.001$ \\
\hline Fat mass $(\mathrm{kg})$ & $18.2 \pm 8.5$ & $12.9 \pm 4.9$ & $20.5 \pm 8.8$ & $<0.001$ \\
\hline Fat $(\%)$ & $26 \pm 8$ & $22 \pm 7$ & $27 \pm 8$ & $<0.001$ \\
\hline Handgrip strength (kg) & $33 \pm 8$ & $26 \pm 3$ & $36 \pm 8$ & $<0.001$ \\
\hline
\end{tabular}

Data are presented as mean \pm standard deviation or \%. P value referred to Student's t-test. ALM: appendicular lean muscle mass; HR: heart rate; LM: lean mass; SMI: skeletal muscle mass index; VE: ventilation; $\mathrm{VE}_{\mathrm{NCO}}$ : ventilatory equivalent for carbon dioxide; $\mathrm{VDNTT}$ : dead space to tidal volume; $\mathrm{VO}_{2}$ : oxygen consumption. 
nervous system immediately after maximum effort in cardiopulmonary exercise testing. ${ }^{11}$ Several investigators showed that the kinetic of HRR in a 6-min recovery period was reduced in patients with $\mathrm{HF}^{33}$ and this reduction seems to be independent of $\beta$-adrenergic blocker therapy. ${ }^{34}$ Ushijima et al. ${ }^{35}$ showed an association between norepinephrine and HRR in patients with myocardial infarction, arguing that increased sympathetic excitation at maximum exercise may suppress the parasympathetic reactivation leading to HRR attenuation. ${ }^{35}$

Taken together, the sympathovagal impairment in patients with $\mathrm{HF}$ is associated with poor outcome, and this autonomic imbalance may worsen the loss of muscle mass in these patients. In fact, we showed greater MSNA and lower decrease in HRR at $1^{\text {st }}$ and $2^{\text {nd }}$ minutes post-exercise in sarcopenic patients with HF. Furthermore, reduced appendicular lean muscle mass was correlated with lower HRR1 ( $r=0.26)$, HRR2 $(r=0.25)$ and greater MSNA $(r=-0.29)$.

We recognize limitations in our study. The present study included only male patients, so we are not able to generalize these results to female patients with HF. Further studies are necessary to investigate the influence of sarcopenia on gender-related differences. The date when HF was diagnosed was not available in patients' medical records, and to compensate for this missing information, we included only patients with at least one year of diagnosis. We assessed parasympathetic activity using the HRR as a marker of vagal reactivation. Although our study has a clinical applicability, more studies using HR variability should clarify the role of cardiac autonomic control on sarcopenia in patients with HF.

\section{Conclusion}

Sympatho-vagal imbalance seems to be associated with sarcopenia in male patients with HF. These results highlight the importance of a therapeutic approach in patients with muscle wasting and increased peripheral sympathetic outflow.

\section{References}

1. von Haehling S, Ebner N, Dos Santos MR, Springer J, Anker SD. Muscle wasting and cachexia in heart failure: mechanisms and therapies. Nat Rev Cardiol. 2017;14(6):323-41.

2. Fulster S, Tacke M, Sandek A, Ebner N, Tschope C, Doehner W, et al. Muscle wasting in patients with chronic heart failure: results from the studies investigating co-morbidities aggravating heart failure (SICA-HF). Eur Heart J. $2013 ; 34(7): 512-9$

3. Dos Santos MR, Saitoh M, Ebner N, Valentova M, Konishi M, Ishida J, et al. Sarcopenia and Endothelial Function in Patients With Chronic Heart Failure: Results From the Studies Investigating Comorbidities Aggravating Heart Failure (SICA-HF). J Am Med Dir Assoc. 2017;18(3):240-5.

4. Hajahmadi M, Shemshadi S, Khalilipur E, Amin A, Taghavi S, Maleki M, et al. Muscle wasting in young patients with dilated cardiomyopathy. J Cachexia Sarcopenia Muscle. 2017;8(4):542-8.

\section{Author contributions}

Conception and design of the research: Fonseca GWP, dos Santos MR, Alves MJNN; Acquisition of data: Fonseca GWP, dos Santos MR, Souza FR, Costa MJA, Takayama L, Pereira RMR, Alves MJNN; Analysis and interpretation of the data: Fonseca GWP, dos Santos MR, Costa MJA,Souza FR, Pereira RMR, Negrão CE, Alves MJNN; Statistical analysis: Fonseca GWP, dos Santos MR; Obtaining financing: Negrão CE, Alves MJNN; Writing of the manuscript: Fonseca GWP, Souza FR, Alves MJNN; Critical revision of the manuscript for intellectual content: dos Santos MR, von Haehling $S$, Pereira RMR, Negrão CE, Anker SD, Alves MJNN.

\section{Potential Conflict of Interest}

No potential conflict of interest relevant to this article was reported.

\section{Sources of Funding}

This study was funded by Fundação de Amparo à Pesquisa do Estado de São Paulo (FAPESP; 2015/22814-5). Fonseca GWP was supported by Conselho Nacional de Desenvolvimento Científico e Tecnológico (CNPq; 148758/2016-9); Dos Santos MR by FAPESP (2016/24306-0); Negrão CE by FAPESP (2015/22814-5). All foundations are from São Paulo-SP, Brazil.

\section{Study Association}

This article is part of the thesis of Doctoral submitted by Guilherme Wesley Peixoto da Fonseca, from Universidade Federal de São Paulo.

\section{Ethics approval and consent to participate}

This study was approved by the Ethics Committee of the CAPPesq under the protocol number 0892/07. All the procedures in this study were in accordance with the 1975 Helsinki Declaration, updated in 2013. Informed consent was obtained from all participants included in the study.

5. Barretto AC, Santos AC, Munhoz R, Rondon MU, Franco FG, Trombetta IC, et al. Increased muscle sympathetic nerve activity predicts mortality in heart failure patients. Int J Cardiol. 2009;135(3):302-7.

6. Bacurau AV, Jardim MA, Ferreira JC, Bechara LR, Bueno CR Jr., Alba-Loureiro TC, et al. Sympathetic hyperactivity differentially affects skeletal muscle mass in developing heart failure: role of exercise training. J Appl Physiol (1985). 2009;106(5):1631-40.

7. Kim YS, Sainz RD, Summers RJ, Molenaar P. Cimaterol reduces beta-adrenergic receptor density in rat skeletal muscles. J Anim Sci. 1992;70(1):115-22

8. Casolo G, Balli E, Taddei T, Amuhasi J, Gori C. Decreased spontaneous heart rate variability in congestive heart failure. Am J Cardiol. 1989;64(18):1162-7.

9. De Jong MJ, Randall DC. Heart rate variability analysis in the assessment of autonomic function in heart failure. J Cardiovasc Nurs. 2005;20(3):186-95. 


\section{Original Article}

10. Binkley PF, Nunziata E, Haas GJ, Nelson SD, Cody RJ. Parasympathetic withdrawal is an integral component of autonomic imbalance in congestive heart failure: demonstration in human subjects and verification in a paced canine model of ventricular failure. J Am Coll Cardiol. 1991;18(2):464-72.

11. Shetler K, Marcus R, Froelicher VF, Vora S, Kalisetti D, Prakash M, et al. Heart rate recovery: validation and methodologic issues. J Am Coll Cardiol. 2001;38(7):1980-7.

12. Cole CR, Blackstone EH, Pashkow FJ, Snader CE, Lauer MS. Heart-rate recovery immediately after exercise as a predictor of mortality. N Engl J Med. 1999;341(18):1351-7.

13. Arena R, Guazzi M, Myers J, Peberdy MA. Prognostic value of heart rate recovery in patients with heart failure. Am Heart J. 2006;151(4):851 e7-13.

14. Watanabe J, Thamilarasan M, Blackstone EH, Thomas JD, Lauer MS. Heart rate recovery immediately after treadmill exercise and left ventricular systolic dysfunction as predictors of mortality: the case of stress echocardiography. Circulation. 2001;104(16):1911-6.

15. Vallbo AB, Hagbarth KE, Torebjork HE, Wallin BG. Somatosensory, proprioceptive, and sympathetic activity in human peripheral nerves. Physiol Rev. 1979;59(4):919-57.

16. Delius W, Hagbarth KE, Hongell A, Wallin BG. Manoeuvres affecting sympathetic outflow in human muscle nerves. Acta Physiol Scand. 1972;84(1):82-94.

17. Dos Santos MR, Sayegh AL, Bacurau AV, Arap MA, Brum PC, Pereira RM, et al. Effect of Exercise Training and Testosterone Replacement on Skeletal Muscle Wasting in Patients With Heart Failure With Testosterone Deficiency. Mayo Clin Proc. 2016;91(5):575-86.

18. Baumgartner RN, Koehler KM, Gallagher D, Romero L, Heymsfield SB, Ross RR, et al. Epidemiology of sarcopenia among the elderly in New Mexico. Am J Epidemiol. 1998;147(8):755-63.

19. Roberts HC, Denison HJ, Martin HJ, Patel HP, Syddall H, Cooper C, et al. A review of the measurement of grip strength in clinical and epidemiological studies: towards a standardised approach. Age Ageing. $2011 ; 40(4): 423-9$

20. Cruz-Jentoft AJ, Baeyens JP, Bauer JM, Boirie Y, Cederholm T, Landi F, et al. Sarcopenia: European consensus on definition and diagnosis: Report of the European Working Group on Sarcopenia in Older People. Age Ageing. 2010;39(4):412-23.

21. Narumi T, Watanabe T, Kadowaki S, Takahashi T, Yokoyama M, Kinoshita D, et al. Sarcopenia evaluated by fat-free mass index is an important prognostic factor in patients with chronic heart failure. Eur J Intern Med. 2015;26(2):118-22.
22. Janssen SP, Gayan-Ramirez G, Van den Bergh A, Herijgers P, Maes K, Verbeken $\mathrm{E}$, et al. Interleukin- 6 causes myocardial failure and skeletal muscle atrophy in rats. Circulation. 2005;111(8):996-1005.

23. Bechara LR, Moreira JB, Jannig PR, Voltarelli VA, Dourado PM, Vasconcelos $A R$, et al. NADPH oxidase hyperactivity induces plantaris atrophy in heart failure rats. Int J Cardiol. 2014;175(3):499-507.

24. Li YP, Chen Y, Li AS, Reid MB. Hydrogen peroxide stimulates ubiquitinconjugating activity and expression of genes for specific E2 and E3 proteins in skeletal muscle myotubes. Am J Physiol Cell Physiol. 2003;285(4):C806-12.

25. Steinbeck L, Ebner N, Valentova M, Bekfani T, Elsner S, Dahinden P, et al. Detection of muscle wasting in patients with chronic heart failure using C-terminal agrin fragment: results from the Studies Investigating Co-morbidities Aggravating Heart Failure (SICA-HF). Eur J Heart Fail. 2015;17(12):1283-93.

26. Kim YS, Sainz RD. Beta-adrenergic agonists and hypertrophy of skeletal muscles. Life Sci. 1992;50(6):397-407.

27. Lymperopoulos A, Rengo G, Koch WJ. Adrenergic nervous system in heart failure: pathophysiology and therapy. Circ Res. 2013;113(6):739-53.

28. Volders PG. Novel insights into the role of the sympathetic nervous system in cardiac arrhythmogenesis. Heart Rhythm. 2010;7(12):1900-6.

29. Machackova J, Sanganalmath SK, Barta J, Dhalla KS, Dhalla NS. Amelioration of cardiac remodeling in congestive heart failure by beta-adrenoceptor blockade is associated with depression in sympathetic activity. Cardiovasc Toxicol. 2010;10(1):9-16.

30. Lainscak M, Keber I, Anker SD. Body composition changes in patients with systolic heart failure treated with beta blockers: a pilot study. Int J Cardiol. 2006;106(3):319-22.

31. Cvan Trobec K, Grabnar I, Kerec Kos M, Vovk T, Trontelj J, Anker SD, et al. Bisoprolol pharmacokinetics and body composition in patients with chronic heart failure: a longitudinal study. Eur J Clin Pharmacol. 2016;72(7):813-22.

32. Nanas S, Anastasiou-Nana M, Dimopoulos S, Sakellariou D, Alexopoulos G, Kapsimalakou S, et al. Early heart rate recovery after exercise predicts mortality in patients with chronic heart failure. Int J Cardiol. 2006;110(3):393-400.

33. Myers J, Hadley D, Oswald U, Bruner K, Kottman W, Hsu L, et al. Effects of exercise training on heart rate recovery in patients with chronic heart failure. Am Heart J. 2007;153(6):1056-63.

34. Racine N, Blanchet M, Ducharme A, Marquis J, Boucher JM, Juneau M, et al. Decreased heart rate recovery after exercise in patients with congestive heart failure: effect of beta-blocker therapy. J Card Fail. 2003;9(4):296-302.

35. Ushijima A, Fukuma N, Kato Y, Aisu N, Mizuno K. Sympathetic excitation during exercise as a cause of attenuated heart rate recovery in patients with myocardial infarction. J Nippon Med Sch. 2009;76(2):76-83. 


\title{
Muscle mass, muscle strength, and functional capacity in patients with heart failure of Chagas disease and other aetiologies
}

\author{
Guilherme Wesley Peixoto da Fonseca ${ }^{1,2,3}$ D , Tania Garfias Macedo ${ }^{2,3}$, Nicole Ebner ${ }^{2,3}$, Marcelo Rodrigues \\ dos Santos ${ }^{1}$, Francis Ribeiro de Souza ${ }^{1}$, Charles Mady ${ }^{1}$, Liliam Takayama ${ }^{4}$, Rosa Maria Rodrigues Pereira ${ }^{4}$, \\ Wolfram Doehner ${ }^{5,6}$, Stefan D. Anker ${ }^{5,6}$, Carlos Eduardo Negrão ${ }^{1,7}$, Maria Janieire de Nazaré Nunes Alves ${ }^{1}$ \\ and Stephan von Haehling $2,3 *$
}

${ }^{1}$ Heart Institute (InCor), University of São Paulo Medical School, São Paulo, Brazil; ${ }^{2}$ Department of Cardiology and Pneumology, University of Göttingen Medical Center (UMG), Robert-Koch-Strasse 40, Göttingen, D - 37075, Germany; ${ }^{3}$ German Centre for Cardiovascular Research (DZHK) partner site Göttingen, Göttingen, Germany; ${ }^{4}$ Bone Metabolism Laboratory, Rheumatology Division, University of São Paulo Medical School, São Paulo, Brazil; ${ }^{5}$ Department of Cardiology (CVK) and Berlin Institute of Health Center for Regenerative Therapies (BCRT), Charité - Universitätsmedizin Berlin, Berlin, Germany; ${ }^{6}$ German Centre for Cardiovascular Research (DZHK) partner site Berlin, Charité - Universitätsmedizin Berlin, Berlin, Germany; ${ }^{7}$ School of Physical Education and Sports, University of São Paulo, São Paulo, Brazil

\section{Abstract}

Aims Patients with Chagas disease and heart failure (HF) have a poor prognosis similar to that of patients with ischaemic or dilated cardiomyopathy. However, the impact of body composition and muscle strength changes in these aetiologies is still unknown. We aimed to evaluate these parameters across aetiologies in two distinct cohort studies [TESTOsterone-Heart Failure trial (TESTO-HF; Brazil) and Studies Investigating Co-morbidities Aggravating Heart Failure (SICA-HF; Germany)].

Methods and results A total of 64 male patients with left ventricular ejection fraction $\leq 40 \%$ were matched for body mass index and New York Heart Association class, including 22 patients with Chagas disease (TESTO-HF; Brazil), and 20 patients with dilated cardiomyopathy and 22 patients with ischaemic heart disease (SICA-HF; Germany). Lean body mass (LBM), appendicular lean mass (ALM), and fat mass were assessed by dual energy X-ray absorptiometry. Sarcopenia was defined as ALM divided by height in metres squared $<7.0 \mathrm{~kg} / \mathrm{m}^{2}$ (ALM/height ${ }^{2}$ ) and handgrip strength cut-off for men according to the European Working Group on Sarcopenia in Older People. All patients performed maximal cardiopulmonary exercise testing. Forearm blood flow (FBF) was measured by venous occlusion plethysmography. Chagasic and ischaemic patients had lower total fat mass $(16.3 \pm 8.1$ vs. $19.3 \pm 8.0$ vs. $27.6 \pm 9.4 \mathrm{~kg} ; P<0.05)$ and reduced peak oxygen consumption $\left(\mathrm{VO}_{2}\right)$ $(1.17 \pm 0.36$ vs. $1.15 \pm 0.36$ vs. $1.50 \pm 0.45 \mathrm{~L} / \mathrm{min} ; P<0.05)$ than patients with dilated cardiomyopathy, respectively. Chagasic patients showed a trend towards decreased LBM when compared with ischaemic patients (48.3 $\pm 7.6 \mathrm{vs}$. $54.2 \pm 6.3 \mathrm{~kg}$; $P=0.09)$. Chagasic patients showed lower handgrip strength $(27 \pm 8$ vs. $37 \pm 11$ vs. $36 \pm 14 \mathrm{~kg} ; P<0.05)$ and FBF (1.84 \pm 0.54 vs. $2.75 \pm 0.76$ vs. $3.42 \pm 1.21 \mathrm{~mL} / \mathrm{min} / 100 \mathrm{~mL} ; P<0.01)$ than ischaemic and dilated cardiomyopathy patients, respectively. There was no statistical difference in the distribution of sarcopenia between groups $(P=0.87)$. In addition, FBF correlated positively with LBM $(r=0.31 ; P=0.012)$, ALM $(r=0.25 ; P=0.046)$, and handgrip strength $(r=0.36$; $P=0.004$ ). In a logistic regression model using peak $\mathrm{VO}_{2}$ as the dependent variable, haemoglobin (odds ratio, $1.506 ; 95 \%$ confidence interval, 1.043-2.177; $P=0.029$ ) and ALM (odds ratio, 1.179; 95\% confidence interval, $1.011-1.374 ; P=0.035$ ) were independent predictors for peak $\mathrm{VO}_{2}$ adjusted by age, left ventricular ejection fraction, New York Heart Association, creatinine, and FBF.

Conclusions Patients with Chagas disease and HF have decreased fat mass and exhibit reduced peripheral blood flow and impaired muscle strength compared with ischaemic HF patients. In addition, patients with Chagas disease and HF show a tendency to have greater reduction in total LBM, with ALM remaining an independent predictor of reduced functional capacity in these patients. The percentage of patients affected by sarcopenia was equal between groups. 
*Correspondence to: Prof. Stephan von Haehling, MD PhD FESC FHFA, Department of Cardiology and Pneumology, University of Göttingen Medical Center (UMG), RobertKoch-Strasse 40, D - 37075 Göttingen, Germany. Tel: +49 55139 20911; Fax: +49 55139 20918. Email: stephan.von.haehling@web.de

\section{Introduction}

Heart failure (HF) is highly prevalent in Western countries, affecting 5.7 million patients in the USA alone and more than 37 million globally. ${ }^{1}$ The aetiology of $\mathrm{HF}$ is multifactorial; guidelines of the European Society of Cardiology ${ }^{2}$ state that many patients have several different pathologies-cardiovascular and non-cardiovascular-that conspire to cause HF. In general, more than two-thirds of all cases of HF can be attributed to four underlying conditions: ischaemic heart disease, chronic obstructive pulmonary disease, hypertensive heart disease, and rheumatic heart disease. ${ }^{1}$ Immune-mediated and inflammatory damage, frequently caused by bacteria, spirochaetes, fungi, protozoa, parasites, rickettsiae, and viruses, play a less important role in Western countries. However, parasites like Trypanosoma cruzi have become an extremely important consideration in the differential diagnosis of HF in certain regions of the world. Indeed, the prevalence of the zoonosis Chagas disease among patients with $\mathrm{HF}$ has been described to reach $22.7 \%$ in endemic regions in Latin America, especially in developing countries such as Brazil. ${ }^{3}$

Although Chagas disease has been commonly described as a feature of rural and socio-economically deprived communities, the disease has reached an urban and global proportion in recent decades as a result of migration patterns, impacting the epidemiological profile of the disease even in non-endemic regions such as the USA and Europe. ${ }^{4}$ In light of these alarming facts, it is noteworthy that patients with $\mathrm{HF}$ and Chagas disease have shown to have higher rates of hospitalization and mortality as compared with patients with ischaemic and other non-ischaemic causes of HF. ${ }^{3,5}$ Despite this, little is known about the metabolic profiles and the co-morbidity burden of patients with $\mathrm{HF}$ and Chagas disease. Treatment involves parasite-specific drugs (benznidazole and nifurtimox) during acute infection and times of reactivation and HF-specific drugs and devices. With regard to HF-specific drugs, a scarcity of data is related to the fact that most large-scale HF trials have excluded patients with infectious diseases like Chagas disease. However, beta-blocker therapy has-despite concerns about the appearance of bradyarrhythmiasproven to be safe and efficacious in patients with Chagas disease. ${ }^{6,7}$

Some data regarding metabolic and epidemiological features of patients with HF due to Chagas disease have been published. For instance, patients with Chagas disease-associated HF appear to have a lower body mass index (BMI) in comparison with patients with ischaemic or non-ischaemic $\mathrm{HF}^{3}$ and, when only patients with Chagas disease and $\mathrm{HF}$ are considered, those who died had lower values of $\mathrm{BMI}$ as well. ${ }^{8}$ Additionally, reduced body weight has also been reported in Chagas disease patients with HF compared with their counterparts with Chagas disease alone. ${ }^{9}$ Thus, it appears that an overlap is present between Chagas disease and the development/progression of HF that leads to alterations in body weight. Unfortunately, there are no studies assessing body composition features in these patients, neither in a cross-sectional nor longitudinal fashion.

The aim of this study was to evaluate body composition, muscle mass, muscle strength, and functional capacity in patients with Chagas disease enrolled into the Brazilian TESTOsterone-Heart Failure trial (TESTO-HF) and to compare them with data from patients with ischaemic or non-ischaemic HF enrolled into the Studies Investigating Co-morbidities Aggravating Heart Failure (SICA-HF) enrolled in Germany.

\section{Material and methods}

\section{Study population}

Patients for this analysis were selected from the TESTO-HF trial and the SICA-HF study. Of the total population enrolled in Brazil (169 patients; TESTO-HF) and Germany (327 patients; SICA-HF), 64 male patients were matched by BMI with a tolerance of 2 standard deviation and New York Heart Association (NYHA) functional class II and III. In Germany, patients were enrolled between March 2010 and April 2015 at Charité Medical School in Berlin. In Brazil, patients were enrolled between May 2016 and March 2019 at the Heart Institute of the University of São Paulo Medical School. The inclusion criteria for both studies have been described in detail elsewhere ${ }^{10,11}$ (NCT01872299; NCT03463226). For the present analysis, only patients were eligible if they were older than 18 years and had clinical signs and symptoms of chronic HF (NYHA II and III) and a left ventricular ejection fraction (LVEF) $\leq 40 \%$.

Patients with heart transplantation, muscular dystrophy (i.e. Duchenne muscular dystrophy), stroke, and myocardial infarction with percutaneous coronary intervention or revascularization within at least 6 weeks prior to the study entry were excluded. All patients provided written informed consent before any study-related procedure in both centres. The studies were approved by the local ethics committees in both locations. 


\section{Body composition and muscle strength assessment}

Dual energy X-ray absorptiometry (DXA) was performed to measure body composition in patients from Brazil (Lunar iDXA; GE Medical Systems Lunar, Madison, WI, USA) and Germany (GE Medical Systems, Madison, WI, USA). An experienced technician in each location performed DXA measurements. The measurements included total and regional fat mass, bone mass, and lean body mass (LBM). Appendicular lean mass (ALM), that is the lean mass of the arms and legs combined, was calculated as the sum of lean mass in the upper and lower limbs. Sarcopenia was defined as ALM divided by height in metres squared $<7.0 \mathrm{~kg} / \mathrm{m}^{2}$ (ALM/height ${ }^{2}$ ) and handgrip strength cut-off for men proposed by the European Working Group on Sarcopenia in Older People. ${ }^{12}$

Muscle strength was assessed by handgrip dynamometer (Jamar Hydraulic Hand Dynamometer, model J00105 in Brazil and Saehan Corporation Korea Hydraulic Hand Dynamometer, model SH5001 in Germany), and the highest value of three attempts was used.

\section{Cardiopulmonary exercise test}

All patients underwent a symptom-limited cardiopulmonary exercise test in both centres, using a ramp protocol. Metabolic parameters, including oxygen consumption $\left(\mathrm{VO}_{2}\right.$ in $\left.\mathrm{mL}\right)$ and carbon dioxide output $\left(\mathrm{VCO}_{2}\right.$ in $\left.\mathrm{mL}\right)$, were collected by ventilation gases on a breath-by-breath basis. The test was considered maximal when a respiratory exchange ratio higher than 1.10 was reached.

However, patients in Brazil were assessed on a cycle ergometer while patients in Germany performed the cardiopulmonary exercise test on a treadmill. Moreover, studies have shown a difference of $10-20 \%$ in absolute peak $\mathrm{VO}_{2}$ between cycle ergometer and treadmill performance, with the higher values being reported in patients performing treadmill test. ${ }^{13}$ Thus, to adjust for these differences, we corrected the peak $\mathrm{VO}_{2}$ value using data from the well-designed study by Kim et al., which showed a mean difference of $16 \%$ in peak $\mathrm{VO}_{2}$ of patients with HF between these two modalities of exercise, demonstrating a linear increase in peak $\mathrm{VO}_{2}$ with a strong positive correlation $(r=0.92 ; P<0.001) .{ }^{14}$ Other studies have shown similar results between these exercise modalities in patients with HF. ${ }^{15-17}$

\section{Venous occlusion plethysmography}

Forearm blood flow (FBF) was measured by venous occlusion plethysmography. Briefly, this analysis was performed by placing a mercury-filled silastic tube around the forearm with a distance of $5 \mathrm{~cm}$ from the humeral-radial joint. The silastic was connected to a transducer that was attached to a plethysmograph. In both locations, the plethysmographs used were manufactured by the same company (Hokanson-Al-6, USA, in Brazil; EC6, Hokanson, Inc., Bellevue, WA, in Germany). The limb was positioned slightly elevated in relation to the heart to enable an adequate venous drainage. Two sphygmomanometer cuffs were place around the wrist and the arm, and the cuff placed on the wrist remained inflated to a suprasystolic pressure throughout the measurement while the upper cuff was inflated and deflated in a $20 \mathrm{~s}$ interval fashion characterized by $10 \mathrm{~s}$ in each phase.

\section{Laboratory measurements}

Blood samples were collected in the morning from an antecubital vein after an overnight fast. Laboratory tests included serum sodium $(\mathrm{mEq} / \mathrm{L})$, serum potassium $(\mathrm{mEq} / \mathrm{L})$, fasting glucose $(\mathrm{mg} / \mathrm{dL})$, creatinine $(\mathrm{mg} / \mathrm{dL})$, haemoglobin (g/dL), high-sensitivity C-reactive protein (hs-CRP; $\mathrm{mg} / \mathrm{L}$ ), and urea $(\mathrm{mg} / \mathrm{dL})$. B-type natriuretic peptide (BNP; pg/mL) plasma level was measured in Brazilian patients, and $\mathrm{N}$-terminal pro-B-type natriuretic peptide (NT-proBNP; ng/L) was measured in German patients.

\section{Statistical analysis}

Descriptive data are presented as mean \pm standard deviation, median with percentiles [95\% confidence interval $(\mathrm{Cl})$ ], and frequencies with percentages for categorical variables. One-sample Kolmogorov-Smirnov test was performed to assess the Gaussian distribution of the variables. Serum level of hs-CRP and FBF was not normally distributed, and, thus, Kruskal-Wallis test was used to compare the differences between the three groups (dilated cardiomyopathy, ischaemic, and chagasic patients). Analysis of variance with Scheffe's post hoc analysis was performed for all continuous and parametric variables, whereas the $\chi^{2}$ test was applied to compare categorical variables between groups. Pearson's correlation and logistic regression analyses were used as appropriate. A $P$-value lower than 0.05 was considered statistically significant in all statistical tests. For statistical analysis, the Statistical Package for the Social Sciences version 23 for Windows (SPSS Inc., Chicago, Illinois, USA) was used.

\section{Results}

A sample of 64 male patients with HF was analysed after matching for BMI and NYHA class, 20 of whom had dilated cardiomyopathy, 22 had ischaemic HF (both groups from SICA-HF), and 22 had Chagas disease (from TESTO-HF, Table 1). Fifteen patients were identified to have sarcopenia, of 
which five patients with dilated cardiomyopathy (ALM) height $^{2}=6.1 \pm 0.4 \mathrm{~kg} / \mathrm{m}^{2}$ ), five ischaemic patients (ALM) height $^{2}=6.3 \pm 0.4 \mathrm{~kg} / \mathrm{m}^{2}$ ), and five chagasic patients (ALM) height $\left.^{2}=6.3 \pm 0.6 \mathrm{~kg} / \mathrm{m}^{2}\right)$. There was no statistical difference in the distribution of sarcopenia between groups $(P=0.87)$.

The median value for BNP in patients with Chagas disease was $758 \mathrm{pg} / \mathrm{mL}(95 \% \mathrm{Cl}, 188-1007 \mathrm{pg} / \mathrm{mL})$. The median values for NT-proBNP in dilated cardiomyopathy and ischaemic patients were $976 \mathrm{ng} / \mathrm{L}(95 \% \mathrm{Cl}, 169-3217)$ and 1354 ng/L (95\% Cl, 880-3775), respectively. Baseline characteristics are shown in Table 1. Ischaemic patients were older than patients with Chagas disease $(67 \pm 9$ vs. $58 \pm 6$ years; $P=0.01$ ). Chagasic patients had lower height in comparison with patients with dilated cardiomyopathy and ischaemic $\mathrm{HF}$ (1.65 \pm 0.06 vs. $1.71 \pm 0.09$ vs. $1.75 \pm 0.07 \mathrm{~m}$, respectively; $P<0.05)$. Body weight was lower in patients with Chagas disease than in patients with dilated cardiomyopathy $(67.8 \pm 13.8$ vs. $84.9 \pm 18.5 \mathrm{~kg} ; P=0.003)$, whereas $\mathrm{BMI}$ was higher in patients with dilated cardiomyopathy compared with ischaemic and chagasic patients (28.8 \pm 5.1 vs.

Table 1 Baseline characteristics of all patients

\begin{tabular}{|c|c|c|c|}
\hline Variable & $\begin{array}{c}\text { Dilated } \\
\text { cardiomyopathy } \\
(n=20)\end{array}$ & $\begin{array}{l}\text { Ischaemic } \\
(n=22)\end{array}$ & $\begin{array}{l}\text { Chagasic } \\
(n=22)\end{array}$ \\
\hline Age (years) & $64 \pm 14$ & $67 \pm 9^{\dagger}$ & $58 \pm 6$ \\
\hline Weight (kg) & $84.9 \pm 18.5$ & \multirow{2}{*}{\multicolumn{2}{|c|}{$\begin{array}{l}77.6 \pm 13.267 .8 \pm 13.8 \\
1.75 \pm 0.07^{\dagger} 1.65 \pm 0.06\end{array}$}} \\
\hline Height $(m)$ & $1.71 \pm 0.09$ & & \\
\hline BMI $\left(\mathrm{kg} / \mathrm{m}^{2}\right)$ & $28.8 \pm 5.1$ & $25.4 \pm 3.9^{*}$ & $24.7 \pm 4.3^{*}$ \\
\hline NYHA (II/III) & $11 / 9$ & $13 / 9$ & $13 / 9$ \\
\hline LVEF (\%) & $31 \pm 7$ & $24 \pm 8^{*}$ & $28 \pm 7$ \\
\hline Heart rate (b.p.m.) & $66 \pm 11$ & $66 \pm 11$ & $69 \pm 9$ \\
\hline Sodium (mmol/L) & $141 \pm 4$ & $141 \pm 3$ & $139 \pm 4$ \\
\hline $\begin{array}{l}\text { Potassium (mmol/ } \\
\text { L) }\end{array}$ & $4.4 \pm 0.5$ & $4.5 \pm 0.5$ & $4.5 \pm 0.4$ \\
\hline Glucose (mg/dL) & $99 \pm 17$ & $110 \pm 48$ & $110 \pm 17$ \\
\hline Creatinine $(\mathrm{mg} / \mathrm{dL})$ & $1.26 \pm 0.58$ & $1.37 \pm 0.63$ & $1.39 \pm 0.45$ \\
\hline $\begin{array}{l}\text { Haemoglobin (g/ } \\
\mathrm{dL})\end{array}$ & $13.6 \pm 2.0$ & $13.3 \pm 1.7$ & $13.6 \pm 1.6$ \\
\hline $\mathrm{hs}-\mathrm{CRP}(\mathrm{mg} / \mathrm{L})$ & $3.85(1.06-7.0)$ & $\begin{array}{c}2.76(0.95- \\
3.41)\end{array}$ & $\begin{array}{c}3.25(1.59- \\
6.50)\end{array}$ \\
\hline $\begin{array}{l}\text { Urea }(\mathrm{mg} / \mathrm{dL}) \\
\text { Medication }\end{array}$ & $57 \pm 35$ & $59 \pm 22$ & $60 \pm 29$ \\
\hline Beta-blocker, $n(\%)$ & $20(100)$ & $21(95)$ & $22(100)$ \\
\hline ACE-I/ARB, $n(\%)$ & $19(95)$ & $20(91)$ & $22(100)$ \\
\hline Diuretics, $n(\%)$ & $18(90)$ & $17(77)$ & $19(86)$ \\
\hline Statins, $n(\%)$ & $10(50)$ & $22(100)^{\ddagger}$ & $10(45)$ \\
\hline MRA, $n(\%)$ & $12(60)$ & $13(59)$ & $15(68)$ \\
\hline Aspirin, $n(\%)$ & $6(30)$ & $19(86)^{\ddagger}$ & $3(14)$ \\
\hline $\begin{array}{l}\text { Calcium channel } \\
\text { blocker, } n(\%)\end{array}$ & $2(10)$ & $1(05)$ & $1(05)$ \\
\hline Metformin, $n(\%)$ & $4(20)$ & $1(05)$ & $1(05)$ \\
\hline
\end{tabular}

ACE-I, angiotensin-converting enzyme inhibitor; $A R B$, angiotensin receptor blocker; BMI, body mass index; hs-CRP, high-sensitivity C-reactive protein; LVEF, left ventricular ejection fraction; MRA, mineralocorticoid receptor antagonist; NYHA, New York Heart Association.

Data are presented as mean \pm SD, median (with lower and upper quartiles), or frequencies and percentages.

$" P<0.05$ vs. dilated cardiomyopathy.

${ }^{\dagger} P<0.05$ vs. chagasic.

${ }^{\ddagger} P<0.01$ vs. dilated cardiomyopathy and chagasic.
$25.4 \pm 3.9$ vs. $24.7 \pm 4.3 \mathrm{~kg} / \mathrm{m}^{2}$, respectively; $\left.P=0.049\right)$. LVEF was reduced in ischaemic patients in comparison with dilated cardiomyopathy patients $(24 \pm 8$ vs. $31 \pm 7 ; P=0.013)$, whereas there was no difference in LVEF between patients with Chagas disease and the other aetiologies. There was no difference with regard to NYHA class, resting heart rate, serum levels of sodium, potassium, glucose, creatinine, hsCRP, urea, or haemoglobin between the three groups (Table 1).

Not surprisingly, use of statins and aspirin was higher in ischaemic patients when compared with Chagas disease and dilated cardiomyopathy patients $(P=0.001$ and $P=0.0002$, respectively), while there was no difference in the use of other medications (Table 1).

\section{Body composition, functional capacity, and handgrip strength}

Body composition, functional capacity, and handgrip strength parameters are described in Table 2. Patients with dilated cardiomyopathy presented with higher fat mass in arms, legs, trunk, and total in comparison with those patients with ischaemic HF and Chagas disease (all $P<0.05$ ). Moreover, trunk lean mass was lower in patients with Chagas disease than in ischaemic HF patients $(23.9 \pm 3.9$ vs. $27.4 \pm 3.6 \mathrm{~kg}$; $P=0.046$ ), whereas total LBM showed a trend to be lower in chagasic patients when compared with ischaemic HF

Table 2 Body composition, functional capacity, and handgrip strength of all patients

\begin{tabular}{|c|c|c|c|}
\hline Variable & $\begin{array}{c}\text { Dilated } \\
\text { cardiomyopathy } \\
(n=20)\end{array}$ & $\begin{array}{l}\text { Ischaemic } \\
(n=22)\end{array}$ & $\begin{array}{l}\text { Chagasic } \\
(n=22)\end{array}$ \\
\hline \multicolumn{4}{|l|}{ Fat mass $(\mathrm{kg})$} \\
\hline Arms & $2.3 \pm 0.9$ & $1.6 \pm 0.7^{*}$ & $1.7 \pm 1.0^{*}$ \\
\hline Legs & $8.2 \pm 3.1$ & $5.5 \pm 2.2_{*}^{*}$ & $5.5 \pm 3.7_{*}^{*}$ \\
\hline Trunk & $16.3 \pm 6.5$ & $11.4 \pm 5.3^{*}$ & $9.2 \pm 5.8^{*}$ \\
\hline Total & $27.6 \pm 9.4$ & $19.3 \pm 8.0^{*}$ & $16.3 \pm 8.1^{*}$ \\
\hline \multicolumn{4}{|l|}{ Lean mass $(\mathrm{kg})$} \\
\hline Arms & $5.8 \pm 1.6$ & $5.9 \pm 1.0$ & $5.8 \pm 1.0$ \\
\hline Legs & $17.4 \pm 3.5$ & $16.8 \pm 2.6$ & $16.0 \pm 3.1$ \\
\hline Trunk & $26.9 \pm 6.5$ & $27.4 \pm 3.6^{\dagger}$ & $23.9 \pm 3.9$ \\
\hline Total & $54.0 \pm 11.6$ & $54.2 \pm 6.3^{(\dagger)}$ & $48.3 \pm 7.6$ \\
\hline \multicolumn{4}{|l|}{ Bone mass $(\mathrm{kg})$} \\
\hline Arms & $0.4 \pm 0.1$ & $0.4 \pm 0.1$ & $0.4 \pm 0.1$ \\
\hline Legs & $1.2 \pm 0.3^{\dagger}$ & $1.2 \pm 0.2^{\dagger}$ & $1.0 \pm 0.2$ \\
\hline Trunk & $0.9 \pm 0.2^{\dagger}$ & $0.9 \pm 0.2^{\dagger}$ & $0.7 \pm 0.2$ \\
\hline Total & $2.9 \pm 0.6^{(\dagger)}$ & $3.0 \pm 0.4^{\dagger}$ & $2.6 \pm 0.6$ \\
\hline $\begin{array}{l}\text { Peak } \\
\text { min) }\end{array}$ & $1.50 \pm 0.45$ & $1.15 \pm 0.36^{*}$ & ${ }^{*} 1.17 \pm 0.36^{*}$ \\
\hline $\begin{array}{l}\text { Peak } \mathrm{VO}_{2}(\mathrm{~mL} / \\
\mathrm{kg} / \mathrm{min})\end{array}$ & $17.5 \pm 4.5$ & $15.4 \pm 6.0$ & $17.5 \pm 4.7$ \\
\hline $\begin{array}{l}\text { Handgrip } \\
\text { strength (kg) }\end{array}$ & $36 \pm 14^{\dagger}$ & $37 \pm 11^{\dagger}$ & $27 \pm 8$ \\
\hline
\end{tabular}

$\mathrm{VO}_{2}$, oxygen consumption.

Data are presented as mean \pm SD.

" $P<0.05$ vs. dilated cardiomyopathy.

${ }^{\dagger} P<0.05$ vs. chagasic. Symbols in brackets denote a trend with $P<0.10$. 
patients ( $48.3 \pm 7.6$ vs. $54.2 \pm 6.3 \mathrm{~kg} ; P=0.09)$. In addition, bone mineral content in legs and trunk was lower in patients with Chagas disease compared with ischaemic $(P=0.02$ and $P=0.02$, respectively) and dilated cardiomyopathy patients ( $P=0.02$ and $P=0.04$, respectively). Total bone mineral content was also reduced in patients with Chagas disease in comparison with ischaemic HF patients ( $2.6 \pm 0.6 \mathrm{vs}$. $3.0 \pm 0.4 \mathrm{~kg}$; $P=0.045)$, and there was a tendency for a similar effect also when compared with dilated cardiomyopathy patients $(P=0.09)$.

Absolute peak $\mathrm{VO}_{2}$ was lower in chagasic and ischaemic $\mathrm{HF}$ patients when compared with patients with dilated cardiomyopathy ( $P=0.03$ and $P=0.02$, respectively), while there was no difference for relative peak $\mathrm{VO}_{2}$ among the three groups (Table 2). In addition, handgrip strength was reduced in patients with Chagas disease as compared with those with dilated cardiomyopathy $(P=0.03)$ and ischaemic heart disease $(P=0.02)$.

\section{Pearson's correlations and logistic regression}

Forearm blood flow was reduced in patients with Chagas disease when compared with patients with ischaemic $\mathrm{HF}$ and dilated cardiomyopathy [1.68 (95\% Cl, 1.37-2.11) vs. 2.75 (95\% $\mathrm{Cl}, 2.19-3.25)$ vs. $3.58(95 \% \mathrm{Cl}, 2.66-4.42) \mathrm{mL} / \mathrm{min} / 100 \mathrm{~mL}$, respectively; $P<0.01$; Figure 1]. On the other hand, there was a trend towards higher FBF in patients with dilated cardiomyopathy than in ischaemic HF patients $(P=0.056$; Figure 1). In addition, FBF was positively correlated with LBM $(r=0.31 ; P=0.012$; Figure $2 A)$, ALM $(r=0.25 ; P=0.046$; Figure $2 B)$, and handgrip strength ( $r=0.36 ; P=0.004$; Figure $2 C)$.

In a univariable logistic regression analysis using absolute peak $\mathrm{VO}_{2}$ (median value $>1.15 \mathrm{~L} / \mathrm{min}$ ) as the dependent variable, haemoglobin [odds ratio (OR), 1.598; 95\% Cl, 1.1242.273; $P=0.009$ ], ALM (OR, 1.222; 95\% Cl, 1.059-1.410; $P=0.006)$, and FBF $<2.61$ (OR, 0.360; 95\% Cl, 0.131-0.991;
$P=0.048)$ were associated with higher absolute peak $\mathrm{VO}_{2}$. Furthermore, in a multivariable logistic regression analysis using absolute peak $\mathrm{VO}_{2}$ (median value $>1.15 \mathrm{~L} / \mathrm{min}$ ) as the dependent variable, haemoglobin (OR, 1.506; $95 \% \mathrm{Cl}$, 1.043-2.177; $P=0.029$ ) and ALM (OR, 1.179; $95 \% \mathrm{Cl}$, 1.011-1.374; $P=0.035$ ) were independently associated with absolute peak $\mathrm{VO}_{2}$ adjusted for age, NYHA, creatinine, LVEF, and FBF (Table 3).

\section{Discussion}

Our study demonstrates a large overlap in clinical findings among patients with HF due to ischaemic heart disease, idiopathic dilated cardiomyopathy, and Chagas disease. Metabolic patterns, however, differ in patients with Chagas disease who present with lower LBM, bone mineral content, lower peak $\mathrm{VO}_{2}$, lower handgrip strength, and lower FBF. Predictors of peak $\mathrm{VO}_{2}$ include haemoglobin and ALM. The distribution of overt skeletal muscle wasting (sarcopenia) was similar in the three groups.

Chagas disease has been described as a public health issue in endemic countries in Latin America, and migration is thought to cause spreading of the disease outside of its original geographic boundaries leading to a global concern. ${ }^{18,19}$ The clinical features of patients with Chagas disease and HF resemble those of patients with ischaemic heart disease and idiopathic dilated cardiomyopathy, ${ }^{20}$ making the diagnosis and clinical management of these patients more complicated. ${ }^{21}$ In addition, patients with Chagas disease and HF have been commonly reported to be younger than their counterparts with HF from other aetiologies, ${ }^{5,22}$ and such difference in age was also found in our sample.

Additionally, our study is the first to show reductions in compartmental and total bone mineral content in patients with Chagas disease compared with dilated cardiomyopathy

FIGURE 1 Forearm blood flow (FBF) in dilated cardiomyopathy, ischaemic, and chagasic patients.

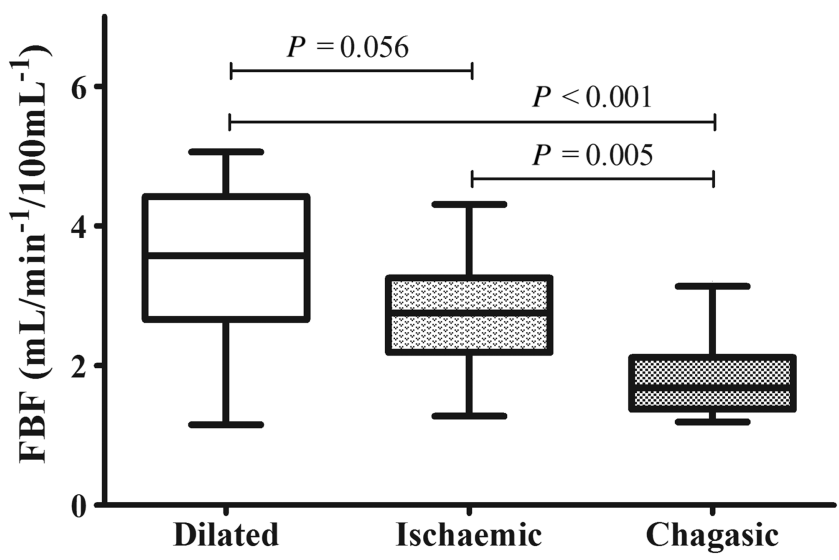


FIGURE 2 (A) Pearson's correlation with forearm blood flow (FBF) and lean body mass (LBM). (B) Pearson's correlation with FBF and appendicular lean mass (ALM). (C) Pearson's correlation with FBF and handgrip strength.
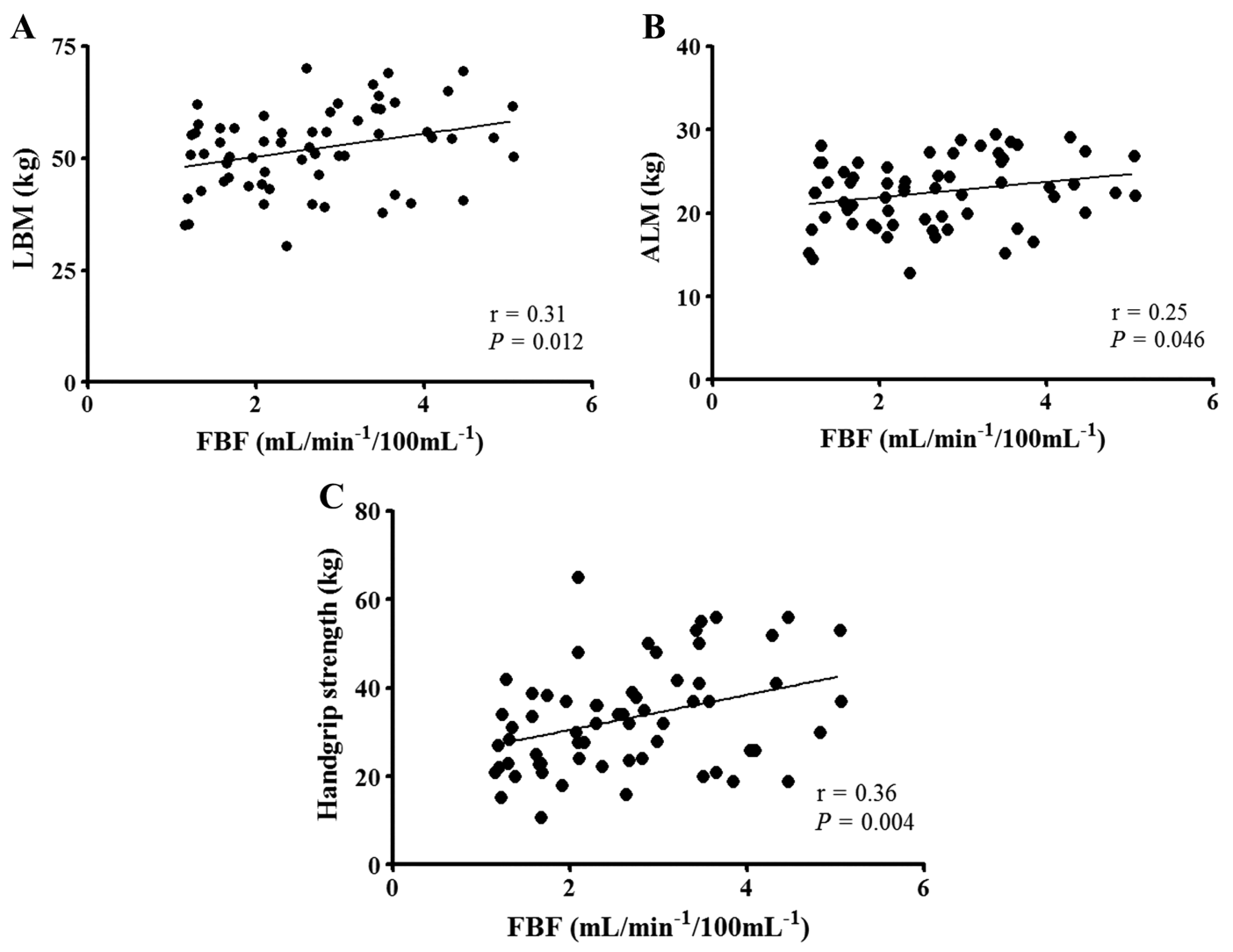

and ischaemic HF patients. Moreover, fat mass was reduced in chagasic and ischaemic patients, which may be a sign of an initial state of catabolism in these patients, because loss of fat mass preceding muscle wasting has been shown to be a cachexia phenotype in other populations. ${ }^{23}$

Although total LBM showed a tendency to be reduced in patients with Chagas disease compared with ischaemic HF patients, these results may be due to the small sample size in our study. Moreover, taking the mean age difference between these groups into consideration, we can also imply that reductions in total LBM may occur in these patients in
10 years of time, because of Chagas disease progression associated with HF and the aging process itself. Interestingly, in an experimental model of animals infected with $T$. cruzi, the presence of the parasite in the skeletal muscle produced acute and chronic mitochondrial dysfunction that led to permanent skeletal muscle alterations. ${ }^{24}$ In addition, chagasic patients with cardiomyopathy have shown to have an impairment in oxygen delivery due to abnormal muscle microvasculature. ${ }^{25}$

Reduced physical functioning and quality of life evaluated by questionnaires have been documented in patients with

Table 3 Logistic regression model using absolute peak oxygen consumption (median $>1.15 \mathrm{~L} / \mathrm{min}$ ) as the dependent variable

\begin{tabular}{lccccc}
\hline & \multicolumn{3}{c}{ Univariate analysis } & \multicolumn{2}{c}{ Multivariate analysis } \\
\cline { 2 - 6 } Variable & OR & $95 \% \mathrm{Cl}$ & $P$-value & OR & $95 \% \mathrm{Cl}$ \\
\hline Age (per year increase) & 0.976 & $0.931-1.023$ & 0.307 & & \\
NYHA (per class increase) & 2.493 & $0.899-6.916$ & 0.079 & & \\
Creatinine (per 1 mg/dL increase) & 0.710 & $0.284-1.770$ & 0.462 & & \\
Haemoglobin (per 1 g/dL increase) & 1.598 & $1.124-2.273$ & 0.009 & 1.506 & $1.043-2.177$ \\
LVEF (per 1\% increase) & 1.053 & $0.984-1.127$ & 0.136 & & 0.029 \\
ALM (per 500 g increase) & 1.222 & $1.059-1.410$ & 0.006 & 1.179 & $1.011-1.374$ \\
FBF $<2.61 \mathrm{~mL} / \mathrm{min} / 100 \mathrm{~mL}$ (present) & 0.360 & $0.131-0.991$ & 0.048 & 0.472 & $0.153-1.459$
\end{tabular}

ALM, appendicular lean mass; Cl, confidence interval; FBF, forearm blood flow; LVEF, left ventricular ejection fraction; NYHA, New York Heart Association; OR, odds ratio. 
Chagas disease and HF after only 1 year of follow-up, while pharmacological care improved parameters related to quality of life in these patients. ${ }^{26}$ In our sample, we found that handgrip strength, a predictor of worse outcomes in patients with $\mathrm{HF}^{27}$ was reduced in chagasic patients compared with both ischaemic and dilated cardiomyopathy patients. In addition, the impairment of muscle strength has been shown to affect not only appendicular skeletal muscle but also respiratory muscle in patients with Chagas disease and $\mathrm{HF}^{9}{ }^{9}$ suggesting that respiratory muscle weakness may influence ventilatory parameters contributing to reduce peak $\mathrm{VO}_{2}$ as we have demonstrated in chagasic patients in our study. ${ }^{28}$

Another factor that may lead to diminished functional capacity and reduced LBM is endothelial dysfunction, ${ }^{29}$ which was shown to be present in Chagas disease patients without $\mathrm{HF}^{30}$ and also in patients with $\mathrm{HF}$ regardless of aetiology. ${ }^{31}$ In our study, patients with Chagas disease and HF showed a reduction in FBF compared with the other two aetiologies (Figure 1), and this decrease in peripheral blood flow was positively correlated with muscle strength, LBM, and ALM (Figure 2).

Furthermore, sympathetic nerve activity, which also controls endothelial function, has been shown to be a hallmark in patients with $\mathrm{HF}$ and is also associated with impaired $\mathrm{VO}_{2}$ during exercise in these patients. ${ }^{32}$ Some studies have shown that patients with Chagas disease present with increased sympathetic outflow $^{33,34}$ just like patients with ischaemic HF compared with those with non-ischaemic disease. ${ }^{35}$ However, other groups have failed to find a difference in sympathetic activation across different aetiologies in patients with HF including Chagas disease. ${ }^{36,37}$ Taken together, the pronounced reduction in FBF seen in chagasic patients with $\mathrm{HF}$ in our study may be due to the combination of Chagas disease and HF with the involvement of peripheral mechanisms but may also be due to an interplay between sympathetic activation and the immune response of these patients. ${ }^{38}$

In addition, we found that haemoglobin and ALM were independent predictors of reduced absolute peak $\mathrm{VO}_{2}$. Regardless of HF aetiology, anaemia has been associated with worsening $\mathrm{HF}^{39}$ just like low ALM. ${ }^{40}$ In univariable and multivariable logistic regression analysis for each group separately, only ALM in patients with dilated cardiomyopathy was independently associated with absolute peak $\mathrm{VO}_{2}$ (median value), implying that the association seen in our results was driven by a higher ALM of these patients (Table 3).

Therapies used in the treatment of $\mathrm{HF}$ in patients with Chagas disease include standard HF medications, but also the anti-trypanosomal drugs benznidazole and nifurtimox. These drugs, however, seem to be of limited value in the indeterminate phase of the infection, because clinical trials with these pharmacological agents have shown limited tolerability without significant positive effects on clinical outcomes in patients with Chagas cardiomyopathy. ${ }^{41,42}$ In addition, administration of nifurtimox has been associated with side effects that might include anorexia, digestive disorders, and weight loss, but none of the chagasic patients in our study were or had taken any anti-trypanosomal medication for Chagas disease. Additionally, the statistical differences in statin and aspirin use found in our study seem to be founded in the aetiology of HF, once ischaemic patients are more likely to take medication that attenuates ischaemia by improving the progression of atherosclerosis.

Furthermore, exercise training is an important complementary therapy in the treatment of patients with HF so is it for patients with Chagas disease. Cardiovascular rehabilitation programmes have shown to improve cardiac function and respiratory muscle strength in patients with Chagas disease and HF. ${ }^{43}$ In addition, in an experimental study in rats that performed aerobic exercise training for 9 weeks before inoculation of $T$. cruzi, exercise training has shown to reduce exposure time to the parasite infection and attenuate cardiac parasitism by diminishing collagen aggregation in cardiac structures, while sedentary animals presented a more severe progression of Chagas disease. ${ }^{44}$ Moreover, exercise training seems to enhance the host defence and improve immunological and inflammatory markers that attenuate skeletal myositis in these patients. ${ }^{45}$

\section{Limitations}

We have to acknowledge that our study has limitations. First, only male patients with LVEF $\leq 40 \%$ were included, so these findings may be not applied to female patients and to patients with HF with preserved ejection fraction. Second, although we tried to homogenize the sample, the number of patients that were included in the matching analysis was limited, making the sample size small. Third, although both trials have been prospectively conducted, follow-up was not analysed in our study to determine if these body composition and muscle strength alterations would lead to worse outcomes in a long term. Finally, we cannot exclude that there may be differences in the correction of peak $\mathrm{VO}_{2}$ between cycle ergometer and treadmill.

\section{Conclusions}

Patients with Chagas disease and HF have decreased fat mass and exhibit reduced peripheral blood flow and impaired muscle strength compared with ischaemic HF patients. In addition, patients with Chagas disease and HF show a tendency to have greater reduction in total LBM, with ALM remaining an independent predictor of reduced functional capacity in these patients. The percentage of patients affected by sarcopenia was equal between groups. 


\section{Conflict of interest}

None declared.

\section{Funding}

This study was financed in part by the Coordenação de Aperfeiçoamento de Pessoal de Nível Superior - Brazil (CAPES) - Finance Code 001. G.W.P.D.F. was supported by Conselho Nacional de Desenvolvimento Científico e
Tecnológico (CNPq; 148758/2016-9); M.R.S. by Fundação de Amparo à Pesquisa do Estado de São Paulo (FAPESP; 2016/24306-0); and C.E.N. by CNPq (303573/2015-5) and FAPESP (2015/22814-5). S.v.H. has been a paid consultant and/or received fees for lectures from Bayer, Boehringer Ingelheim, BRAHMS/Thermo Fisher, Chugai Pharma, Grünenthal, Helsinn, Novartis, Pharmacosmos, Respicardia, Roche, Servier, and Vifor. His research lab is supported by the German Center for Cardiovascular Research (DZHK), the Innovative Medicines Initiative - Joint Undertaking (IMI-JU 115621), and Boehringer Ingelheim and Amgen.

\section{References}

1. Ziaeian B, Fonarow GC. Epidemiology and aetiology of heart failure. Nat Rev Cardiol 2016; 13: 368-378.

2. Ponikowski P, Voors AA, Anker SD, Bueno H, Cleland JG, Coats AJ, Falk V, González-Juanatey JR, Harjola VP, Jankowska EA, Jessup M, Linde C, Nihoyannopoulos P, Parissis JT, Pieske B, Riley JP, Rosano GM, Ruilope LM, Ruschitzka F, Rutten FH, van der Meer P, Members ATF, Reviewers D. 2016 ESC Guidelines for the diagnosis and treatment of acute and chronic heart failure: the Task Force for the diagnosis and treatment of acute and chronic heart failure of the European Society of Cardiology (ESC). Developed with the special contribution of the Heart Failure Association (HFA) of the ESC. Eur $J$ Heart Fail 2016; 18: 891-975.

3. Shen L, Ramires F, Martinez F, Bodanese LC, Echeverría LE, Gómez EA, Abraham WT, Dickstein K, Køber L, Packer M, Rouleau JL, Solomon SD, Swedberg K, Zile MR, Jhund PS, Gimpelewicz CR, McMurray JJV, Committees P-HaAIa. Contemporary characteristics and outcomes in chagasic heart failure compared with other nonischemic and ischemic cardiomyopathy. Circ Heart Fail 2017: 10, e004361.

4. Nunes MC, Dones W, Morillo CA, Encina JJ, Ribeiro AL, Cardiology CoCDotISo. Chagas disease: an overview of clinical and epidemiological aspects. J Am Coll Cardiol 2013; 62: 767-776.

5. Vilas Boas LG, Bestetti RB, Otaviano AP, Cardinalli-Neto A, Nogueira PR. Outcome of Chagas cardiomyopathy in comparison to ischemic cardiomyopathy. Int J Cardiol 2013; 167: 486-490.

6. Botoni FA, Poole-Wilson PA, Ribeiro AL, Okonko DO, Oliveira BM, Pinto AS, Teixeira MM, Teixeira AL, Reis AM, Dantas JB, Ferreira CS, Tavares WC, Rocha MO. A randomized trial of carvedilol after renin-angiotensin system inhibition in chronic Chagas cardiomyopathy. Am Heart J 2007; 153: 544 e1-8.

7. Issa VS, Amaral AF, Cruz FD, Ferreira SM, Guimarães GV, Chizzola PR, Souza GE, Bacal F, Bocchi EA. Beta-blocker therapy and mortality of patients with Chagas cardiomyopathy: a subanalysis of the REMADHE prospective trial. Circ Heart Fail 2010; 3: 82-88.

8. Sherbuk JE, Okamoto EE, Marks MA, Fortuny E, Clark EH, Galdos-Cardenas G, Vasquez-Villar A, Fernandez AB, Crawford TC, Do RQ, Flores-Franco JL, Colanzi R, Gilman RH, Bern C. Biomarkers and mortality in severe Chagas cardiomyopathy. Glob Heart 2015; 10: 173-180.

9. Vieira FC, de Melo Marinho P, Brandão DC, Barbosae e Silva O. Respiratory muscle strength, the six-minute walk test and quality of life in Chagas cardiomyopathy. Physiother Res Int 2014; 19: 8-15.

10. von Haehling S, Lainscak M, Doehner W, Ponikowski P, Rosano G, Jordan J, Rozentryt P, Rauchhaus M, Karpov R, Tkachuk V, Parfyonova Y, Zaritskey AY, Shlyakhto EV, Cleland JG, Anker SD. Diabetes mellitus, cachexia and obesity in heart failure: rationale and design of the Studies Investigating Co-morbidities Aggravating Heart Failure (SICA-HF). $J$ Cachexia Sarcopenia Muscle 2010; 1: 187-194.

11. Fonseca GWPD, dos Santos MR, de Souza FR, Takayama L, Rodrigues Pereira RM, Negrão CE, Alves MNN. Discriminating sarcopenia in overweight/obese male patients with heart failure: the influence of body mass index. ESC Heart Fail 2020; 7: 84-91.

12. Cruz-Jentoft AJ, Bahat G, Bauer J, Boirie $\mathrm{Y}$, Bruyère $\mathrm{O}$, Cederholm $\mathrm{T}$, Cooper $\mathrm{C}$, Landi F, Rolland Y, Sayer AA, Schneider SM, Sieber CC, Topinkova E, Vandewoude M, Visser M, Zamboni M, Writing Group for the European Working Group on Sarcopenia in Older
People 2 (EWGSOP2) atEGfE. Sarcopenia: revised European consensus on definition and diagnosis. Age Ageing 2019; 48: 16-31.

13. Page E, Cohen-Solal A, Jondeau G, Douard H, Roul G, Kantelip JP, Bussière JL. Comparison of treadmill and bicycle exercise in patients with chronic heart failure. Chest 1994; 106: 1002-1006.

14. Kim S, Yamabe H, Yokoyama M. Hemodynamic characteristics during treadmill and bicycle exercise in chronic heart failure: mechanism for different responses of peak oxygen uptake. Jpn Circ J 1999; 63: 965-970.

15. Beckers PJ, Possemiers NM, van Craenenbroeck EM, van Berendoncks AM, Wuyts K, Vrints CJ, Conraads VM. Impact of exercise testing mode on exercise parameters in patients with chronic heart failure. Eur J Prev Cardiol 2012; 19: 389-395.

16. Maeder MT, Wolber T, Ammann P, Myers J, Brunner-La Rocca HP, Hack D, Riesen W, Rickli H. Cardiopulmonary exercise testing in mild heart failure: impact of the mode of exercise on established prognostic predictors. Cardiology 2008; 110: 135-141.

17. Witte KK, Clark AL. Cycle exercise causes a lower ventilatory response to exercise in chronic heart failure. Heart 2005; 91: 225-226.

18. Pérez-Molina JA, Molina I. Chagas disease. Lancet 2018; 391: 82-94.

19. Traina M, Meymandi S, Bradfield JS. Heart failure secondary to Chagas disease: an emerging problem in non-endemic areas. Curr Heart Fail Rep 2016; 13: 295-301.

20. Martinez F, Perna E, Perrone SV, Liprandi AS. Chagas disease and heart failure: an expanding issue worldwide. Eur Cardiol 2019; 14: 82-88.

21. Terhoch CB, Moreira HF, Ayub-Ferreira SM, Conceição-Souza GE, Salemi VMC, Chizzola PR, Oliveira MT, Lage SHG, Bocchi EA, Issa VS. Clinical findings 
and prognosis of patients hospitalized for acute decompensated heart failure: analysis of the influence of Chagas etiology and ventricular function. PLoS Negl Trop Dis 2018; 12: e0006207.

22. Braga AM, Rondon MU, Negrão CE, Wajngarten M. Predictive value of ventilatory and metabolic variables for risk of death in patients with cardiac failure. Arq Bras Cardiol 2006; 86: 451-458.

23. Kays JK, Shahda S, Stanley M, Bell TM, O'Neill BH, Kohli MD, Couch ME, Koniaris LG, Zimmers TA. Three cachexia phenotypes and the impact of fat-only loss on survival in FOLFIRINOX therapy for pancreatic cancer. $J \mathrm{Ca}$ chexia Sarcopenia Muscle 2018; 9: 673-684.

24. Báez AL, Reynoso MN, Lo Presti MS, Bazán PC, Strauss M, Miler N, Pons P, Rivarola HW, Paglini-Oliva P. Mitochondrial dysfunction in skeletal muscle during experimental Chagas disease. Exp Mol Pathol 2015; 98: 467-475.

25. Montes De Oca M, Torres SH, Loyo JG, Vazquez F, Hernández N, Anchustegui B, Puigbó JJ. Exercise performance and skeletal muscles in patients with advanced Chagas disease. Chest 2004; 125: 1306-1314

26. Chambela MDC, Mediano MFF, Carneiro FM, Ferreira RR, Waghabi MC, Mendes VG, Oliveira LS, de Holanda MT, de Sousa AS, da Costa AR, Xavier SS, da Silva GMS, Saraiva RM. Impact of pharmaceutical care on the quality of life of patients with heart failure due to chronic Chagas disease: randomized clinical trial. $\mathrm{Br} J$ Clin Pharmacol 2020; 86: 143-154.

27. Chung CJ, Wu C, Jones M, Kato TS, Dam TT, Givens RC, Templeton DL, Maurer MS, Naka Y, Takayama H, Mancini DM, Schulze PC. Reduced handgrip strength as a marker of frailty predicts clinical outcomes in patients with heart failure undergoing ventricular assist device placement. $J$ Card Fail 2014; 20: 310-315.

28. Habedank D, Meyer FJ, Hetzer R, Anker SD, Ewert R. Relation of respiratory muscle strength, cachexia and survival in severe chronic heart failure. $J \mathrm{Ca}$ chexia Sarcopenia Muscle 2013; 4: 277-285.

29. Dos Santos MR, Saitoh M, Ebner N, Valentova M, Konishi M, Ishida J,
Emami A, Springer J, Sandek A, Doehner W, Anker SD, von Haehling S. Sarcopenia and endothelial function in patients with chronic heart failure: results from the Studies Investigating Comorbidities Aggravating Heart Failure (SICA-HF). J Am Med Dir Assoc 2017; 18: $240-245$.

30. Plentz RD, Irigoyen MC, Muller AS, Casarini DE, Rubira MC, Moreno Junior $\mathrm{H}$, Mady C, Ianni BM, Krieger EM, Consolim-Colombo F. Venous endothelial dysfunction in Chagas' disease patients without heart failure. Arq Bras Cardiol 2006; 86: 466-471.

31. Zuchi C, Tritto I, Carluccio E, Mattei C, Cattadori G, Ambrosio G. Role of endothelial dysfunction in heart failure. Heart Fail Rev 2020; 25: 21-30.

32. Alves MJ, Rondon MU, Santos AC, Dias RG, Barretto AC, Krieger EM, Middlekauff HR, Negrão CE. Sympathetic nerve activity restrains reflex vasodilatation in heart failure. Clin Auton Res 2007; 17: 364-369.

33. da Cunha AB. Chagas' disease and the involvement of the autonomic nervous system. Rev Port Cardiol 2003; 22: 813-824.

34. Machado CR, Camargos ER, Guerra LB, Moreira MC. Cardiac autonomic denervation in congestive heart failure: comparison of Chagas' heart disease with other dilated cardiomyopathy. Hum Pathol 2000; 31: 3-10.

35. Notarius CF, Spaak J, Morris BL, Floras JS. Comparison of muscle sympathetic activity in ischemic and nonischemic heart failure. $J$ Card Fail 2007; 13: 470-475.

36. Negrão CE, Santos AC, Rondon MU, Franco FG, Ianni B, Rochitte CE, Braga AM, Oliveira MT, Mady C, Barretto AC, Middlekauff HR. Muscle sympathetic nerve activity in patients with Chagas' disease. Int $J$ Cardiol 2009; 137: 252-259.

37. Grassi G, Seravalle G, Bertinieri G, Turri C, Stella ML, Scopelliti F, Mancia G. Sympathetic and reflex abnormalities in heart failure secondary to ischaemic or idiopathic dilated cardiomyopathy. Clin Sci (Lond) 2001; 101: 141-146.

38. Roggero E, Pérez AR, Pollachini N, Villar SR, Wildmann J, Besedovsky H, Del Rey A. The sympathetic nervous system affects the susceptibility and course of
Trypanosoma cruzi infection. Brain Behav Immun 2016; 58: 228-236.

39. van der Wal $\mathrm{HH}$, Grote Beverborg N, Dickstein K, Anker SD, Lang CC, Ng LL, van Veldhuisen DJ, Voors AA, van der Meer P. Iron deficiency in worsening heart failure is associated with reduced estimated protein intake, fluid retention, inflammation, and antiplatelet use. Eur Heart $J$ 2019; 40: 3616-3625.

40. Fulster S, Tacke M, Sandek A, Ebner N, Tschope C, Doehner W, Anker SD, von Haehling S. Muscle wasting in patients with chronic heart failure: results from the Studies Investigating Co-morbidities Aggravating Heart Failure (SICA-HF). Eur Heart $J$ 2013; 34: 512-519.

41. Jackson Y, Alirol E, Getaz L, Wolff $\mathrm{H}$, Combescure C, Chappuis F. Tolerance and safety of nifurtimox in patients with chronic Chagas disease. Clin Infect Dis 2010; 51: e69-e75.

42. Morillo CA, Marin-Neto JA, Avezum A, Sosa-Estani S, Rassi A, Rosas F, Villena E, Quiroz R, Bonilla R, Britto C, Guhl F, Velazquez E, Bonilla L, Meeks B, Rao-Melacini P, Pogue J, Mattos A, Lazdins J, Connolly SJ, Yusuf S, Investigators B. Randomized trial of benznidazole for chronic Chagas' cardiomyopathy. $N$ Engl $J$ Med 2015; 373: 1295-1306.

43. Mediano MF, Mendes FS, Pinto VL, Silva GM, Silva PS, Carneiro FM, Sangenis LH, Saraiva RM, Xavier SS, Brasil PE, Hasslocher-Moreno AM, Sousa AS. Cardiac rehabilitation program in patients with Chagas heart failure: a single-arm pilot study. Rev Soc Bras Med Trop 2016; 49: 319-328.

44. Novaes RD, Gonçalves RV, Penitente AR, Bozi LH, Neves CA, Maldonado IR, Natali AJ, Talvani A. Modulation of inflammatory and oxidative status by exercise attenuates cardiac morphofunctional remodeling in experimental Chagas cardiomyopathy. Life Sci 2016; 152: 210-219.

45. Novaes RD, Gonçalves RV, Penitente AR, Cupertino MC, Maldonado IRSC, Talvani A, Natali AJ. Parasite control and skeletal myositis in Trypanosoma cruzi-infected and exercised rats. Acta Trop 2017; 170: 8-15. 